\title{
DAILY PHYSICAL FUNCTION AND FRAILTY IN PERSONS WITH PARKINSON'S DISEASE: A FOCUS ON FEMALES
}

\author{
by \\ Kaitlyn Patricia Roland \\ B.HSc., The University of Western Ontario, 2006 \\ M.Sc., The University of Western Ontario, 2008
}

\begin{abstract}
A THESIS SUBMITTED IN PARTIAL FULFILLMENT OF THE REQUIREMENTS FOR THE DEGREE OF
\end{abstract}

DOCTOR OF PHILOSOPHY

in

THE COLLEGE OF GRADUATE STUDIES

(Interdisciplinary Studies)

[Health and Exercise Sciences]

THE UNIVERSITY OF BRITISH COLUMBIA

(Okanagan)

September 2012

(C) Kaitlyn Patricia Roland, 2012 


\section{Abstract}

Persons with Parkinson's disease (PD) are often excluded from frailty studies, and thus little is known about how frailty influences decline in physiological capacity in persons with PD. Impaired physiological capacity impacts the ability to remain physically active, which reduces physical function necessary for independent living. The overall purpose of this dissertation was to examine physiological capacity (i.e., muscle activation patterns) and physical activity in males and females with PD during routine daily activities to determine whether they influence physical function and frailty. An extensive literature review of sex differences in PD highlights that greater declines in gait speed, balance and motor function occur in females compared with males. This dissertation demonstrated persons with PD and females were weaker and have less muscle quiescence, as measured with gaps in the electromyography (EMG), compared with controls and males with PD. These results provide insight into mechanisms (i.e., physiological capacity) that determine PD and sex-related declines in functional performance. In addition, greater muscle activity and less quiescence in females with PD may perpetuate frailty through increased muscle fatigue and slowness of movement. Females with PD are more vulnerable to prefrailty than males and factors that are associated with frailty are quality of life (QoL) and selfreported exhaustion. The neuromuscular changes associated with frailty exacerbate PD, which may create greater muscle fatigue resulting in self-reported exhaustion. In conclusion, research presented within this dissertation demonstrates that addressing frailty in PD is important, especially in females who are at greater risk for functional decline. This research presents new knowledge by suggesting frail females with PD remain physically active during daily life and that disease management may better reflect frailty than disease severity or duration. Understanding how frailty concurrently exists with PD and how these conditions progress within the aging adult may enhance identification and implementation of management strategies aimed at improving functional independence and QoL. 


\section{Preface}

This research project (H10-00149) was approved by the University of British Columbia Clinical Research Ethics Board on March $4^{\text {th }}, 2010$

Four manuscripts (Chapters 2, 5, 6, 7) are published in notable scientific journals.

- Roland, K.P., Jakobi, J.M. Powell, C., \& Jones, G. R. (2011). Factors related to functional independence in females with Parkinson's disease: a systematic review. Maturitas, 69(4), 304311.

I performed the systematic review and analysis. I wrote the first draft of the manuscript and was involved in the final editing.

• Roland, K.P., Cornett, K.M.D., Theou, O., Jakobi, J.M., \& Jones, G.R. (2012). Daily physical activity across frailty phenotypes in community-dwelling females with and without Parkinson’s disease. Journal of Aging Research, vol. 2012, Article ID 468156, 8 pages, 2012. doi:10.1155/2012/468156.

I recruited the subjects, executed the data collection and completed the analysis. I wrote the first draft of the manuscript and was involved in the final editing.

- Roland, K.P., Jakobi, J.M., Powell, C., \& Jones, G.R. (2012). Quality of life as a determinant of frailty phenotype in community-dwelling persons with Parkinson's disease. Journal of the American Geriatrics Society, 60(3), 590-592.

I recruited the subjects, executed the data collection and completed the analysis. I wrote the first draft of the manuscript and was involved in the final editing. 
- Roland, K.P., Cornett, K.M.D., Theou, O., Jakobi, J.M., \& Jones, G.R. (2012). Concurrence of frailty and Parkinson’s disease. The Journal of Frailty and Aging, 1(3), 123-127.

I recruited the subjects, executed the data collection and completed the analysis. I wrote the first draft of the manuscript and was involved in the final editing.

Two manuscripts are submitted for peer-review in notable scientific journals (Chapters 3, 4).

• Roland, K.P., Jones, G.R., Jakobi, J.M. Parkinson’s disease and sex differences in electromyography during daily life. Journal of Electromyography and Kinesiology. I recruited the subjects, executed the data collection and completed the analysis. I wrote the first draft of the manuscript and was involved in the final editing.

- Roland, K.P., Jones, G.R., Jakobi, J.M. Daily electromyography in females with Parkinson’s disease: A potential indicator of frailty? Aging, Clinical and Experimental Research. I recruited the subjects, executed the data collection and completed the analysis. I wrote the first draft of the manuscript and was involved in the final editing. 
Table of Contents

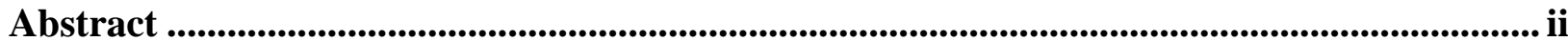

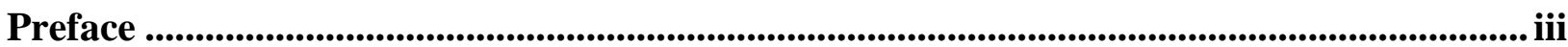

Table of Contents......................................................................................................................................... $\mathbf{v}$

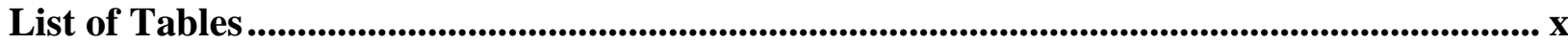

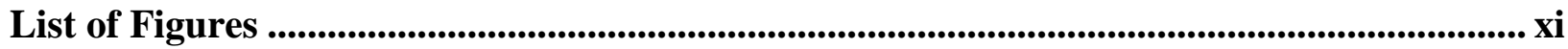

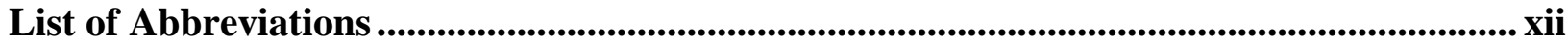

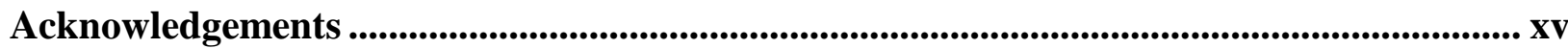

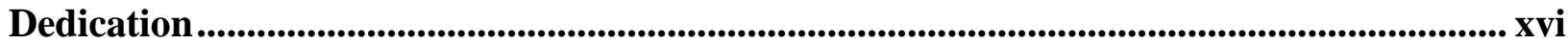

Chapter 1: Introduction and Background ................................................................................. 1

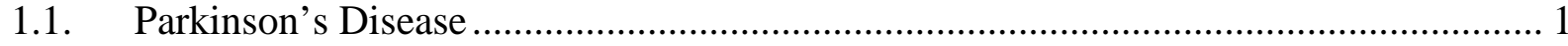

1.1.1. Disease Onset and Symptom Presentation .......................................................... 1

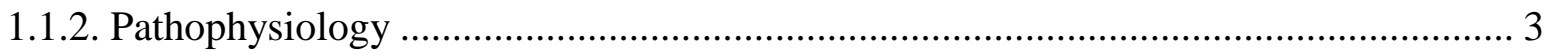

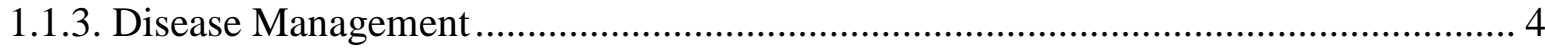

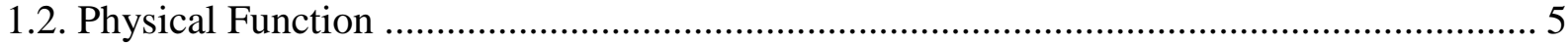

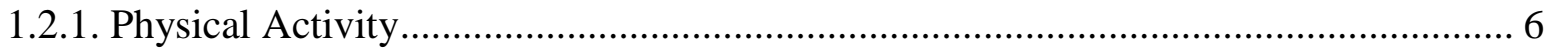

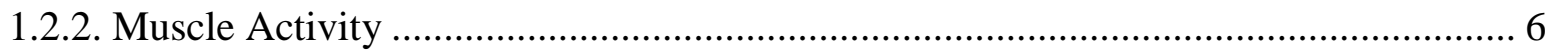

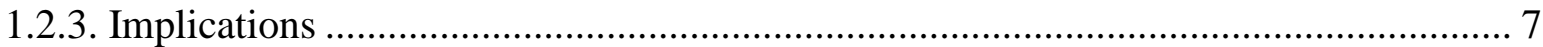

1.3. Sex Differences in Parkinson's Disease ...................................................................... 8

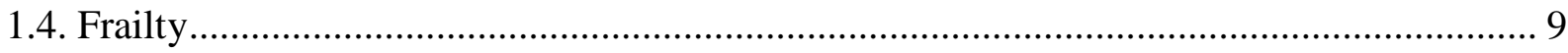

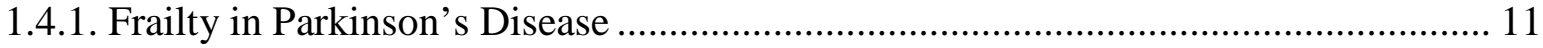

1.5. Research Design and Objectives of the Dissertation................................................... 12

1.5.1. Setting and Recruitment: Measurement of Independent Daily Activities ................. 14

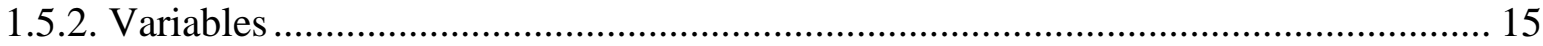

1.5.3. Objectives and Structure of the Dissertation ........................................................ 16

\section{Chapter 2: Factors Related to Functional Independence in Females with Parkinson's}

Disease: A Systematic Review.................................................................................................................. 18

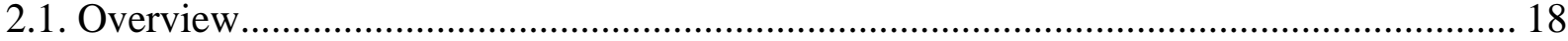

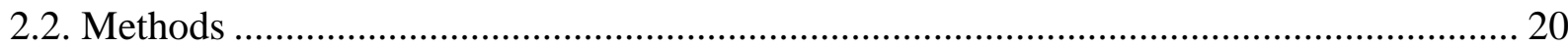

2.2.1. Literature Search Strategy .............................................................................. 20 
2.2.2. Data Extraction and Quality Analysis ......................................................... 21

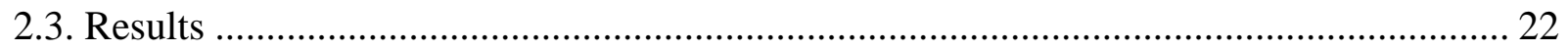

2.3.1. Literature Search........................................................................................... 22

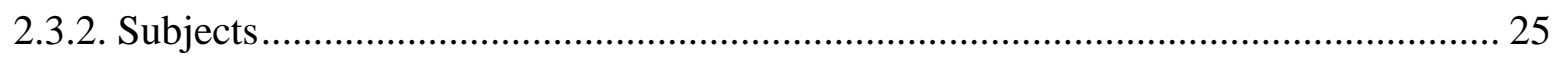

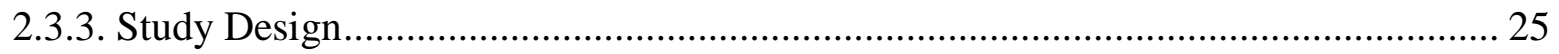

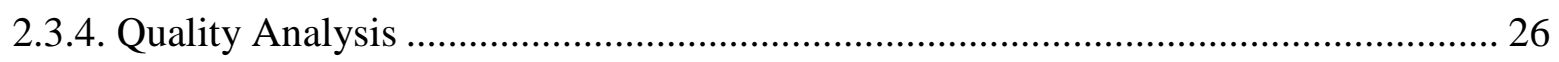

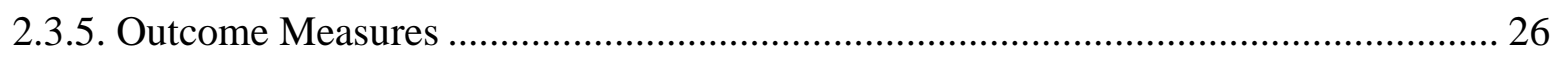

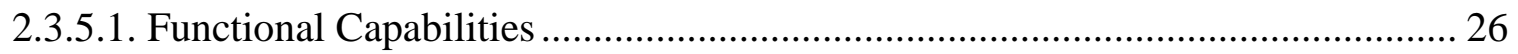

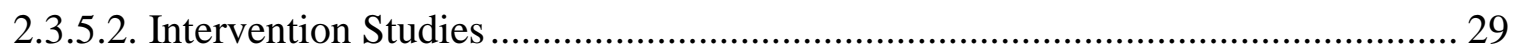

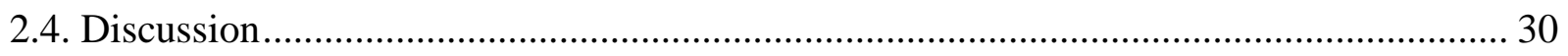

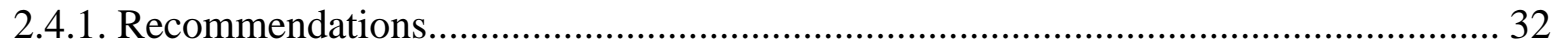

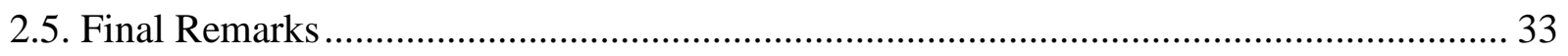

\section{Chapter 3: Parkinson's Disease and Sex Differences in Electromyography During Daily}

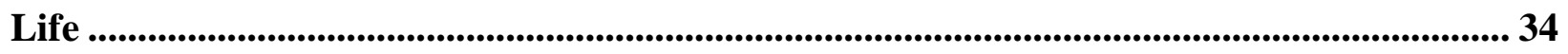

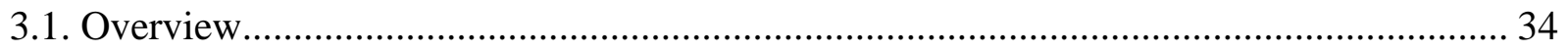

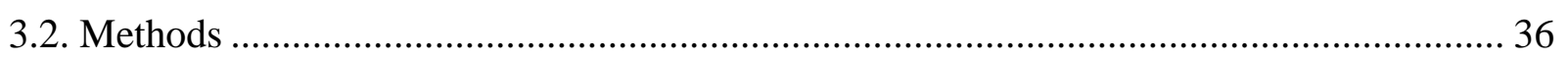

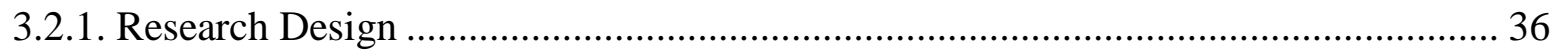

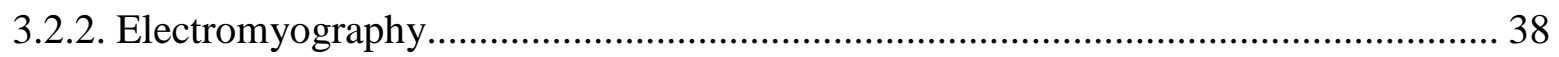

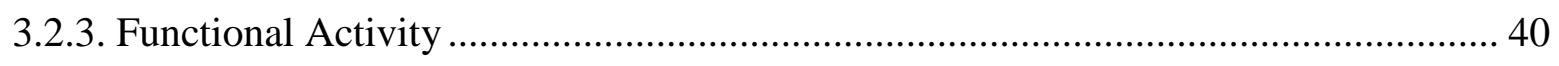

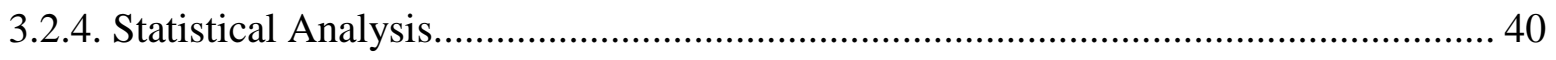

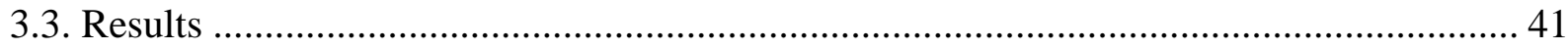

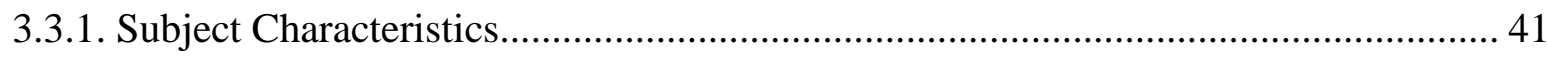

3.3.2. Daily Muscle Quiescence .......................................................................... 42

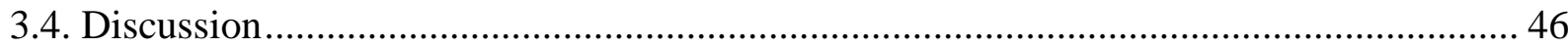

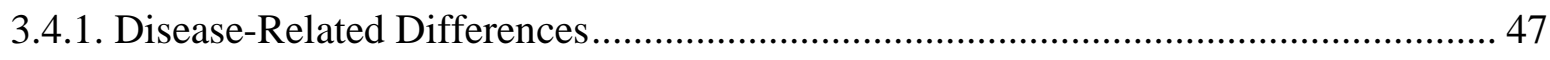

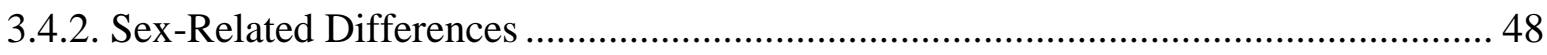

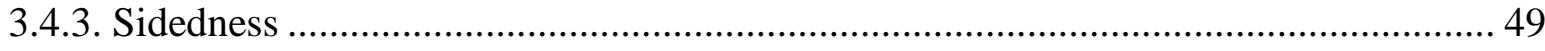

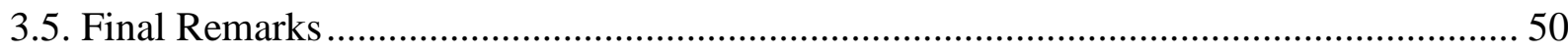

\section{Chapter 4: Daily Electromyography in Females with Parkinson's Disease: A Potential}

Indicator of Frailty? ................................................................................................................................ 51

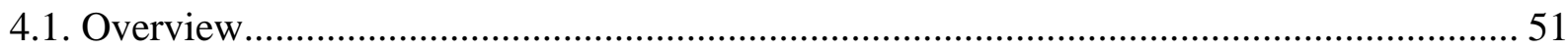

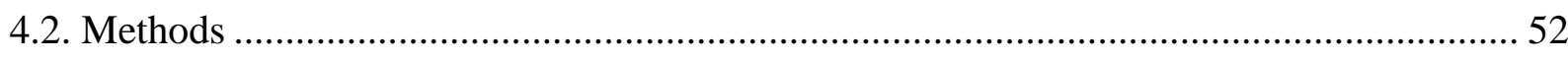




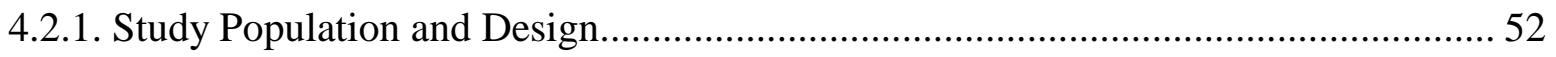

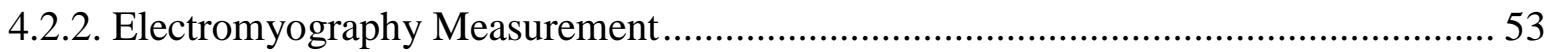

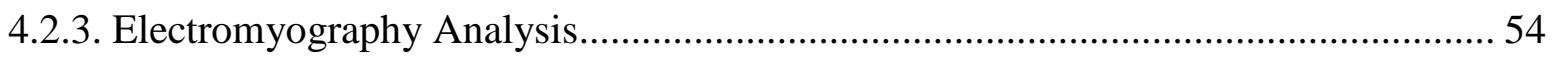

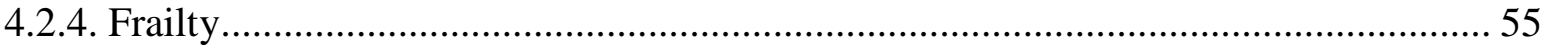

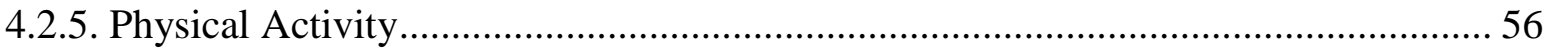

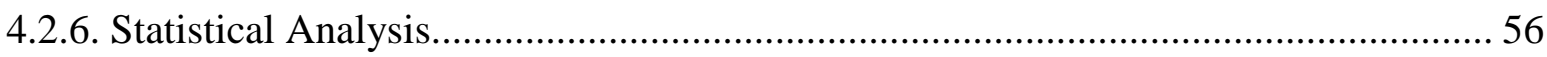

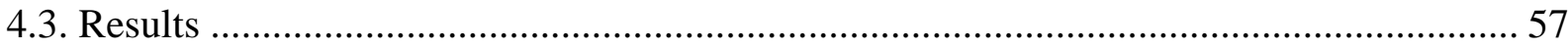

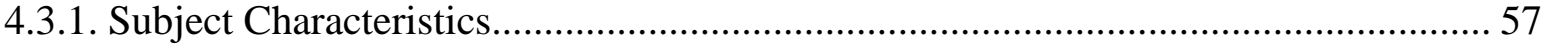

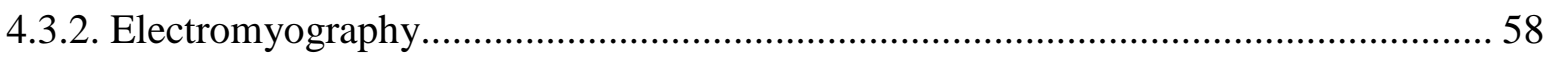

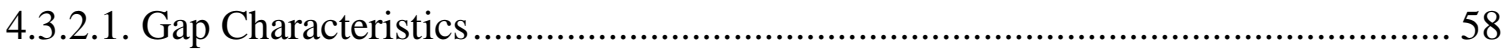

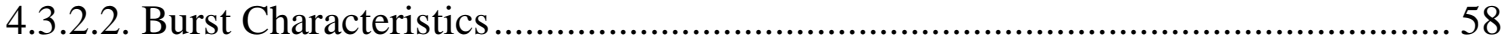

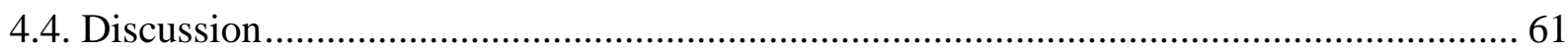

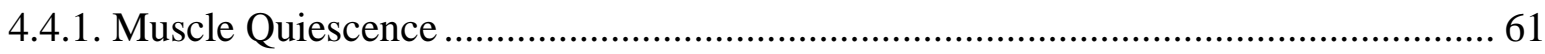

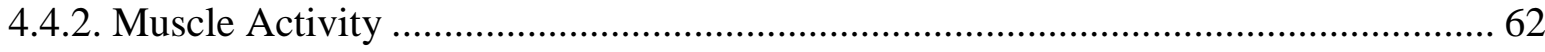

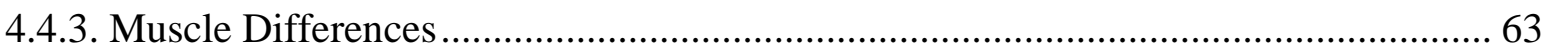

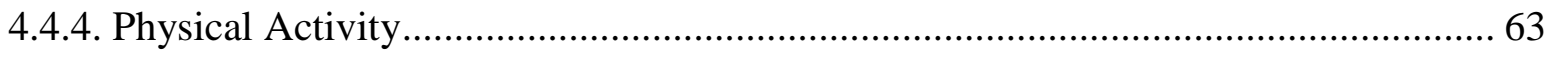

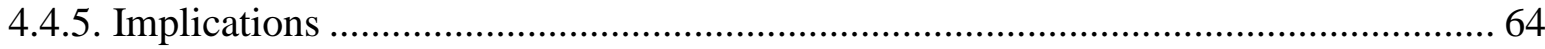

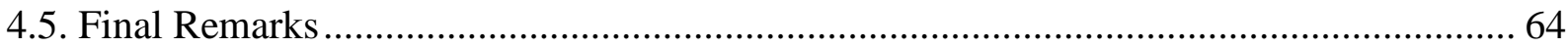

\section{Chapter 5: Physical Activity Across Frailty Phenotypes in Females with Parkinson's}

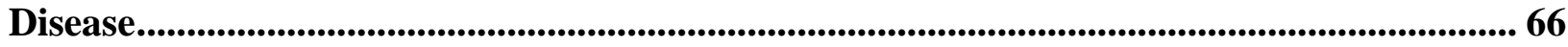

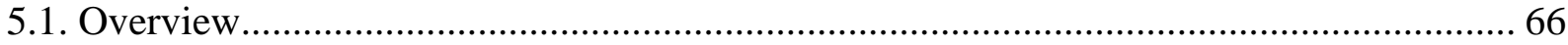

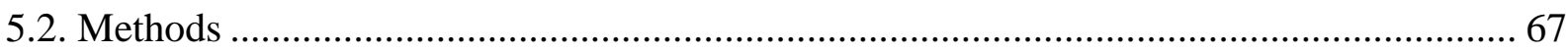

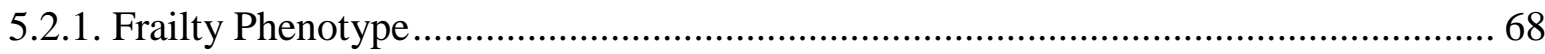

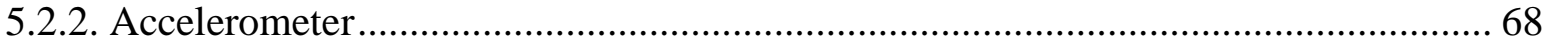

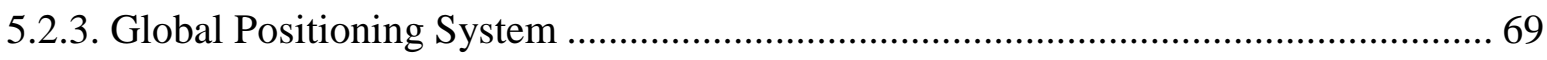

5.2.4. Self-Reported Physical Activities...................................................................... 69

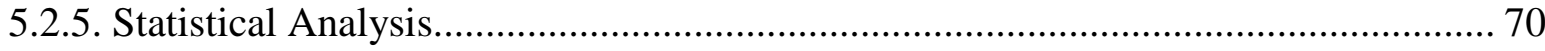

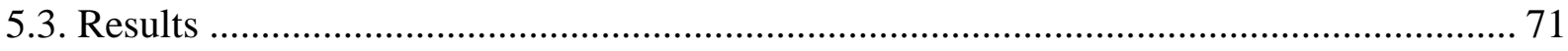

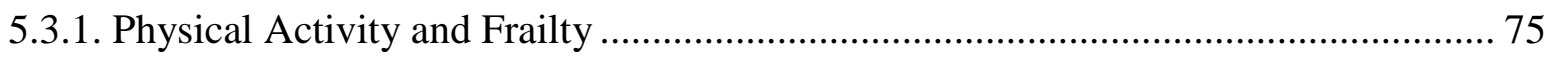

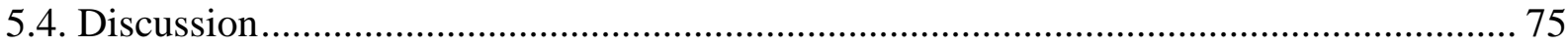

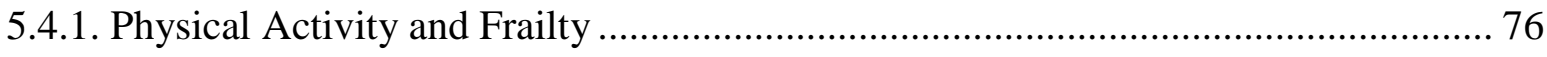

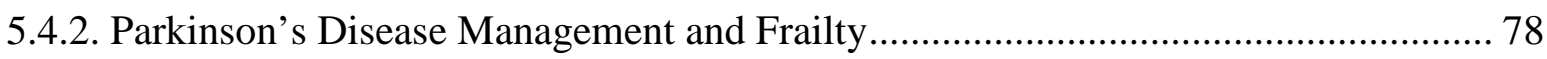




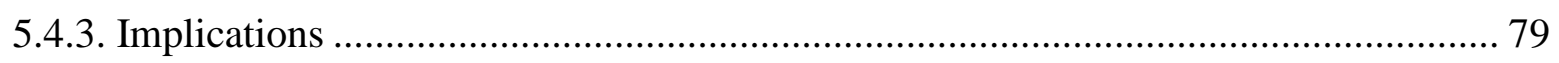

5.4.3.1. Independent-Living Populations and Neurological Disorders ............................. 79

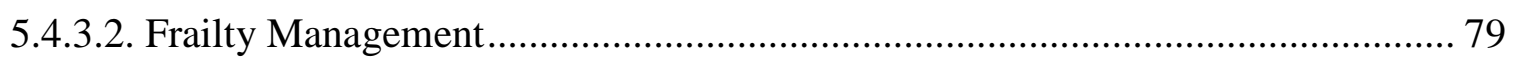

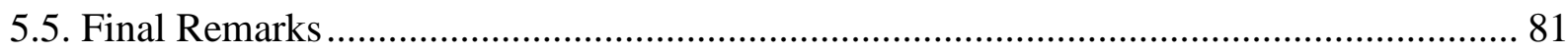

\section{Chapter 6: Quality of Life as a Determinant of Frailty Phenotype in Persons with}

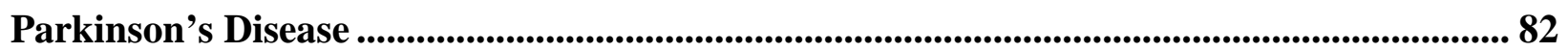

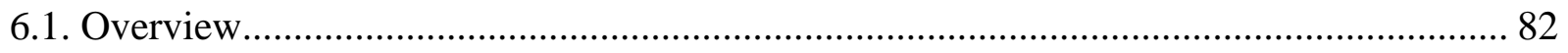

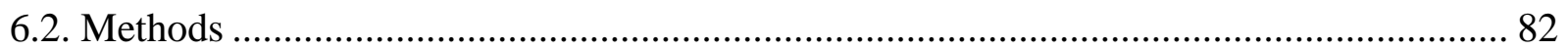

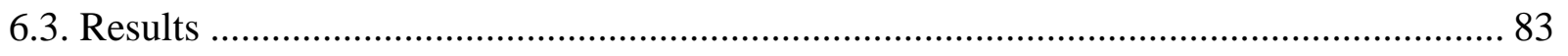

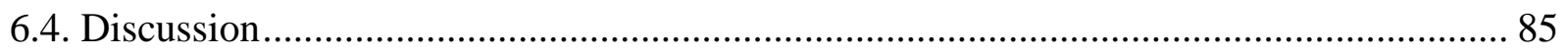

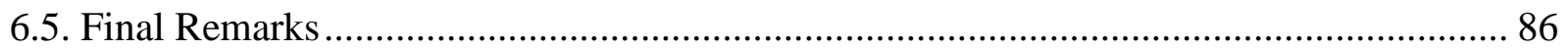

Chapter 7: Concurrence of Frailty and Parkinson's Disease ....................................................... 87

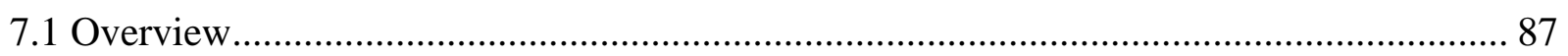

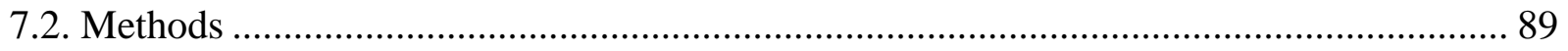

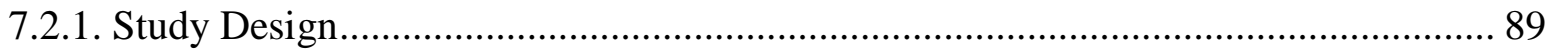

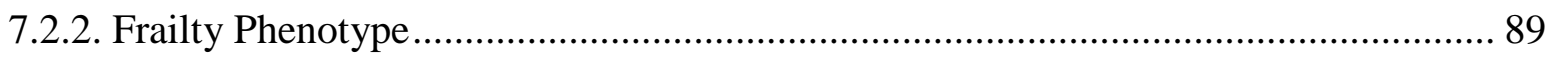

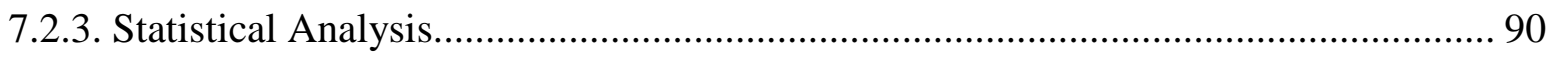

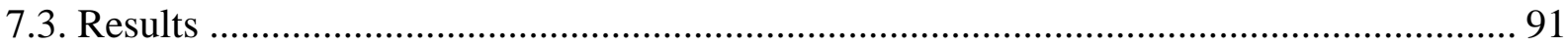

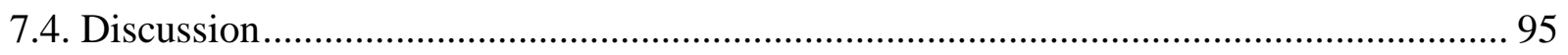

7.4.1. Carbidopa-Levodopa Use Indicates Frailty ………………......................................... 95

7.4.2. Age and Frailty ……....................................................................................................... 96

7.4.3. Is Comorbidity Masked by Parkinson’s Disease? ........................................................ 96

7.4.4. Frailty and Disease: Overlapping Concepts and Measures?........................................ 97

7.5. Final Remarks ................................................................................................................... 99

Chapter 8: Discussion and Conclusions................................................................................................. 100

8.1. Novel Contributions to Scientific Knowledge.................................................................. 100

8.1.1. Females with Parkinson’s Disease Experience Greater Functional Decline ............. 100

8.1.2. Daily Muscle Quiescence and Strength Differs in Parkinson's Disease ................... 101

8.1.3. Muscle Activation Differs with Frailty in Females with Parkinson's Disease.......... 102

8.1.4. Physical Activity Participation and Frailty in Females with Parkinson’s Disease ... 102

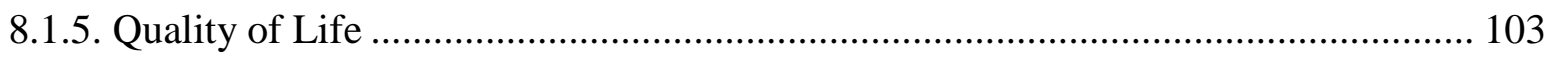

8.1.6. Parkinson’s Disease Symptom Management and Frailty ......................................... 103 


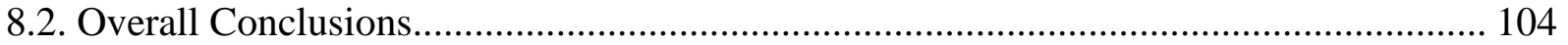

8.3. Limitations and Future Research Directions .................................................................... 106

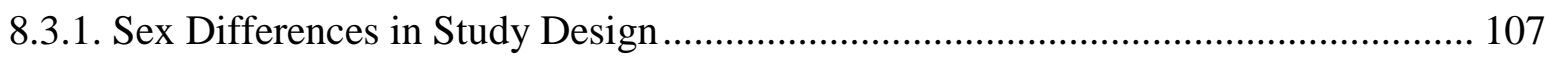

8.3.2. Muscle Activation Patterns in Parkinson’s Disease ................................................. 108

8.3.3. Frailty Assessment in Parkinson’s Disease ................................................................ 109

8.3.4. Causal Factors Related to Frailty in Parkinson's Disease ............................................ 110

8.3.5. Exercise and Rehabilitation Guidelines..................................................................... 110

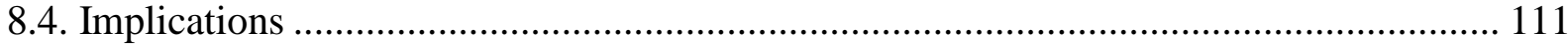

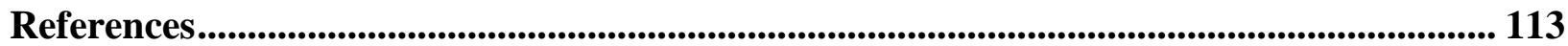

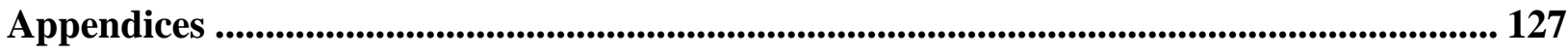

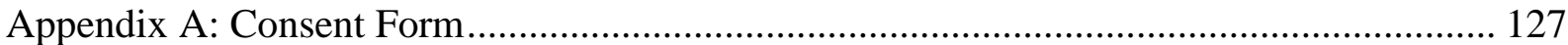

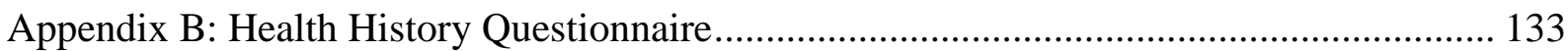

Appendix C: Minnesota Leisure Time Physical Activity Questionnaire (MLTA) ................ 135

Appendix D: Parkinson’s Disease Questionnaire-39 ............................................................. 136

Appendix E: Copyright Permissions for Reproduction of Journal Articles ............................ 139 


\section{List of Tables}

Table 2.1. Presentation of Parkinson's Disease in Females as Compared with Males ............... 20

Table 2.2. Results of the Systematic Literature Review ...................................................... 23

Table 3.1. Characteristics of Males and Females with Parkinson’s Disease and Controls .......... 42

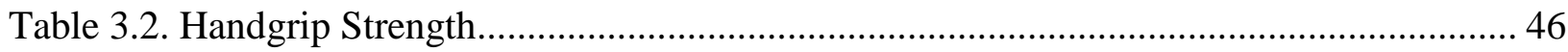

Table 4.1. Characteristics of Females Across Frailty Phenotypes......................................... 57

Table 5.1. Subject Characteristics Across Frailty Phenotypes …............................................ 71

Table 5.2. Main Effects on Physical Activity Variables .................................................... 72

Table 5.3. The Association of Physical Activity Variables to Frailty in Control Females ......... 73

Table 7.1. Subject Characteristics Across Frailty Phenotypes …............................................. 92

Table 7.2. Frailty Severity and Age- and Disease-Related Characteristics .............................. 93 


\section{List of Figures}

Figure 1.1. The Hoehn \& Yahr Scale ............................................................................. 3

Figure 1.2. Accumulation of Signs and Symptoms Associated with Stages of Frailty.............. 11

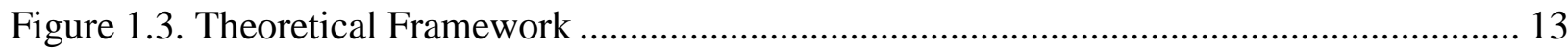

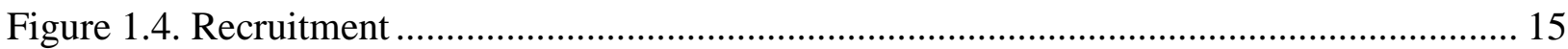

Figure 2.1. Flow Diagram of Study Inclusion According to PRISMA Statement ..................... 22

Figure 3.1. A Representative Electromyography Recording of Vastus Lateralis........................ 39

Figure 3.2. Disease and Sex Differences in Gap Characteristics. ......................................... 44

Figure 3.3. Side and Sex Differences in Gap Characteristics .............................................. 45

Figure 4.1. Electromyography Signal Processing................................................................. 55

Figure 4.2. Gap Characteristics Across Frailty Phenotypes. ................................................ 59

Figure 4.3. Burst Characteristics Across Frailty Phenotypes. ............................................. 60

Figure 5.1. Relationship Between Frailty Phenotype and Carbidopa-Levodopa Dose ............... 72

Figure 5.2. Relationship Between Frailty Severity and Daily Physical Activity ....................... 74

Figure 6.1. Relationship Between Frailty, Quality of Life and Parkinson’s Disease................. 84

Figure 7.1. Relationship Between Frailty and Age- and Disease-Related Characteristics.......... 94

Figure 8.1. Physical Function and Quality of Life Outcomes in Parkinson’s Disease.............. 106 


\section{List of Abbreviations}

ABC, activities of balance confidence scale

ADL, activities of daily living

aman, amantadine

ANCOVA, analysis of covariance

ANOVA, analysis of variance

$\beta$, beta coefficient

BB, biceps brachii

$\mathrm{BF}$, biceps femoris

BL, bilateral

BMI, body mass index

CES-D, Center for Epidemiological Studies depression scale

CHSfi, Cardiovascular Health Study frailty index

CI, confidence interval

CL, carbidopa-levodopa

$\mathrm{cm}$, centimeters

COMT, catechol-O methyltransferase

DA, dopamine agonists

EMG, electromyography

enta, entacapone

$\mathrm{F}$, female

FAR, functional axial rotation

FRT, functional reach test

$\mathrm{ft}$, feet

g, grams 
GPS, global positioning system

h, hours

HRT, hormone replacement therapy

kcal, kilocalories

kg, kilograms

km, kilometers

M, male

$\mathrm{m}$, meters

MAO, monoamine oxidase

MF, maximal isometric force

mg, milligrams

min, minute

MLTA, Minnesota Leisure Time Physical Activity Questionnaire

MV, moderate-vigorous

MVE, maximal voluntary exertion

NA, not applicable

NS, non-significant

OR, odds ratio

PD, Parkinson's disease

PDQ-39, Parkinson’s disease Questionnaire - 39

pram, pramipexole

QoL, quality of life

r, correlation coefficient

$\mathrm{R}^{2}$, proportion of variance explained by the model

$\mathrm{R}^{2}$ change, proportion of variance explained by each of the individual criterion 
RFD, rate of isometric force development

rop, ropinirole

S\&E, Swabb and England activities of daily living scale

sec, seconds

TB, triceps brachii

TUG, timed up and go

UPDRS, United Parkinson’s disease Rating Scale

VGF, vertical ground reaction force

$\mathrm{VL}$, vastus lateralis

$\mathrm{VO}_{2}$ max, maximal oxygen capacity 


\section{Acknowledgements}

Sincere gratitude to my supervisors Dr.'s Gareth R. Jones and Jennifer M. Jakobi, who not only supported my academic career, but also included me as part of their family. To Gareth, who motivates me with his enthusiasm to empower older adults to remain active, and to Jenn, who inspires with me her passion for research and her students.

I would like to thank the other members of my committee for their guidance and support; Drs Joan Bottorff and Gord Binsted. Especially, Dr. Colin Powell, whose expertise in Parkinson's disease and frailty has been invaluable to my doctoral experience. I would like to acknowledge the faculty and staff at the School of Health and Exercise Sciences and School of Nursing, who provided mentorship and urged me to approach problems in innovative ways.

Also, thank you to the University of British Columbia, Interior Health Authority, the British Columbia Network for Aging Research, the Government of British Columbia, the Canadian Institutes for Health Research, and Parkinson Society Canada for their financial support of my research and graduate student experiences.

A huge thank you to my friends and fellow students at UBCO; I appreciated your words of advice and the numerous experiences we shared over coffee (or pints!). I am grateful to the yoga community who helped me stay grounded and balanced throughout this experience. To my family, especially Dad, Mom, Dave, and Flo; your love and support has meant so much throughout my academic years. Finally, I give my deepest appreciation to Darc; for your boundless love, encouragement and the sacrifices you made for me. 


\section{Dedication}

To my grandparents, who have helped me see the wisdom and strength that comes with age.

Especially to my Grampy Roland, and the Parkinson’s community, who inspire me to live my fullest life. 


\section{Chapter 1: Introduction and Background}

\subsection{Parkinson’s Disease}

\subsubsection{Disease Onset and Symptom Presentation}

James Parkinson first identified six individual cases of Parkinson’s disease (PD) in his 1817 monograph entitled An Essay on the Shaking Palsy. Today, PD is considered an incurable progressive neurological disorder that affects approximately1\% of Canadians over the age of 65 years. The prevalence of PD will increase in concert with the rapidly growing older adult population, which is expected to rise from 14\% to 23\% by 2030 (Statistics Canada, 2010). The average age of PD onset is 60 years with a mean duration (i.e., diagnosis to death) of 15 years. Incidence rates rise with advancing age, from 17.4 in 100,000 (50 - 59 years) to 93.1 in 100,000 (70 - 79 years) (Lees, Hardy, \& Revescz, 2009). Similar to other neurodegenerative disorders, aging is the primary risk factor for the development of PD (Collier, Kanaan, \& Kordower, 2011). Other contributors to PD pathogenesis include genetic and environmental factors (i.e., rural residency, pesticide exposure) that are both associated with Lewy body formation, oxidative stress and mitochondrial dysfunction (Olanow \& McNaught, 2011; Schapira, 2006).

Idiopathic PD is typically diagnosed by an experienced neurologist through the presence of two of the following cardinal features, in the absence of any other differential diagnoses (Twelves, Perkins, \& Counsell, 2003):

1. Resting tremor, specifically pill-rolling

2. Rigidity, consisting of increased muscle tone in the flexor and extensor muscle simultaneously and “jerky” motions

3. Bradykinesia, referring to slowness of movement (i.e., mask-like face)

4. Postural instability is typically later in the disease progression and described as difficulty with gait and balance, and postural maintenance of body (i.e., holding head). 
PD symptoms typically present unilaterally (“more affected”), usually in the hands or upper limbs, and eventually progresses bilaterally. However, even after the disease progresses to both sides of the body, symptoms are usually more pronounced on the initial side (Djaldetti, Ziv, \& Melamed, 2006; Hallett \& Koshbin, 1980; Kakinuma, Nogaki, Pramanik, \& Morimatsu, 1998; Koller \& Kase, 1986; Nogaki, Fukusako, Sasabe, Negoro, \& Morimatsu, 1995). The selfreported more affected PD side is often weaker than the less affected side, irrespective of hand dominance (Nogaki, Kakinuma, \& Morimatsu, 1999). These PD-associated impairments impact how a person functions during routine activities necessary for independent living. For example, bradykinesia negatively impacts mobility (i.e., walking, transfers), buttoning a shirt or writing a shopping list (Rahman, Griffin, Quinn, \& Jahanshahi, 2008). Persons with PD also face secondary symptoms that impact basic daily function, such as depression, cognitive impairments and non-motor symptoms (Rahman et al., 2008).

The Hoehn \& Yahr scale for PD assesses functional deficits to provide a rating of clinical function (Figure 1.1., Hoehn \& Yahr, 1967). Persons with mild to moderate disease severity (stages 1-3) were included in this research, since the goal was to assess persons living independently in their own home. The Hoehn \& Yahr scale is useful for defining inclusion criteria in a research setting and is highly correlated to motor impairment and disability (Movement Disorder Society Task Force, 2004). Specific to this research, the Hoehn \& Yahr scale demonstrates a strong relationship with quality of life (QoL) because it reflects the impact of PD severity upon daily life (Schrag, 2000). Other measures of PD severity also exist such as the United Parkinson's Disease Rating Scale (UPDRS), which requires clinical training for its application and thus may not be as useful outside a clinic. 


\begin{tabular}{|cl|}
\hline Stage & Description \\
\hline 1 & No sign of disease \\
1.5 & Unilateral symptoms only \\
2 & Bilateral symptoms, no balance impairment \\
2.5 & Mild bilateral symptoms \\
3 & Balance impairment, physically independent \\
4 & Severe disability, but able to walk or stand unassisted \\
5 & Complete dependence for activities of daily living \\
\hline
\end{tabular}

Figure 1.1. The Hoehn \& Yahr Scale

The Hoehn \& Yahr scale indicates disease severity in subjects with PD (Hoehn \& Yahr, 1967).

\subsubsection{Pathophysiology}

The signs and symptoms of PD primarily result from the reduced availability of dopamine, a neurotransmitter produced and released from the substantia nigra. Dopamine is a neurotransmitter that allows for communication between neurons and receptors across neural synapses throughout the brain. Dopamine contributes to the coordination of movement, balance, posture, and muscle tone through the basal ganglia. PD-associated loss of dopamine leads to excessive inhibition paired with increased reduction in excitatory drive of thalamus projections to motor-related areas of the cortex. These abnormal firing patterns within the basal ganglia result in reduced muscle activation, and lead to bradykinesia and muscular weakness (Galvan \& Wichman, 2008; Obeso et al., 2000). Patterns of muscular activation are often measured with electromyography (EMG) (David et al., 2012). Results from laboratory-based investigations propose that muscle activation patterns can be used to distinguish persons with PD from age- and sex-matched healthy controls (Pfann, Buchman, Comella, \& Corcos, 2002; Robichaud, Pfann, Comella, \& Corcos, 2002). Understanding muscle activation in persons with PD is important as 
these changes signal disruptions in physiological capacity, mobility and signal impending frailty. Frailty may be consequential to changes in muscle activation; therefore determining differences in EMG measured muscle activity may provide a method to assess those becoming more functionally dependent, transitioning from low risk (nonfrail) to high risk (frail).

\subsubsection{Disease Management}

As there is yet no cure for PD, the treatment focuses on disease management with the primary goal of maximizing the individual’s QoL (Fitzpatrick, Peto, Jenkinson, Greenhall, \& Hyman, 1997). Symptom relief is often obtained using dopaminergic medication, which is considered the standard pharmacotherapy for PD (Jancovic, 2008). Dopaminergic therapy acts to augment striatal dopamine transition and includes both levodopa and carbidopa. Levodopa is absorbed in the gut and converted into dopamine either in the bloodstream or in the brain. Carbidopa is added to prevent the conversion of levodopa in the periphery before it crosses blood-brain barrier. Dopaminergic therapy may be supplemented by a combination of other medications in an effort to optimize its effectiveness (Lang \& Lees, 2002). Despite its benefits, long-term carbidopa-levodopa use is associated with eventual wearing-off (i.e., where medication becomes less effective and symptoms return) and further motor complications such as dyskinesia (i.e., uncontrollable writhing movements) (Reichmann \& Emre, 2012). Surgical options are available, namely deep brain stimulation that employs high frequency stimulation through an implanted electrode (Olanow, Brin, \& Obeso, 2000); however, such invasive interventions are usually reserved for when the disease and medication side effects become severely disabling.

In persons with $\mathrm{PD}$, exercise is used in conjunction with pharmaceutical therapy to manage the decline in physical function. Chronic exercise improves muscle size, strength and endurance (David et al., 2012; Goodwin, Richards, Taylor, Taylor, \& Campbell, 2008), neuromuscular function (David et al., 2012), mobility (Ebersback et al., 2010), gait (Goodwin et 
al., 2008), balance, postural stability (Dibble, Addison, \& Papa, 2009), self-reported QoL (Dibble, Hale, Markus, Gerber, \& LaStayo, 2009), and spatial memory (Erickson et al., 2011; Nocera, Altman, Sapienza, Okum \& Hass, 2010). Evidence suggests exercise enhances central processing within the neuraxis, improves brain activity in regions that influence cortical output, and helps to modulate muscle activation patterns required for movement and force (David et al., 2012). This emphasizes the importance of using exercise in maintaining physical function throughout the progression of PD. However, there is a paucity of evidence related to the threshold of physical function required for independent daily living and the management of PDsymptoms (Morris \& Schoo, 2004), especially in the early stages of the disease where the greatest success for improving physical function is most evident (Fox, Ramig, Ciucci, Sapir, McFarland \& Farley, 2006; Sutoo \& Akiyama, 2003).

\subsection{Physical Function}

Older adults are living longer with progressive chronic diseases such as PD; thus, there is need to understand the physical function necessary for independent living. Physical function is defined as the ability to perform purposeful movement and tasks necessary for independent daily living, such as dressing oneself (Avlund, Kreiner, \& Schultz-Larsen, 1996). Inter-relationships exist between physiological capacity (i.e., cardiorespiratory fitness, muscular activation), physical activity (i.e., muscular movement, mobility, ambulation), and physical function (i.e., task-based activities required for independent living). Impaired physiological capacity will impact an individual's ability to ambulate independently and/or physical function necessary to complete daily activities. Also, physical inactivity results in declines in physiological capacity, which in turn contributes to lower levels of physical function (Garber \& Friedman, 2003). Examination of physiological capacity during typical daily life is important because it provides critical indicators of functional change between populations, individuals, and/or muscle groups 
(Westerterp, 2009). Thus, examination of physiological capacity and physical activity during real-life activities (i.e. outside of the laboratory or clinical setting) may give a more realistic indication of physical function necessary for independent living.

\subsubsection{Physical Activity}

Physical inactivity is often associated with declining physiological capacity in persons with PD, such as decreased muscular strength and increased muscle fatigability (Garber \& Fredman, 2003; Lou, Kearns, Oken, Sexton, \& Nutt, 2001). Declining dynamic neural control, balance, postural transitions and symptom progression in PD lead to reductions in coordinated mobility (King, 2009) and may result in decreased physical activity participation (Fertl, Doppelbauer, \& Auff, 1993). Physical activity participation during a typical day may indicate physical function necessary for independent living. Available evidence suggests a physically active lifestyle prevents impairments (i.e., disability) and functional limitations (Fiatarone-Singh, 2002). However, to-date the association between physical activity monitored during daily life using an objective tool (accelerometer) and declining physical function, for example in the situation of frailty, has not yet been elucidated in persons with PD. Understanding this relationship may provide evidence toward the development of strategies that stimulate a physically active lifestyle that promotes PD symptom relief (Speelman, van de Warrenburg, van Nimwegen, Petzinger, Munneke, \& Bloem, 2011).

\subsubsection{Muscle Activity}

Assessing physiological capacity, specifically muscle activity, can provide important information about the physical function necessary for independent living in persons with PD. Functional dependence associated with PD result from the inability to direct motor output from the basal ganglia (Hallett \& Koshbin, 1980; Salenius, Bikainen, Kaakola, Hari, \& Brown, 2002). Underlying motor impairments in PD lead to poor balance, limited range of motion, decreased muscular endurance, reduced muscle strength, and loss of muscle tone. The bioelectrical activity 
of muscle, detected using EMG, may reveal valuable information regarding age and diseaseassociated changes in muscle activity and quiescence. Deteriorations in muscular activation and maximal voluntary power generation can predict mobility limitations that influence daily physical function in older adults (Clark, Patten, Reid, Carabello, Phillips, \& Fielding, 2011; Kent-Braun, 2009). Specifically in PD, increased variability in corticospinal activation results in irregular motor unit recruitment and discharge patterns (Glendinning \& Enoka, 1994).

Alterations in muscle activity characteristics are seen in laboratory-based tasks; however, the impact of PD on muscle activity and quiescence required during daily life activities has yet to be determined.

\subsubsection{Implications}

There is growing evidence that prehabilitative interventions are beneficial in preserving or improving physical function of persons who are in the early stages of PD (Hoehn Yahr stages 1-3). Such interventions may help delay functional decline by increasing physiological capacity in the early stages of PD, resulting in increased muscular strength and endurance (Crizzle \& Newhouse, 2006; King \& Horak, 2009). However, the current PD literature does not identify how changes in physical function manifest outside the laboratory or clinical setting. Understanding how changes in physiological capacity influence physical function, while persons with PD engage in their typical daily activities has yet to be examined. The intent of this research is to assess physiological capacity (muscle activity) and physical activity (accelerometer) during independent daily living to examine how they impact physical function (daily living tasks) in persons with PD. The results from these studies may be used to strengthen interventions aimed at improving physiological capacity which in-turn will preserve physical function and QoL in persons with PD (Quinn, Busse, Khalil, Richardson, Rosser \& Morris, 2009). This evidence is most important for females with PD who often experience poorer QoL outcomes than males (Shulman, 2007). 


\subsection{Sex Differences in Parkinson's Disease}

Underlying physiological sex differences may lead to clinical differences in PD symptom expression between males and females. The term 'sex' (male, female) is a multidimensional biological construct that encompasses anatomy, physiology, genes, and hormones (Johnson, Greaves, \& Repta, 2007). Sex is important since biological differences play a role in health and disease processes. Evidence suggests that males are 1.5 times more likely to develop PD than females (Twelves et al., 2003). Sex differences in disease incidence and progression may be attributed to the effect of estrogen on dopaminergic pathways (Miller \& Cronin-Golomb, 2010). Females have naturally higher endogenous-striatal dopamine concentrations than males; therefore the time to reach a threshold of critical depletion where PD symptoms appear may be delayed (Ben Shlomo, 1997; Pohjalainen, Rinne, Nagren Syvalahti, \& Hietala, 1998). Recent evidence suggests estrogen may protect dopaminergic neurons by down-regulating the enzyme Catechol-O-methyltransferase, which metabolizes levodopa and dopamine (Chen et al., 2004; Rubin, 2007; Tsang, Jiang, Ramsden, \& Ho, 2001). These physiological sex differences may serve to explain clinical differences observed between males and females.

Females with PD are an under-represented group in research, yet they experience greater functional decline (Leveille, Penninx, Melzer, Izmirlian, \& Guralnik, 2004; Paganelli et al., 2006; Smith \& Baltes, 1999) and are often rendered frail more so than age-matched males (Fried et al., 2001). Defining frailty has been proposed as an early indicator of impending functional decline and may have implications for quality of life and morbidity in persons with PD (Ahmed, Sherman, \& VanWyck, 2008). This evidence suggests that there is a need to investigate underlying sex differences and the implications of physiological capacity on frailty and daily function in males and females with PD. 


\subsection{Frailty}

Frailty, defined by the Cardiovascular Health Study frailty index (CHSfi), is a meaningful predictor of functional abilities due to its features of weakness, decreased endurance and gait slowness (Fried et al., 2001). Frailty is a multidimensional geriatric syndrome that results from an accumulation and integration of deficits (Powell, 1997), leading to physiological vulnerability and eventual functional dependence (Whiston, Purser, \& Cohen, 2007). Frailty itself cannot be “seen” (Pel-Little, Schuurmans, Emmelot-Vonk, \& Verhaar, 2009) but is commonly characterized by the presence of physical deficits including; weight loss, general weakness and fatigue, sarcopenia, decreased muscular strength and endurance, decreased cardiovascular endurance, decreased bone mass, increased fall-risk, and increasing chronological age (Bales \& Ritchie, 2002; Bandeen-Roche et al., 2006; Brown, Sinacore, Binder, \& Kohrt, 2000; Maggio et al., 2005; Matteini et al., 2008; Paganelli et al., 2006; Pel-Little et al., 2009; Puts, Lips, \& Deeg, 2005; Shardell et al., 2009; Shore \& deLateur, 2007; Syddall et al., 2003; Voznesensky, Walsh, Dauser, Brindisi, \& Kenny, 2009; Woo, Goggins, Sham, \& Ho, 2005). Muscle activation patterns during daily life differ across stages of frailty. Previous investigations have demonstrated decreased muscle activity, specifically burst number and duration, in frail compared to nonfrail older women; this decreased muscle activity was associated with reduced physical activity participation (Theou, Jones, Vandervoort, \& Jakobi, 2010). Changes in muscle activation patterns may represent physiological (i.e., muscle fiber proportion, motor unit firing rate, muscle fatigue) and functional (i.e., force generation, rate of movement) patterns relative to frailty progression (Theou et al., 2010). Other social and cognitive deficits are also recognized as factors associated with frailty (Avila-Funes et al., 2009; Maggio et al., 2005; Roland, Theou, Jakobi, Swan, \& Jones, 2011a). How frailty is defined has an implication for identifying functional dependence in older adult populations. 
Multiple definitions of frailty exist due to the variety of physical, mental and social deficits that relate to its expression (Bergman, Beland, Karunanathan, Hummel, Hogan, \& Wolfson, 2004; Borges \& Menezes, 2011; Levers, Estabrooks, \& Ross Kerr, 2006). In this research, frailty is defined according to the CHSfi as "a biologic syndrome of decreased reserve and resistance to stressors, resulting from cumulative declines across multiple physiologic systems, and causing vulnerability to adverse outcomes" (Fried et al., 2001). The physiologic syndrome of frailty is an independent risk factor for falls, hospitalization and disability. Although a variety of assessment tools exist and differ in how they conceptualize a person as frail, there is no consensus on which criteria best identifies frailty (Cigolle et al., 2009). The CHSfi was employed in this research for its ability to objectively capture physical characteristics associated with early frailty or often referred to as prefrailty. Prefrailty is prevalent in the community (Gill, Gahbauer, Allore, \& Han, 2006) and a significant contributor to functional decline (Fried et al., 2001). Previous investigation determined persons categorized as prefrail are two-and-a-half times more likely to become frail relative to those categorized as nonfrail (OR = 2.63; Fried et al., 2001). Frailty is the result of a reduced physiological capacity, which affects physical function (Fried et al., 2001).

Over $50 \%$ of independent living older adults ( $65^{+}$years) are estimated to express frailty characteristics resulting in functional decline (Gill et al., 2006). Frailty is dynamic, and bidirectional transition between stages of frailty can occur (Figure 1.2.). For example, preventative and restorative interventions can help an adult transition towards lesser frailty, i.e. more independence (Gill et al., 2006). 


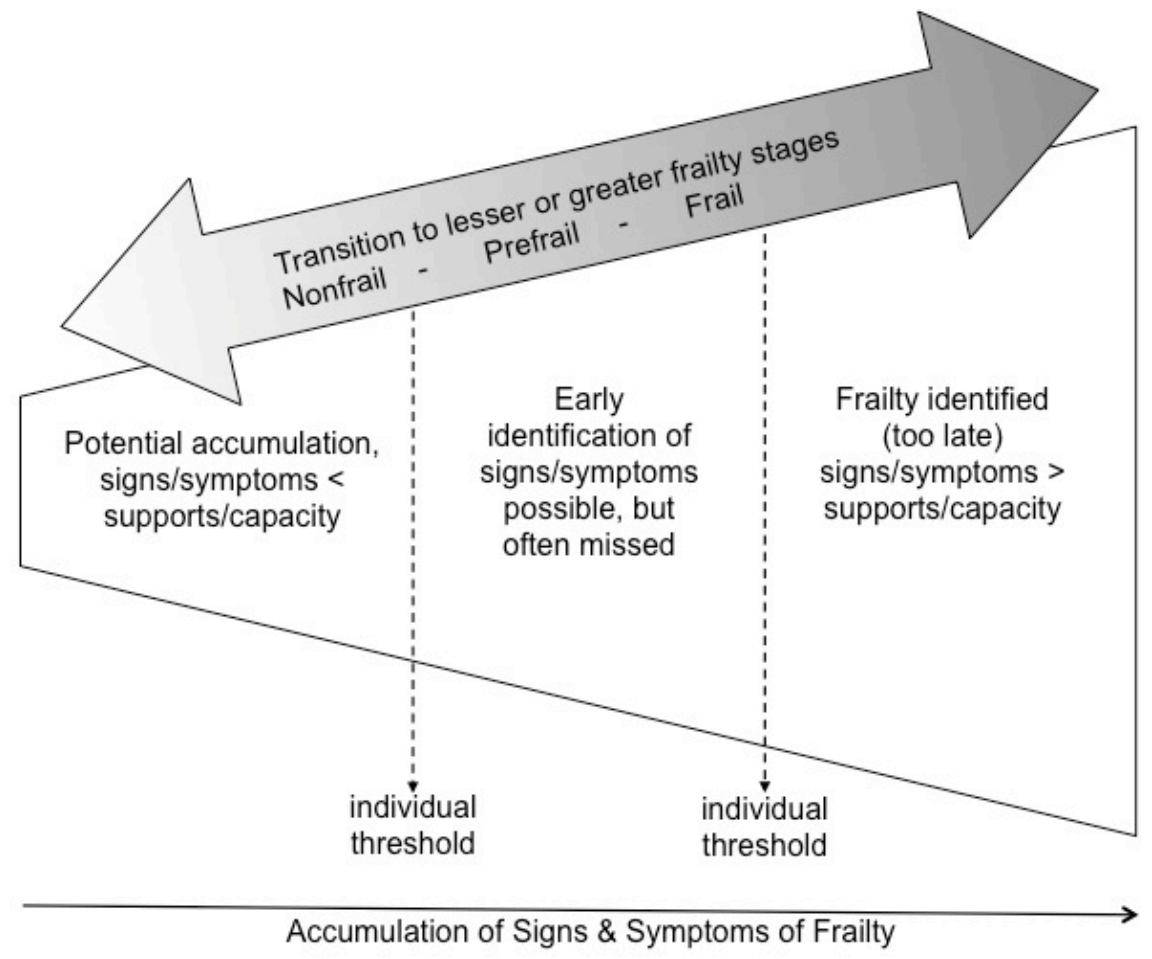

Figure 1.2. Accumulation of Signs and Symptoms Associated with Stages of Frailty

Frailty identification is often missed in its early stages (prefrailty) and is made only once the person has accumulated enough deficits to full transition into frailty.

There is growing concern that healthcare professionals across the community-care continuum likely underestimate or diagnose frailty symptoms too late, given its complex and transitional nature (Bergman et al., 2004; Gobbens, Luijkx, Wijnen-Sponselee, \& Schols, 2010; Roland et al., 2011a). An understanding of factors associated with the onset of frailty is vital, particularly for the early identification of this syndrome (Xue, Fried, Glass, Laffan, \& Chaves, 2008).

\subsubsection{Frailty in Parkinson's Disease}

PD can present as general functional decline, which is common among frail older adults. Assessment of physical function can be used to indicate physical frailty in persons with PD. Frailty and PD share the appearance of physical vulnerability and can coexist in the older adult (Ahmed et al., 2008; Powell, 2008). Persons with PD are typically excluded from scientific research aimed at identifying frailty; thus, there is a paucity of evidence that characterizes frailty within a PD population (Wong, Weiss, Sourial, Karunanthan, Quail, Wolfson \& Bergman, 2010). 
Current definitions of frailty come from the clinical geriatric model (Powell, 1997), which may be very different from the experience of persons with PD who independently reside in their own home (Herrmann, Osiek, Cos, Michel, \& Robine, 2005). This necessitates investigation into the presence of frailty in persons with PD and its impact upon functional tasks required during normal independent daily living. There is limited research available on frailty within PD. Studies suggest persons with PD have a five-fold increased risk for physical frailty compared to the general older adult population, and disease severity is directly related to increased frailty (Ahmed et al., 2008). Since frailty is dynamic (Figure 1.2.) and transitioning to lesser frailty (i.e., greater independence) is possible, frailty-related functional decline may be identified and minimized among persons with PD. However, additional evidence is required to detect and manage frailty in the context of PD.

\subsection{Research Design and Objectives of the Dissertation}

The objective of this dissertation was to examine how changes in physiological capacity and physical activity might influence the level of physical function required for independent living in males and females with PD (Figure 1.3.). Because sex differences in symptom expression and physical function exist in PD, males and females will be considered individually in this dissertation. Frailty is the result of losses in physical function, which is needed to sustain QoL in persons with PD (Muslimovic, Post, Speelman, Schmand, de Haan, \& CARPA study group, 2008). Identification of frailty in the context of PD, especially in females, may contribute to the management of frailty-related declines in physical function and improved QoL. Physical function is influenced by both physiological capacity (i.e., muscle activity) (Clark et al., 2011; Kent-Braun, 2009) and physical activity (i.e., ambulatory movement) (Glendinnings, 1997; LaCroix, Guralnik, Berkman, Wallace, \& Satterfield, 1993; Mor et al., 1989; Seeman, Berkman, Charpentier, Blazer, Albert, \& Tinnetti, 1995) (Figure 1.3.). Therefore in this research, muscle 
activation patterns, measured with EMG, and physical activity, measured with accelerometer and global positioning systems (GPS), were assessed while subjects execute normal daily routines as a means to better determine physical function. Addressing declines in physical function before the loss becomes meaningful (i.e., frailty) will reduce the risk of injurious falls and consequential hospitalization, and ultimately improve the standard of care for persons living with PD (Ashburn, Stack, Pickering, \& Ward, 2001; Mak \& Pang, 2009).

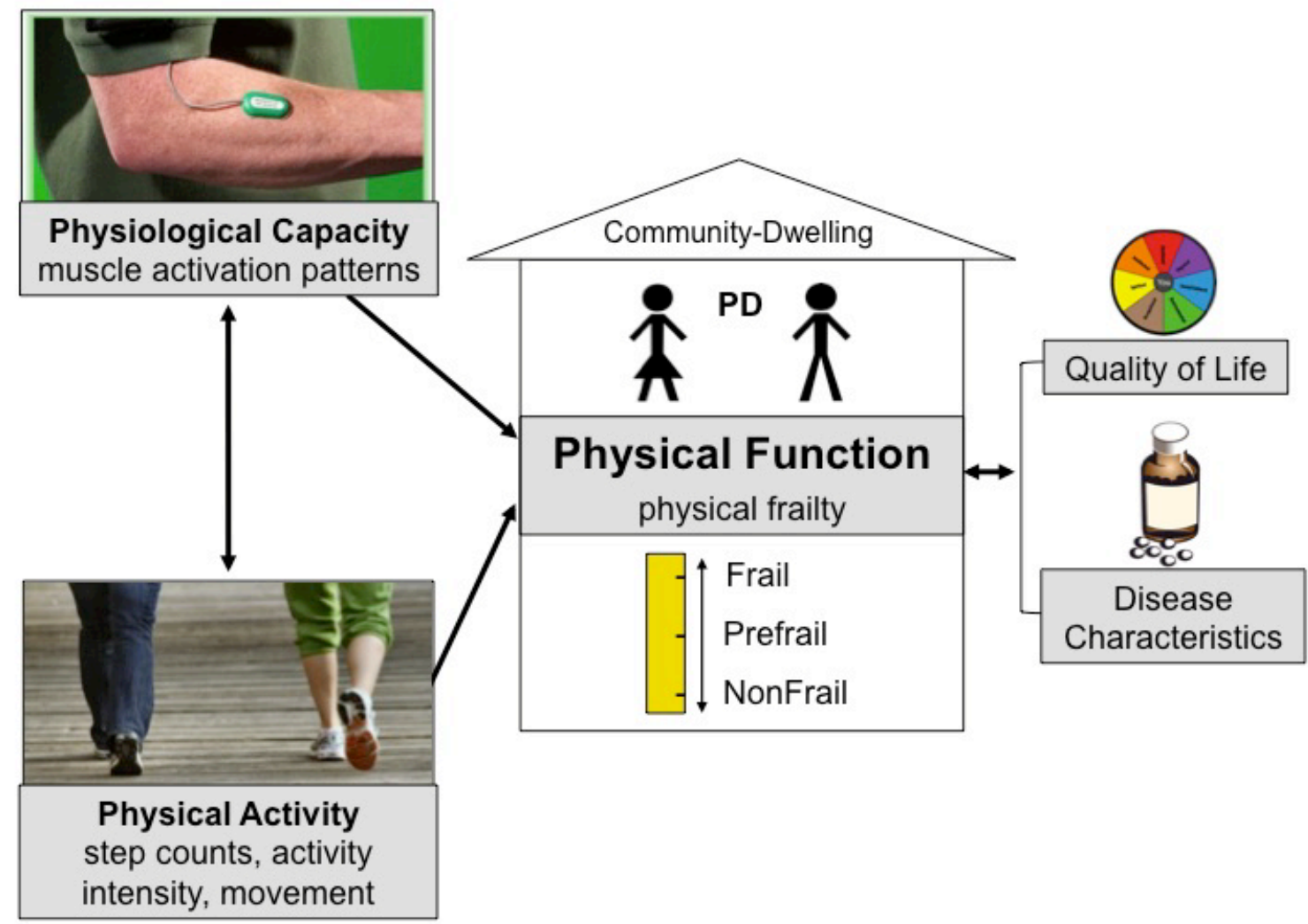

Figure 1.3. Theoretical Framework

This dissertation will examine disease and sex differences in physiological capacity and physical activity in community-dwelling persons with Parkinson's disease (PD) during routine daily activities. The association of physiological capacity and physical activity on frailty will be determined, with consideration for quality of life outcomes. 


\subsubsection{Setting and Recruitment: Measurement of Independent Daily Activities}

Persons with PD living independently in their own home (stages I-III), and age- and sexmatched controls were included as $80 \%$ of the PD population still resides in their own home (Murtch, Strudwick, Roy, \& Downie, 1986). In general, older adults residing in the community live close to functional thresholds where any acute event may render them frail, such as a fallrelated fracture (Gill et al., 2006). However, no study has examined frailty in the context of persons living independently with PD. Laboratory-based studies are limited in that data is collected in a controlled environment and may not account for the multi-joint movement patterns required for independent daily living and environmental factors that fluctuate throughout the day, such as stress and fatigue. This research measures the influence of changes in physiological capacity upon frailty during daily life activities and offers a comprehensive, objective evaluation of physical and muscular activity patterns acquired throughout a normal day.

All data were acquired from a single testing day and based upon the primary research question the outcome measures were segregated across chapters (Figure 1.4.). Subjects were recruited from the Okanagan Valley, greater Vancouver area, and Southwestern Ontario between March 2010 and March 2012. Recruitment presentations and/or email advertisements were distributed to consenting Parkinson’s support groups. Advertisements were placed in local senior shopping centers. Interested subjects contacted the researcher (KPR) and scheduled a testing date and time. Subjects were older than 50 years of age, living independently in their own home, and cognitively intact. Persons with PD were categorized as 1-3 on the Hoehn Yahr disease severity scale and in a steady clinical state (i.e., controlled with medication). All interested subjects met the inclusion criteria, except one female with PD who had mild cognitive impairment and was not included. Daily measures of muscle activation (EMG) and physical activity (accelerometer, GPS) were not collected in three non-PD females (Chapter 7) and daily muscle activation patterns (EMG) were not collected in six non-PD females (Chapter 5). These measures were not 
collected as devices were under repair. Also, recordings of daily muscle activation patterns

(EMG) were unsuccessful for 17 subjects due to equipment error (PD M, n = 7; PD F, n = 4; non $\mathrm{M}, \mathrm{n}=2$; non $\mathrm{F}, \mathrm{n}=4)$.

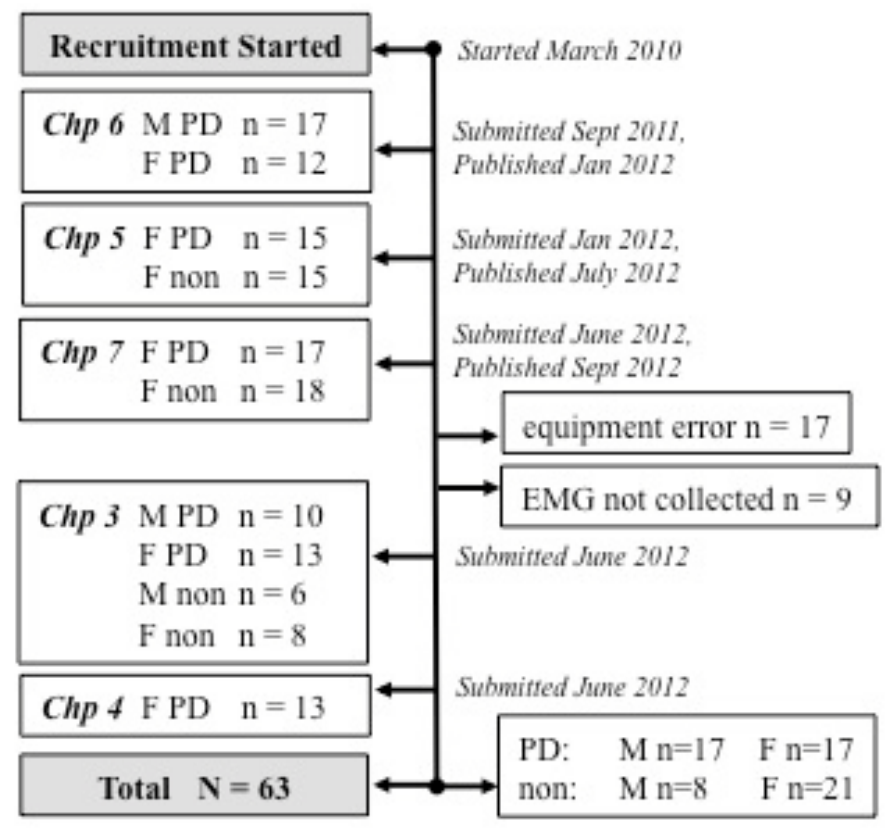

\section{Figure 1.4. Recruitment}

Subjects were recruited from support groups and the local community in the Okanagan Valley, greater Vancouver area and Southwestern Ontario. Individual study samples were taken from this larger recruitment model.

\subsubsection{Variables}

Variables used to describe the sample were sex, age, PD severity (Hoehn \& Yahr scale), self-reported PD duration, medication regimes (i.e., dopaminergic therapy, polypharmacy) and comorbidity. Variables such as being female, older and having increased frailty characteristics all contribute to reduced physical function. The CHSfi was used to classify frailty severity because it assesses physical function characteristics (i.e. grip strength, gait speed) (Fried et al., 2001). Variables that provide an indication of physical function in this study include; physiological capacity, measured as muscle activation patterns (EMG), physical activity (GPS, accelerometer, self-report), and QoL (Parkinson’s Disease Questionnaire-39). Muscle activation was measured using a portable EMG device during a typical 6-7 hour day to assess whether PD 
results in changes to muscle activity patterns during daily life. Muscle activity (EMG) is related to greater physical activity participation and drives physical function at a neuromuscular level, resulting in improved QoL. Similarly, physical inactivity contributes to reduced physiological capacity, which in turn results in functional decline and decreased QoL. Daily physical activity was monitored using a waist-born accelerometer, wrist-born GPS, and self-report [Minnesota Leisure Time activity Questionnaire (MLTQ)]. Physical function is identified as one aspect of PD that impacts QoL (Damiano, Snyder, Strausser, \& William, 1999; Hobson, Holden \& Meara, 1999). The PD Questionnaire-39 (PDQ-39), a self-reported disease-specific assessment tool assessed QoL.

\subsubsection{Objectives and Structure of the Dissertation}

The overall objective of this dissertation was to determine daily physical function and PDspecific factors related to frailty in males and females with PD. To address this: (a) Physiological capacity and physical function during daily life was assessed in persons with PD; (b) Sex differences in clinical PD presentation were evaluated relative to physiological capacity and physical function; (c) Changes in EMG muscle activity patterns that were associated with differences in physical function were examined; and (d) PD-specific factors related to physical frailty were determined. The overall working assumptions were that:

1. PD contributes to functional decline and impacts daily life through changes in muscle activation patterns and increased risk for physical frailty;

2. Females with PD experience greater declines in physical function than males due to differences in PD symptom-expression and increased risk for physical frailty;

3. Daily EMG patterns inform functional mobility, and these muscle activation patterns differ with respect to disease, sex, and frailty phenotype; and

4. Frailty is an assessment of physical function and measures of frailty severity can be used to categorize vulnerability to adverse outcomes in persons with PD. 
Each of the six central chapters of this dissertation research represents a manuscript that has either been published or submitted for publication and answers specific aspects contributing to the overall research question. It was hypothesized that sex differences in PD play a key role in physical function; therefore Chapter 2 provides a systematic review of the current literature.

Chapter 3 quantified gaps in the EMG signal to investigate the hypothesis that daily muscle quiescence is less in persons with PD compared to age- and sex-matched controls and in females with PD compared to males. Chapter $\mathbf{4}$ examined the hypothesis that daily muscle activity increases and muscle quiescence decreases as frailty severity heightens in females with PD. These two chapters examined changes in muscular activation that govern physical function during daily life. Daily physical activity was monitored with accelerometer and GPS and in Chapter 5 the hypothesis that $P D$ influences frailty through decreased physical activity participation in females with $P D$ was examined. Relationships between declining physical function (i.e., frailty) and PD-specific factors were also examined. Decreased QoL (Chapter 6), increased disease severity and resulting therapeutic medication (Chapter 7) were hypothesized to relate to greater frailty severity in persons with $P D$. The final chapter, Chapter $\mathbf{8}$, discusses this cohesive body of work, its significance, limitations and impact.

This project is unique in the fact that it bridges the gap between laboratory-based research and what persons with PD experience during their daily life by examining physiological capacity, physical activity and factors related to frailty (i.e. age- and disease-related characteristics, QoL), while taking sex differences into consideration. Functional decline is complex and transitional in those who reside independently within their own home; however, understanding early indicators of declines in physical function can enhance early recognition, before a crisis occurs. Identifying early signs of functional decline in the initial stages of PD will help direct individual care needs and interventions (i.e., exercise) that may help to restore independence and mitigate further frailty and functional decline (Hammerman, 1999). 


\section{Chapter 2: Factors Related to Functional Independence in Females with Parkinson's Disease: A Systematic Review ${ }^{1}$}

\subsection{Overview}

Parkinson's disease (PD) is a progressive neurological disease affecting $1 \%$ of North American’s 65 years and older (CIHI, 2007). A unifying PD mechanism has yet to emerge (Obeso et al., 2010). The cardinal pathological feature of PD is dopamine neuron degeneration within the substantia nigra pars compacta and subsequent striatal dopamine loss, associated with primary motor features (Lees et al., 2009). Combined genetic, cellular and environmental factors may contribute to cell death and disease pathogenesis (Obeso et al., 2010). In Europe and North America the incidence of PD is 1.5-2 times lower and symptom onset is two years later in females than males (Baldereschi et al., 2000; Ben Shlomo, 1997; Haaxma et al., 2007; Lees et al., 2009; Twelves et al., 2003). Conversely, in Yamagata Japan PD incidence is reported to be greater in females $(91.0 / 100,000)$ compared to males $(61.3 / 100,000)$ (Kimura et al., 2002). Reasons for this diversity have yet to be determined. Evidence suggests that clinical presentation of PD may differ between males and females (Fleming, Tolson, \& Shartau, 2004).

Symptom expression differs between males and females with PD (Table 2.1). Females with PD seek hospital care 1.3 times more than males, frequently from a debilitating fall (Bloem, Grimbergen, Cramer, Williensen \& Zwinderman, 2001; Grimbergen, Munneke, \& Bloem, 2004). Females experience greater fluctuations in PD motor symptoms, specifically; pain, tremor, rigidity, dyskinesia, and instability (Baba, Putzke, Whaley, Wszolek, \& Utti, 2005). These affect motor performance and impact functional ability (Haaxma et al., 2007). Existing reviews describe greater disability and reduced quality of life in women with PD (Shulman,

\footnotetext{
${ }^{1}$ A version of this chapter is published. Roland, K.P., Jakobi, J.M. Powell, C., \& Jones, G. R. (2011b). Factors related to functional independence in females with Parkinson's disease: a systematic review. Maturitas, 69(4), 304311.
} 
2007). Differences in cognition and resulting clinical characteristics (e.g., sleep, behaviour, depression, visuo-spatial, coordination) are attributed to the underlying effect of estrogen on dopamine metabolism (Miller \& Cronin-Golomb, 2010). Pavon and colleagues (2010) review motor and neuropsychiatric symptoms, disability, behaviour, management and social outcomes in women with PD. These authors recommend further investigation into impact of estrogen on pathology, symptoms, and treatment. However, few studies emphasize the influence of sexrelated differences upon physical function, considering its importance for maintaining an independent lifestyle.

Although much is known about PD characteristics, there is a paucity of evidence to explain how biological differences may affect the day-to-day physical function in females with PD. Growing evidence indicates that exercise and physical therapy interventions are beneficial for maintaining and improving physical function in PD (Hirsch, Toole, Maitland, \& Rider, 2003). However, no definitive exercise prescription guidelines for females with PD currently exist (Schenkman, Hall, Kumar, \& Kohrt, 2008).

Although the existing literature highlights 'sex' and 'gender' differences in PD, the terms are often misused. Sex, being either 'male' or 'female', is defined as a multidimensional construct that encompasses anatomy, physiology, genetics, and hormonal regulation, which play critical roles in health and disease (Johnson et al., 2007). Gender research provides important insight into social-cultural influences related to being a ‘man’ or ‘woman’ (Johnson et al., 2007). The focus of this review was to examine sex-related differences for physical function in persons with PD. Inclusion of gender-related differences was beyond the scope of this paper. This systematic review aims to: 1) Identify the specific impact that PD may have on the physical function of females; and 2) Explore opportunities to improve rehabilitation strategies that target biological (sex) differences. 
Table 2.1. Presentation of Parkinson's Disease in Females as Compared with Males

Epidemiology

- Lower general risk and incidence of PD

Clinical Presentation

- More consistently distressing PD symptoms

- $\quad$ Decreased quality of life and increased disability

- $\quad$ Milder motor symptoms, but higher UPDRS rigidity, dyskinesia, instability, and ADL capacity scores.

- $\quad$ Greater prevalence of levodopa-induced dyskinesia

- Tremor is more often at symptom onset

- Higher risk of fracture due to falls

Treatment

- Levodopa-induced dyskinesia due to higher plasma concentrations (i.e. dosage per kg body weight)

- Improvements in the following due to HRT (estrogen):

o on-time

o UPDRS motor and disability score

o disease progression (i.e. slows)

o cognitive performance

o levodopa-induced dyskinesia

o substantianigra dopamine cells (i.e. protects against loss).

ADL, Activities of Daily Living; HRT, Hormone Replacement Therapy; kg, kilogram; PD, Parkinson’s disease; UPDRS, United Parkinson’s Disease Rating Scale. Reprinted with permission from Maturitas.

\subsection{Methods}

\subsubsection{Literature Search Strategy}

The following electronic databases were searched, following PRISMA 2009 guidelines: PubMed (1980-), Web of Science, EBSCO, SportDISCUS (1980-), PsycINFO (1806-), CINAHL (1982), Ageline (1978-), Embase (1974-), MEDLINE (1950-). Studies were also identified through manual screening of reference lists for relevant papers and personal citation databases. MeSH search terms and key words included: Parkinson's disease, exercise therapy, exercise, functional assessment, physical therapy modalities, women, woman, and female. Studies were selected for abstract-review based on meeting criteria:

1. PD (idiopathic) diagnosis,

2. Functional ability (e.g. balance, range-of-motion, strength, aerobic endurance, ambulation) assessed,

3. Female-specific results reported. 
The principal investigator (KPR) reviewed all full-text articles that met selection criteria to determine inclusion. A secondary check was performed by six independent blinded reviewers who were randomly assigned 25-28 articles included for screening. All blinded reviewers were in complete agreement (100\%) with the selected studies.

\subsubsection{Data Extraction and Quality Analysis}

Key data were extracted from identified papers, including subject characteristics, inclusion criteria, intervention description (i.e., duration, frequency, intensity), outcomes, design, and female-specific results. Quality was assessed using the Checklist for the Assessment of Study Quality (Downs \& Black, 1998), which consists of 26-items in categories: 1) Unbiased information reporting; 2) External validity, generalizability of results; 3) Measurement bias; 4) Confounding recruitment bias; and 5) Sufficient statistical power. Maximum score was 32 points. 


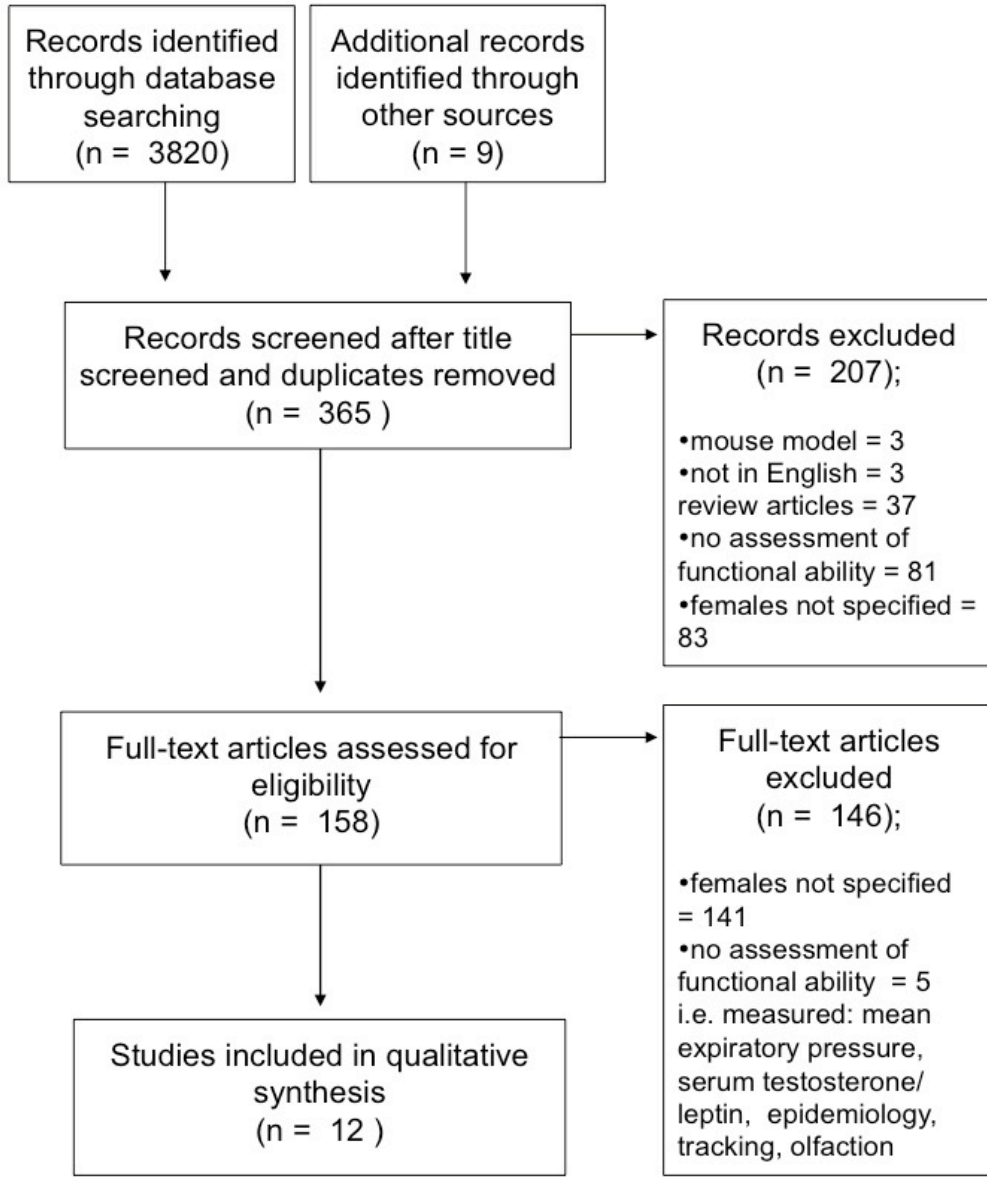

Figure 2.1. Flow Diagram of Study Inclusion According to PRISMA Statement Reprinted with permission from Maturitas.

\subsection{Results}

\subsubsection{Literature Search}

Total of 3845 citations (1980-June 2010) were found. Titles were screened for inclusion criteria and entered into RefWorks (Bethesda, ML) where duplicates were removed (n=365). Abstracts were assessed and full-text reviews were completed on 158 articles that met inclusion criteria. The majority of full-text reviewed studies $(n=146)$ did not specifically report female results. This review identified 12 studies, published from 1997 to 2008 (Figure 2.1.) (Table 2.2.). 
Table 2.2. Results of the Systematic Literature Review

\begin{tabular}{|c|c|c|c|c|c|c|c|c|c|c|c|}
\hline & $\begin{array}{l}\text { First } \\
\text { Author }\end{array}$ & Year & $\begin{array}{l}\mathrm{N}(\mathrm{F}) \\
\text { with } \\
P D\end{array}$ & $\begin{array}{l}\text { Mean } \\
\operatorname{Yrs}(S D)\end{array}$ & $\begin{array}{l}\text { Hoehn } \\
\text { \&Yahr }\end{array}$ & Design & $\begin{array}{l}\text { Length } \\
\text { weeks }\end{array}$ & $\begin{array}{l}\text { Freq } \\
\text { /week }\end{array}$ & $\begin{array}{l}\text { Outcome } \\
\text { Measure }\end{array}$ & $\begin{array}{l}\text { Women-Specific } \\
\text { Conclusions }\end{array}$ & $\begin{array}{l}\text { Quality } \\
\text { Score }^{\mathrm{a}}\end{array}$ \\
\hline \multirow[t]{4}{*}{ Gait } & Kokko & 1997 & $\begin{array}{l}40 \\
(13)\end{array}$ & 67 & $1-3$ & Cohort & NA & NA & $\begin{array}{l}\text { Walk speed, } \\
\text { Cadence, } \\
\text { Stride length }\end{array}$ & $\begin{array}{l}\text { Greater cadence } \\
\text { Shorter stride length }\end{array}$ & 25 \\
\hline & Pedersen & 1997 & $\begin{array}{l}25 \\
(11)\end{array}$ & $\begin{array}{l}M=63.4 \\
F=64\end{array}$ & $1-3$ & Cohort & NA & NA & $\begin{array}{l}\text { Stride frequency, } \\
\text { velocity, torque, } \\
\text { contraction } \\
\text { (output) }\end{array}$ & $\begin{array}{l}\text { Lower stride frequency } \\
\text { Note; men had lower } \\
\text { eccentric contraction } \\
\text { torque output, stronger } \\
\text { isometric and eccentric } \\
\text { contractions, and higher } \\
\text { Webster rating }\end{array}$ & 17 \\
\hline & Morris & 1999 & 1 & 71 & 2.5 & Case & 1 & NA & $\begin{array}{l}\text { 3D trajectories } \\
\text { and ground } \\
\text { reaction force } \\
\text { (kinematic/ } \\
\text { kinetic profiles), } \\
\text { Clinical stride } \\
\text { analyzer (spatio- } \\
\text { temporal } \\
\text { variables) }\end{array}$ & $\begin{array}{l}\text { Reduced speed } \\
\text { Shortened stride length } \\
\text { Reduced ankle power } \\
\text { generation }\end{array}$ & 9 \\
\hline & Lamberti & 1997 & $\begin{array}{l}100 \\
(30)\end{array}$ & $61(9.1)$ & $1-3$ & Cohort & NA & NA & Freezing & $\begin{array}{l}\text { More frequent freezing of } \\
\text { gait }\end{array}$ & 20 \\
\hline \multirow[t]{2}{*}{$\begin{array}{l}\text { Lower } \\
\text { limb force }\end{array}$} & Pääsuke & 2002 & $\begin{array}{l}14 \\
(14)\end{array}$ & $72.6(2.2)$ & $1-3$ & Cohort & NA & NA & $\begin{array}{l}\text { VRT, chair rise } \\
\text { time, RFD, } \\
\text { VGRF, MF }\end{array}$ & $\begin{array}{l}\text { Longer VRT and lower } \\
\text { maximal RFD and MF in } \\
\text { PD. Chair rise time } \\
\text { correlated negatively } \\
\text { with MF, VGRF } \\
\text { positively correlated with } \\
\text { MF and RFD when rising } \\
\text { from a chair. }\end{array}$ & 20 \\
\hline & Pääsuke & 2004 & $\begin{array}{l}12 \\
(12)\end{array}$ & $74.3(6.9)$ & $1-3$ & Cohort & NA & NA & $\begin{array}{l}\text { MF, chair rise } \\
\text { time, VGRF, } \\
\text { RFD, }\end{array}$ & $\begin{array}{l}\text { Lower BL and MF } \\
\text { relative to body mass, } \\
\text { higher BL strength } \\
\text { deficits, and lower max } \\
\text { VGRF in PD. }\end{array}$ & 21 \\
\hline $\begin{array}{l}\text { Spinal } \\
\text { flexibility }\end{array}$ & Schenkman & 2000 & $\begin{array}{l}56 \\
(14)\end{array}$ & $70.7(7.4)$ & $2-3$ & Cohort & NA & NA & $\begin{array}{l}\text { FAR, FRT, 10m } \\
\text { walk, 360-turn, } \\
\text { supine to stand. }\end{array}$ & $\begin{array}{l}\text { Decreased functional } \\
\text { reach }\end{array}$ & 20 \\
\hline
\end{tabular}


Table 2.2. continued...

\begin{tabular}{|c|c|c|c|c|c|c|c|c|c|c|c|}
\hline & $\begin{array}{l}\text { First } \\
\text { Author }\end{array}$ & Year & $\begin{array}{l}\mathrm{N}(\mathrm{F}) \\
\text { with } \\
P D\end{array}$ & $\begin{array}{l}\text { Mean } \\
\operatorname{Yrs}(S D)\end{array}$ & $\begin{array}{l}\text { Hoehn } \\
\text { \&Yahr }\end{array}$ & Design & $\begin{array}{l}\text { Length } \\
\text { weeks }\end{array}$ & $\begin{array}{l}\text { Freq } \\
\text { /week }\end{array}$ & $\begin{array}{l}\text { Outcome } \\
\text { Measure }\end{array}$ & $\begin{array}{l}\text { Women-Specific } \\
\text { Conclusions }\end{array}$ & $\begin{array}{l}\text { Quality } \\
\text { Score }^{\mathrm{a}}\end{array}$ \\
\hline $\begin{array}{l}\text { Spinal } \\
\text { flexibility }\end{array}$ & Schenkman & 2008 & $3(1)$ & 61.3 & $2-2.5$ & Case & NA & NA & $\begin{array}{l}\text { FAR, FRT, 10m } \\
\text { walk, 360-turn, } \\
\text { standing. }\end{array}$ & $\begin{array}{l}\text { Decreased functional } \\
\text { reach }\end{array}$ & 20 \\
\hline $\begin{array}{l}\text { Cardio- } \\
\text { respiratory } \\
\text { Endurance }\end{array}$ & Stanley & 1999 & $20(7)$ & $\begin{array}{l}M=64 \\
W=65\end{array}$ & $2-3$ & Cohort & NA & NA & $\begin{array}{l}\mathrm{VO}_{2} \text { max, time } \\
\text { to maximal } \\
\text { exercise (min) }\end{array}$ & $\begin{array}{l}\text { Higher } \mathrm{VO}_{2} \max \\
* \text { Note } \mathrm{VO}_{2} \text { max values } \\
\text { probably indicate } \mathrm{VO}_{2} \\
\text { peak }\end{array}$ & 19 \\
\hline \multirow[t]{2}{*}{$\begin{array}{l}\text { Estrogen } \\
\& \text { motoric } \\
\text { function }\end{array}$} & Kompoliti & 2000 & $\begin{array}{l}10 \\
(10)\end{array}$ & $42.3(5.8)$ & $1-3$ & Cohort & 5 & NA & $\begin{array}{l}\text { UPDRS (motor, } \\
\text { behaviour, ADL) } \\
\text { S\&E (ADL) }\end{array}$ & $\begin{array}{l}\text { Trends indicate serum } \\
\text { levels of estrogen } \\
\text { correlated with UPDRS } \\
\text { motor subscale }\end{array}$ & 26 \\
\hline & Tsang & 2000 & $\begin{array}{l}20 \\
(20)\end{array}$ & $\begin{array}{l}65.3(4.9) \\
66.9(5.5)\end{array}$ & $1-3$ & $\begin{array}{l}\text { Randomized } \\
\text { controlled } \\
\text { trial }\end{array}$ & 8 & 7 & $\begin{array}{l}\text { Diary, } \\
\text { UPDRS, } \\
\text { Timed tapping, } \\
\text { Hamilton } \\
\text { Depression Scale }\end{array}$ & $\begin{array}{l}\text { Mean 'on' ( } 7 \%) \text { and 'off' } \\
\text { (4\%) time and UPDRS } \\
\text { motor subscale score ( } 3.5 \\
\text { pt) improved in estrogen- } \\
\text { treated group }\end{array}$ & 31 \\
\hline Balance & Venglar & 2005 & $\begin{array}{l}1(1) \\
(+1 \\
\text { M } \\
\text { MSA) }\end{array}$ & 63 & 2 & Case & 8 & $\begin{array}{l}2-1 \\
\text { homebased }\end{array}$ & $\begin{array}{l}\text { Self-report: fall } \\
\text { frequency, loss } \\
\text { of balance, } \\
\text { medication } \\
\text { TUG } \\
\text { ABC } \\
\text { FRT }\end{array}$ & $\begin{array}{l}\text { Drop (51 to } 40 \% \text { ) balance } \\
\text { confidence (ABC) } \\
\text { Decrease in time and } \\
\text { steps (TUG) } \\
\text { Improved FRT } \\
\text { Tai Chi improved her } \\
\text { awareness (how to 'un- } \\
\text { freeze' and aware of } \\
\text { falling) }\end{array}$ & 15 \\
\hline
\end{tabular}

$\mathrm{ABC}=$ Activities of Balance Confidence Scale, $\mathrm{ADL}=$ activities of daily living, $\mathrm{BL}=$ bilateral, $\mathrm{F}=$ female, FAR $=$ functional axial rotation, FRT = functional reach test, $\mathrm{M}=$ male, $\mathrm{MF}=$ maximal isometric force, $\mathrm{NA}=$ not applicable, $\mathrm{PD}=$ Parkinson's disease, $\mathrm{RFD}=$ rate of isometric force development, $\mathrm{S} \& \mathrm{E}=\mathrm{Swabb}$ and England activities of daily living scale, TUG = Timed-Up and Go, UPDRS = United Parkinson's disease rating scale, VGF = vertical ground reaction force, $\mathrm{VO}_{2}=$ volume of oxygen. a. (Downs \& Black, 1998). Reprinted with permission from Maturitas. 


\subsubsection{Subjects}

The 12 studies included 302 subjects; 134 (44\%) were female (mean age $=65.5 \pm 8.3$ ) with PD. Subjects were diagnosed with mild to moderate idiopathic PD, as per Hoehn \&Yahr assessment tool (stages I-III, Hoehn \& Yahr, 1967; Ramaker, Marinus, Stiggelbout, \& van Hilten, 2002). All subjects were in a steady clinical state (i.e., controlled by medication) and testing intervals were consistent within medication regimes (i.e., one to three hours after anti-PD medication).

\subsubsection{Study Design}

Eight observational reports (Kokko, Paltamaa, Ahola, \& Malkia, 1997; Kompoliti, Comella, Jaglin, Leurgans, Raman, \& Goetz, 2000; Lamberti et al., 1997; Pääsuke, Mottus, Ereline, Gapayeva, \& Yaba, 2002; Pääsuke, Ereline, Gapeyeva, Joost, Mottus, \& Taba, 2004; Pedersen, Oberg, Larsson, \& Lindval, 1997; Schenkman, Morey, \& Kuchibhatla, 2000; Stanley, Protas, \& Jankovic, 1999), three case studies (Morris, McGinley, Huxham, Collier, \& Iansek, 1999; Schenkman et al., 2008; Venglar, 2005) and one randomized controlled trial (Tsang, Ho, \& Lo, 2000) met the inclusion criteria. Duration ranged from single assessments $(n=4)$ to a 64week exercise intervention. Outcome measures of functional ability included; gait $(n=4)$, lower limb strength $(n=2)$, spinal flexibility and balance $(n=1)$, cardiorespiratory $(n=1)$, estrogen and motoric function $(\mathrm{n}=1)$. Three studies included an intervention: (1) Cycling for aerobic exercise - case study of two males and one female with PD (Schenkman et al., 2008); (2) Tai Chi for balance - case study of female with PD (Venglar, 2005); and (3) Estrogen supplementation on motoric function-randomized-control trial (Tsang et al., 2000) (Table 2.2.). 


\subsubsection{Quality Analysis}

Scores on the quality checklist ranged from 9/32 (Morris et al., 1999) to 31/32 (Tsang et al., 2000), mean score was $20 \pm 5.4$ (Table 2.2.). Studies that received low $(0-16, \mathrm{n}=2)$ and moderate $(17-25, n=8)$ scores did not report; recruitment, blinding, randomization, intentionto-treat analysis, adverse events and follow-up characteristics (Table 2.2.).

\subsubsection{Outcome Measures}

\subsubsection{Functional Capabilities}

Gait. Kokko et al. (1997) examined postural locomotion, balance, stride length, and cadence in 40 subjects ( 13 females, mean age $=67$ years, range $35-84$ ) with PD (mean duration $\mathrm{n}=5.5$ years, range $2-19)$. Investigators reported ambulatory cadence (143 steps/minute) and shorter relative stride length $(1.7 \mathrm{~m}$ ) was greater in females than males (132 steps/minute, $1.9 \mathrm{~m}$; $\mathrm{p}<0.05)$.

Pedersen et al. (1997) assessed cadence and stride length in 14 males (mean age $=63.4$ years, range $50-69$ ) and 11 females (mean age $=64$ years, range $60-69$ ) with PD (duration 1 - 8 years) age-matched to 37 healthy active seniors (18 females). Cadence was significantly slower in females with PD at maximum velocity than both controls and males with PD.

Morris et al. (1999) examined the stride length, gait speed of a 71-year old female with PD (duration 20 years). Results were compared to an age-and height-matched female. The female with PD had slower gait speed (56 vs. $75 \mathrm{~m} / \mathrm{min}$ ) and shortened stride length ( 0.97 vs. 1.3 m) compared to her peer.

Lamberti and colleagues (1997) assessed the occurrence of freezing of gait and fall characteristics (questionnaire) in 100 people with PD (30 females, mean age $=61 \pm 9.1$ years, mean duration $=6.5 \pm 4.0$ years). In this study, freezing of gait occurred more frequently ( $<<$ 
$0.05)$ in females $(70 \%, \mathrm{OR}=1.50[\mathrm{CI} 1.28-1.61])$ than males $(55 \%$, OR $=0.66$ [CI $0.61-$ 3.63]) with PD. The results did not differ significantly when comparing on and off medication. Lower Limb Strength. Pääsuke et al. (2002) investigated the relationship between force preparation and production in 14 females with $\mathrm{PD}$ (mean age $=72.6 \pm 2.2$ years, mean duration $=$ $10.3 \pm 1.2$ ) compared to 12 'healthy’ age-matched females (mean age $=72.8 \pm 0.8$ ). Females with PD had a 12\% prolonged reaction time in the right leg and 14.8\% in the left. Maximum isometric force was $15.3 \%$ less in the right and $22.1 \%$ in the left compared to controls $(\mathrm{p}<0.05)$ (Pääsuke et al., 2002). This may reflect postural asymmetry in PD, or the contribution of cerebral lateralization to lower leg function (Geurts, Boonstra, Voermans, Dienders, Weendesteyn, \& Bloem, 2011).

In a subsequent study, Pääsuke et al. (2004) measured bilateral isometric leg-extension force and chair-rise performance in 12 females with PD (mean age $=74.3 \pm 6.9$ years, mean duration $=10.7 \pm 4.5$ years) compared to 16 healthy age-matched females (mean age $=71.7 \pm$ 4.4 years). Females with PD exhibited $19.4 \%$ weaker bilateral maximal force, $29.8 \%$ slower rate of force development, and took 24.4\% longer to complete chair-rise assessment than controls (p $<0.05)$.

Pedersen, as described above, assessed ankle dorsi-flexion of the tibialis anterior and triceps surae. They reported males had stronger isometric and eccentric contractions of ankle dorsi-flexors ( $\mathrm{p}<0.03$ ) than females with PD (Pedersen et al., 1997).

Spinal Flexibility and Balance Control. Schenkman (2000) measured spinal flexibility (Functional Axial Rotation), and dynamic balance (Functional Reach), timed supine to standing, 10-meter walk, and 360-degree standing turn in people with $(\mathrm{n}=56,14$ females, mean age $=$ $70.7 \pm 7.4$ years $)$ and without $(n=195,129$ females, mean age $=71.4 \pm 5.0$ years $)$ PD. Spinal flexibility was moderately correlated to functional limitations $(\mathrm{R}=0.488 ; \mathrm{p}=0.0001)$. 
Regression analysis revealed that being female contributed significantly to poor spinal flexibility and balance control in PD.

Cardiorespiratory Fitness. Stanley and colleagues (1999) compared maximal oxygen consumption $\left(\mathrm{VO}_{2} \mathrm{max}\right)$ and exercise tolerance during stationary cycling in males $(\mathrm{n}=13$, mean age $=64 \pm 7$ years, mean duration $=7.4 \pm 4$ years $)$ and females $(n=7$, mean age $=65 \pm 8$ years, mean duration $10.6 \pm 4.4$ years) with PD relative to healthy age-matched males $(n=7$, mean age $=66 \pm 6$ years $)$ and females $(n=16$, mean age $=66 \pm 8$ years $)$. No significant differences for $\mathrm{VO}_{2}$ max were observed between males $\left(23.5 \mathrm{~mL} \cdot \mathrm{kg}^{-1} \cdot \mathrm{min}^{-1}\right)$ and females $\left(20.1 \mathrm{~mL} \cdot \mathrm{kg}^{-1} \cdot \mathrm{min}^{-1}\right)$ with PD. Also, individuals with PD did not differ significantly from healthy males $\left(25.5 \mathrm{~mL} \cdot \mathrm{kg}^{-}\right.$ $\left.{ }^{1} \cdot \mathrm{min}^{-1}, \mathrm{p}=0.50\right)$ and females $\left(16.2 \mathrm{~mL} \cdot \mathrm{kg}^{-1} \cdot \mathrm{min}^{-1}, \mathrm{p}=0.35\right)$. Although not significantly different, males (9.5 minutes) and females (5.2 minutes) with PD were unable to tolerate the exercise test as long as male (13.1 minutes, $\mathrm{p}=0.02)$ and female $(5.4$ minutes, $\mathrm{p}=0.20)$ controls (Stanley et al., 1999). Problems in interpreting study results stem from; low statistical power, varying fitness levels (i.e., female controls had poor cardiorespiratory fitness) and it is unlikely the subjects achieved true $\mathrm{VO}_{2} \max$ (Stanley et al., 1999). The poor cardiorespiratory scores in the controls might suggest another underlying pathology; therefore, they may not represent a healthy comparison.

Estrogen and motoric function. Estrogen may influence PD symptoms in premenopausal females. Kompoliti (2000) investigated ten premenopausal females (mean age $=42.3 \pm 5.8$ years) prospectively during their 'off-state' for five weeks. Menstruating females with PD experienced cyclical changes in their motor skills, as measured by the UPDRS (mean change $=$ $10.9 \pm 6.4)$, although changes were not correlated with serum levels of estrogen $(p=-0.09)$.

Although the evidence presented is scarce, existing research demonstrates that functional ability is likely limited in females with PD. Results present restrictions in gait, lower-limb 
strength and balance in females with PD. This suggests that further investigation into physiological patterns and interventions specific to females with PD is warranted.

\subsubsection{Intervention Studies}

Balance. Venglar (2005) administered a twice-weekly (one group, one home) Tai Chi intervention for eight-weeks. This single-subject case-study of a 63-year old female diagnosed with PD (duration = 20 years) had previously reported frequent loss of balance and falls. Immediately post group-intervention (eight-weeks), the subject reported further loss of balance (ABC -20\%). However, the subject continued to practise Tai Chi at home 3-5 times per week following the conclusion of group-exercise, and improved her Activities Balance Confidence scale score by $40 \%$ (20\% net gain) at two-month follow-up (Venglar, 2005). Also, the subject reduced her Timed-Up and Go time (12.3 to 10.6 seconds) and number of steps (17 to 14 steps) immediately post-intervention. Authors reported improvements in Functional Reach scores, although no values were given. Finally, the subject self-reported that Tai Chi improved her ability to regain movement after freezing of gait and increased fall-risk awareness (Venglar, 2005).

Aerobic endurance. Schenkman and colleagues (2008) studied two males (60, 72 years) and one female (52 years) with PD as part of a larger randomized controlled trial of a four-month aerobic endurance exercise program. The female’s UPDRS performance declined during the study; however, Continuous-Scale Physical Functional Performance balance score (16.7\%), $\mathrm{VO}_{2} \max (8.5 \%)$ and 6-minute-walk distance (16.3\%) all improved, but no statistical analysis was performed (Schenkman et al., 2008). Additionally, functional axial rotation improved on the right $\left(+2.5^{\circ}\right)$, but declined on the left - side $\left(-20^{\circ}\right)$, demonstrating the often-asymmetric symptom expression characteristic of PD. This suggests that exercise may have improved physical fitness but not delay disease progression in this female with PD. 
Estrogen Supplementation and Motoric Function. Tsang et al. (2005) investigated lowdose estrogen in relation to motoric function in 40 postmenopausal females with PD (> two years prior menstruation). Females were randomized to either low-dose estrogen (Premarin, $0.625 \mathrm{mg}$ ) or placebo daily for eight-weeks. Mean self-reported 'on-time’ increased (7\%) and 'off-time' decreased (4\%) and UPDRS motor subscale improved (3.5 points) compared to placebo ( $\mathrm{p}<$ 0.05) (Tsang et al., 2005).

\subsection{Discussion}

How PD impacts females is currently under reported which is of concern, given that females often cite greater disability and reduced quality of life (QoL) compared to males (Shulman, 2007). Since no cure exists, PD management in females should focus on the maintenance of functional independence and quality of life. This review examined 12 studies that reported on the functional abilities of females with PD. Albeit equivocal, the current literature suggests that females with PD have different gait patterns compared to age-matched healthy females and males with PD. In addition, these studies reported poor balance confidence and increased freezing of gait in females as compared to males with PD. Females experience lower exercise tolerance compared to males with PD and controls. Evidence suggests that lowdose estrogen in postmenopausal females with PD may improve duration of medication effectiveness ('on-time') and UPDRS motor scores. Although the studies offer insight into gait, balance, and motor function characteristics in females with PD, these insights are limited due to methodological concerns.

The studies presented used a variety of methodologies and measured different functional outcomes, making it difficult to make comparisons. To make conclusive statements regarding the specific impact of PD on females is premature, and these conclusions should be interpreted with caution. Future investigations, sufficiently powered with equal sex-distributions would enable 
examination of sex differences in functional limitations, and the contribution of female physiology to PD symptom expression. In addition, these results may reflect significant age differences in PD population, as females are typically older (Twelves et al., 2003).

Results support sex differences in PD gait (Pedersen et al., 1997). Cadence was inconsistently reported in females compared to males with PD (Kokko et al., 1997; Morris et al., 1999) and healthy controls (Pedersen et al., 1997; Morris et al., 1999). Subjects achieved speeds differently; females took more steps with shorter stride lengths compared to males (Kokko et al., 1997). Biomechanical sex differences, physical condition and/or stage of PD may account for the variance. Freezing of gait occurs more frequently in females, especially as PD progresses (Lamberti et al., 1997).

Lower body muscle strength and size declines with age, especially in combination with decreasing bone density in females; this is associated with falls, fracture and functional dependence (Hughes et al., 2001; Taggart \& Crawford, 1995). Pääsuke and colleagues (2002, 2004) reported slowed reaction time, reduced force generation, and increased chair-stand time in knee-extensors of females with PD compared to age-matched controls. Chair-stand assessment is clinically important and directly applicable to UPDRS. This suggests that females with PD experience greater loss of lower-body muscle strength compared to controls, increasing fall-risk. Isolated laboratory-based electromyography in knee-extension and elbow-flexion show low frequency activation (5-15Hz) and deficits in modulating muscle activation in persons with PD, especially females (Pfann et al., 2001; Robichaud, Vaillancourt, Comella, \& Corcos, 2005). Electromyography may determine the influence of muscular strategies on gait and balance control, and thus provide further clinical information relative to functional mobility in persons with PD. 
Sex contributed significantly to dynamic balance (Schenkman et al., 2000). Ankle strength contributes to postural control and may explain poor balance control in females with PD. Longer balance interventions may improve confidence, kinesthetic awareness and balance abilities; although current evidence is speculative (Pfann et al., 2001). It is unclear whether benefits result from improved balance or confidence (Wolf, Barnhart, Ellison, Coogler, \& Atlanta FICSIT group, 1997).

Persons with PD reach exercise tolerance thresholds earlier than age-matched controls; biomechanical inefficiency caused by rigidity and slowed movement initiation may contribute (Stanley et al., 1999). Unfortunately, sex differences are unclear due to limited female subjects. Endurance exercise training improved ambulatory economy in males and females with PD; however, females were less efficient than males with PD and controls (Stanley et al., 1999). This is consistent with reports that persons with PD have 20\% higher energy expenditure than ageand sex-matched controls (Protas, Stanley, \& Jankovic, 1996).

Fluctuating estrogen levels in premenopausal females with PD were not directly related to function (Kompoliti et al., 2000). However, in postmenopausal females, daily low-dose estrogen significant improved on-time and UPDRS motor ability (Tsang et al., 2000). Future investigations should examine estrogen therapy on motor ability, dosage, and long-term implications (Jong, Comella, \& Jaglin, 1995).

\subsubsection{Recommendations}

Future studies should emphasize the potential for sex differences and consider improved methodology (i.e., sex-matched recruitment, follow-up). In addition, investigators should carefully consider the use of 'sex' and 'gender' within their research, as they both have distinct meanings. Here we emphasize sex differences, since biology influences PD symptoms and ultimately impacts the female's ability for independent function and substantially reduces her 
QoL as compared to males with PD. Rehabilitation guidelines need to define the specific dimensions of exercise (i.e., frequency, intensity, time, type) most effective on females with PD.

\subsection{Final Remarks}

Clinicians make decisions on appropriate treatment options for individuals with PD. This review presents variables that may influence PD expression in females, yet acknowledges that only a small portion of the literature considers disease implications on females. Also, the heterogeneous nature of PD makes it difficult to generalize these findings to all females with PD, whose symptoms and experiences may differ. Additionally, the importance of cognitive, environmental, metabolic and peripheral factors requires further consideration (Hughes et al., 2001; Miller \& Cronin-Golomb, 2010). The impact of gender roles on disease expression, QoL and what it means to be a woman living with PD also deserves further inquiry so that intervention studies can be applied appropriately (Goodwin et al., 2008; Schrag, Jahanshahi, \& Quinn, 2000). 


\section{Chapter 3: Parkinson's Disease and Sex Differences in Electromyography During Daily}

\section{Life}

\subsection{Overview}

Parkinson's disease (PD) is a debilitating disease that affects control of muscular movement, which inevitably results in functional decline. PD-associated declines in physical function may be attributed to disruption of dopamine-regulated neural transmission within the substantia nigra of the basal ganglia, which can alter muscle activation patterns (DeLong \& Wichman, 2010; Galvan \& Wichman, 2008; Salenius et al., 2002). Muscle activation patterns are typically measured in laboratory environments through electromyography (EMG). EMG reveals important information about changes in neuromuscular activation that may directly limit strength, power and functional performance (Clark et al., 2011). Alterations in EMG patterns have been implicated in PD-related progressive motor dysfunction, such as slowness of movement (Pfann et al., 2001) and muscle weakness (Brown, Corcos, \& Rothwell, 1997; Stelmach, Teasdale, Phillips, \& Worringham, 1989; Logigian, Hefter, Reiners, \& Freund, 1991); however, the contributions of muscle activation and muscle weakness to functional decline in persons with PD has only been studied during controlled tasks. Evidence suggests that physical performance of persons with PD may vary with the task and environmental context (Teasdale, Phillips, \& Stelmach, 1990). Since environmental factors such as stress and fatigue fluctuate throughout the day and are not accounted for in controlled investigation (Christou, Jakobi, Critchlou, \& Enoka, 2004; Kent-Braun, 2009) it is imperative that measures of muscle activity be taken in the context of daily life.

Recordings of EMG within the environment where routine daily activities are performed have contributed valuable information to understanding patterns of muscle activation that allow classification of essential versus Parkinsonian tremor (Breit, Speiker, Schulz, \& Gasser, 2008). Muscle activation patterns also provide a measure of physical capacity required for workplace 
tasks and daily life in young and old adults (Harwood, Edwards \& Jakobi, 2011; Kern, Semmler \& Enoka, 2001), persons post-stroke (Jakobi, Edwards \& Connelly, 2008), frail females (Theou et al., 2010), persons with osteoarthritis (Howe \& Rafferty, 2009), and retirement dwelling older females (Theou, Bruce, Roland, Jones, \& Jakobi, 2011a). Quantification of muscle activation for a typical day through periods of bursts as well as gaps in EMG offers a means to understand neural alterations associated with aging, physical function and sex differences (Clark, Patten, Reid, Carabello, Phillips \& Fielding, 2010; Clark et al., 2011; Harwood et al., 2011). Specifically, the assessment of muscle quiescence through the quantification of gaps in the EMG signal has indicated that upper and lower leg muscles experience fewer periods of quiescence in older compared with younger adults, as well both young and old females compared with males (Harwood et al., 2011). Differences in sex-specific risk and clinical presentation are evident in PD (Ben Shlomo, 1997; Baba et al., 2005; Haaxma et al., 2007; Miller \& Cronin-Golomb, 2010; Roland, Jakobi, Powell, \& Jones, 2011b; Rubin, 2007; Tsang et al., 2001; Twelves et al., 2003) and females with PD experience greater declines in muscle strength, power, and force compared with males (Marusiaka, Jaskolska, Kisiel-Sajewicz, Yue, \& Jakolski, 2009; Pfann et al. 2001; Wierzbicka, Wiegner, Logigian, \& Young, 1991). Females with PD have more bursts, reach lower peak velocities, and lower magnitude agonist EMG than males with PD during isolated elbow flexion laboratory tasks (Pfann et al., 2001). Thus, it is important to consider sex differences in muscle activity patterns during daily life to better understand how these differences affect physical function necessary for independent living between males and females.

Declines in muscle strength and power impact one’s physical function required to engage in independent living (Jankovic \& Kapadia, 2001). Reduced lower-extremity function is a predictor of physical performance, functional mobility, and onset of disability in older adults (Guralnik, Ferrucci, Simonsick, Salive, \& Wallace, 1995; Guralnik et al., 2000). Although grip strength is frequently used as an overall measure of strength and lower extremity function 
(Berkman, Seeman, \& Albert, 1993; Fried et al., 1994; Hirsch, Fried, Harris, Fitzpatrick, Enright, \& Schulz, 1997) it cannot assess the contribution of individual muscles to daily movement that is executed bilaterally. Assessment of bilateral muscle activation of the upper and lower body is essential, as the influence of dopaminergic degeneration associated with PD progression may be unequal between sides (Hallett \& Koshbin, 1980; Kakinuma et al., 1998; Nogaki et al., 1995). Researchers report bilateral asymmetric muscle weakness, where the more affected PD side is typically weaker and experiences greater muscular fatigue (Koller \& Kase, 1986; Djaldetti et al., 2006). This highlights the need to investigate whether asymmetrical patterns of muscle activation exist in persons with PD as they go about normal daily activities and whether this contributes to muscle weakness, which may lead to functional decline.

The purposes of this investigation were to: 1) Quantify daily muscle activation during independent living; specifically, determine patterns of muscle quiescence through gaps in the EMG signal; and 2) Examine muscle strength in males and females with PD. It is hypothesized that muscle quiescence will be less in persons with PD compared to age-matched controls and that fewer gaps will be evident in the EMG signal of PD females compared with PD males. The side of the body most affected by PD will demonstrate fewer EMG gaps and greater muscle weakness relative to the less affected side.

\subsection{Methods}

\subsubsection{Research Design}

The Clinical Research Ethics Board of the University of British Columbia granted ethical approval and subjects provided written informed consent prior to participation. Males and females living independently at home with mild to moderate PD severity (stages I-III, Hoehn \& Yahr, 1967) were recruited from the local community. Control subjects were recruited from the same local community to match for age and sex within similar cohorts of persons with PD. For 
example, if there were three female subjects with PD of 61 years, one sex- and age-matched control was recruited. All subjects were right-hand dominant, independently mobile (with or without gait-aids), cognitively sound, and considered to be in a steady clinical state (i.e., controlled by medication). A disease history questionnaire was used to gauge disease duration, carbidopa-levodopa regime, and self-reported more affected body side.

Muscle quiescence was recorded over the course of a typical day with a portable EMG device (Biometrics DataLOG, Gwent, UK) (9.5x15.8x3.3 cm; 380 g) secured to a belt worn on the left side of the waist. The experimental protocol has previously been outlined (Harwood et al., 2011; Jakobi et al., 2008; Theou et al., 2010; Theou et al., 2011a). Briefly, all subjects were visited in their home during the early morning, either one to two hours post anti-Parkinson's medication or following breakfast for control subjects. During the testing session, subjects completed questionnaires, executed maximal handgrip, were set-up with the EMG equipment and performed a maximal voluntary exertion (MVE) for each muscle group. Adherence to a typical daily routine was encouraged with the exception of activities that would dislodge the EMG electrodes or damage the recording device (water-based activities). Approximately seven hours later, the researcher returned to the subject's home to complete maximal handgrip and MVE, and then remove the equipment.

Bilateral recordings of EMG were made for biceps brachii (BB), triceps brachii (TB), vastus lateralis (VL), and biceps femoris (BF) for the duration of the day as well as for MVE. To determine maximal EMG to normalize the daily recordings, the subject sat in an arm-less chair with a sturdy back and the experimenter held the respective joint (elbow, knee) at 90-degrees. The subject was encouraged verbally to apply maximal force against the resistance of the researcher for approximately seven seconds. Failure to maintain consistent and proper position during the MVE warranted additional attempts. Each muscle was tested bilaterally three times with approximately 60 sec of rest in-between trials to prevent fatigue. MVE were repeated at 
end-of-day to verify the integrity of the recording. The muscle activation recordings from the MVE were used to normalize the daily EMG data. The normalized daily EMG recordings for the leg muscles (VL, BF) were compared between PD and controls, and the recordings of all muscles (BB, TB, VL, BF) were compared between self-reported more and less affected sides in persons with PD.

\subsubsection{Electromyography}

Surface EMG electrodes with bipolar differential sensors $(3.7 \mathrm{~cm}$ x $2.2 \mathrm{~cm})(\mathrm{SX} 230$, Biometrics Ltd, Gwent UK) and a 20 mm inter-electrode distance were placed on the muscles according to the SENIAM recommendations for the $\mathrm{BB}, \mathrm{TB}, \mathrm{VL}$ and $\mathrm{BF}$, as previously described in prior work from our laboratory (Harwood et al., 2011; Jakobi et al., 2008; Theou et al., 2010; Theou et al., 2011a). Signals were sampled at 1,000 Hz, amplified (1,000 x), band-pass filtered (20-450 Hz), and stored on a MMC flashcard for offline analysis. EMG was imported into Biometrics software (Biometrics DataLog v.3, Gwent, UK) for preliminary visual inspection and subsequently in 1ms increments into Spike 2 V.5 (Cambridge Electronics Design, UK) for analysis in custom script software. Data artefacts were identified by taking the root mean square (RMS) amplitude of the waveform in $0.1 \mathrm{sec}$ increments and isolating plateaus (square waves) in the recording, which were manually removed in a time-locked manner across all channels. Waveforms were rectified, smoothed in $0.01 \mathrm{sec}$ intervals and down-sampled by a factor of 100 (Figure 3.1.). The MVE value used to normalize the EMG recordings for each subject was the average amplitude of the processed waveform at the midpoint of the MVE. Muscle quiescence was defined as a period of inactivity less than 1\% amplitude of the MVE for a continuous duration longer than 0.1 sec (Harwood et al., 2011). The individual gaps were used to calculate the number of gaps (periods of quiescence), mean gap duration (individual duration of each period of quiescence), and gap percentage (percentage of total time occupied by gaps). 

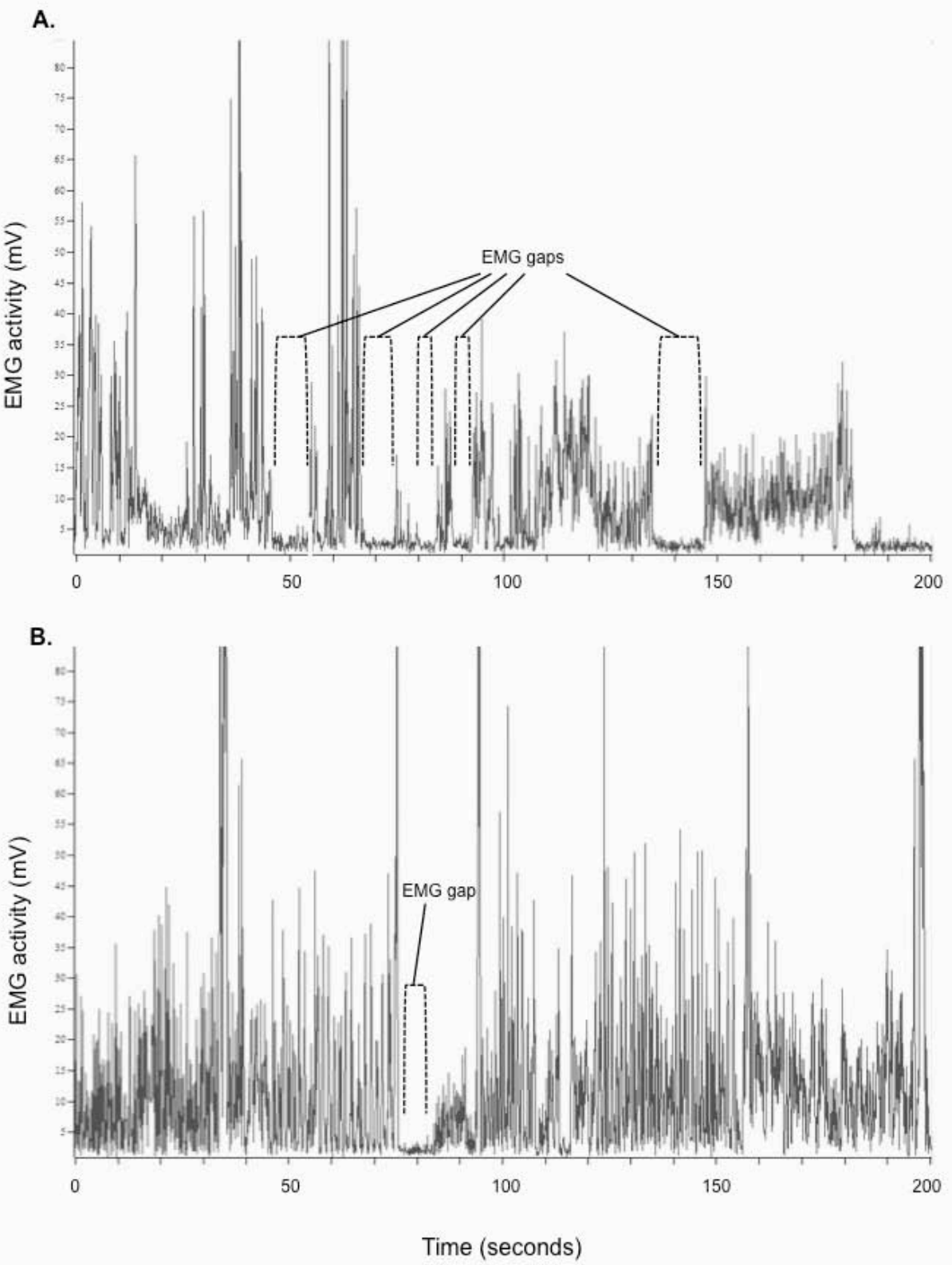

Figure 3.1. A Representative Electromyography Recording of Vastus Lateralis.

In a 54-year old female without PD (A) and a 55-year old female with PD (B) for a 200-second section of a daily EMG recording. EMG traces are rectified, smoothed (0.01s increments) and down sampled (100 x). EMG amplitude is truncated at $80 \mathrm{mV}$ to better visualize the gaps; however the average amplitude of both signals was similar (122mv). EMG, electromyography; mV, millivolt; PD, Parkinson’s disease. 


\subsubsection{Functional Activity}

Daily physical activity was measured using an ActiTrainer accelerometer (Actigraph, LLC, Fort Walton Beach, FL) worn on the waist on the right side. The ActiTrainer (8.6x3.3x1.5 cm; $51 \mathrm{~g}$ ) is a tri-axial solid-state accelerometer that was programmed to record 60 sec epochs of data. Prior to statistical analysis, ActiTrainer data was downloaded into the ActiLife software (Actigraph, LLC, Fort Walton Beach, FL). Number of steps per minute was recorded to ensure daily physical activity was comparable between subjects.

Maximal grip strength (kg) was evaluated in each hand individually using a handheld dynamometer (Almedic, St. Laurent, PQ). Subjects were asked to perform three trials bilaterally of maximal handgrip in the morning and end-of-day. Hands were alternated and subjects were allowed 60-120 sec rest between trials to prevent fatigue.

\subsubsection{Statistical Analysis}

Statistical Package for Social Sciences version 19.0 was used for analysis. Statistical significance was defined as $\mathrm{p}<0.05$. A paired t-test was used to compare morning and end-ofday MVE. The MVE recordings between the morning and end-of-day sessions did not differ $(\mathrm{p}=$ 0.67); therefore, the highest MVE regardless of time of day was used for normalization. A oneway analysis of variance (ANOVA) was performed to compare disease characteristics (i.e. severity, duration, carbidopa-levodopa dose) between males and females with PD. A two-way ANOVA was used to assess subject characteristics and daily physical activity (step count) between sexes (male, female) and disease (PD, control) (Table 3.1.). To evaluate the betweensubjects factors of sex (male, female) and disease (PD, control) and the within-subjects factor of muscle (VL, BF) for each of the dependent variables of gap number, gap duration, and gap percentage a 3-way repeated measure ANOVA was executed. In subjects with PD, a 3-way repeated measures ANOVA was also used to evaluate the between-subjects factor of sex (male, female), and the within-subjects factors for side of the body (more affected, less affected) and 
muscle (BB, TB, VL, BF) for the three dependent variables of gap number, gap duration, and gap percentage. The maximal handgrip values between morning and end-of-day sessions did not differ $(\mathrm{p}=0.54)$, therefore the highest handgrip regardless of time of day, was evaluated using a 3-way repeated measures ANOVA for sex (male, female) x disease (PD, control) x side (more affected, less affected). The control subjects, matched for age and sex, were also matched for the affected side. For example, if a subject with PD self-reported 'right' as the most affected side then the right side was also considered as 'more affected' for the age and sex-matched control. Probability level was set at $\mathrm{p}<0.05$ and significant interactions and main effects were assessed with Tukey post hoc and Independent t-tests. Data in text are reported as values \pm standard deviation of the mean and figures are reported as values \pm standard error of the mean (SEM).

\subsection{Results}

\subsubsection{Subject Characteristics}

Thirteen females and 10 males with PD (mean age $=67 \pm 7.7$ years) and eight females and six male controls (mean age $=66 \pm 9.2$ years) were investigated. In subjects with PD, disease duration ranged from one to 16 years (mean $=8 \pm 5.8$ years) and average disease severity was $2.1 \pm 0.7$ (Hoehn \& Yahr score). Disease duration, severity and carbidopa-levodopa dose were similar between males and females with PD. Age, body mass index, and daily step count did not differ between groups ( $\mathrm{p}>0.2$, Table 3.1.). 
Table 3.1. Characteristics of Males and Females with Parkinson's Disease and Controls

\begin{tabular}{|c|c|c|c|c|}
\hline & \multicolumn{2}{|c|}{ Control } & \multicolumn{2}{|c|}{ PD } \\
\hline & $\mathbf{M}$ & $\mathbf{F}$ & $\mathbf{M}$ & $\mathbf{F}$ \\
\hline Number & 6 & 8 & 10 & 13 \\
\hline Age (years) & $68 \pm 9$ & $65 \pm 9$ & $67 \pm 7$ & $67 \pm 8$ \\
\hline BMI (m/kg) & $26.7 \pm 4$ & $25.0 \pm 5.3$ & $24.9 \pm 2.7$ & $23.5 \pm 4.1$ \\
\hline Daily step count & $2705.0 \pm 1968.3$ & $3599.9 \pm 2425.6$ & $3554.5 \pm 2894.8$ & $3265.2 \pm 3065.8$ \\
\hline HY & & & $2.1 \pm 0.8$ & $2.2 \pm 0.7$ \\
\hline PD duration (years) & & & $7.2 \pm 4.0$ & $8.8 \pm 8.8$ \\
\hline CL (mg) & & & $625.0 \pm 574.1$ & $623.1 \pm 329.5$ \\
\hline R dominant, R PD & 3 & 5 & 5 & 7 \\
\hline R dominant, L PD & 3 & 3 & 5 & 6 \\
\hline
\end{tabular}

\subsubsection{Daily Muscle Quiescence}

The sex-disease-muscle interaction was non-significant for all gap characteristics ( $\mathrm{p}>$ 0.23). All interactions and main effects were non-significant for number of gaps ( $p>0.23$;

Figure 3.2.A). The sex by disease and sex by muscle interactions were non-significant for average gap duration and percentage of gaps $(\mathrm{p}>0.1)$. The disease by muscle interaction for gap duration was non-significant $(p=0.99)$. Main effects were observed for $\operatorname{sex}(F(1,31)=9.48, p=$ $0.004)$ and disease $(F(1,31)=5.98, p=0.04)$, where the duration of the gaps were $65 \%$ shorter in females and 60\% shorter in persons with PD than males and control subjects, respectively (Figure 3.2.B). For percentage of gaps there was a significant interaction for disease by muscle $(\mathrm{F}(1,31)=5.89, \mathrm{p}=0.02)$ as the $\mathrm{VL}$ had $39 \%$ more gaps than $\mathrm{BF}$ in persons without $\mathrm{PD}(\mathrm{F}(1,10)$ $=10.703, p=0.01)($ Figure 3.2.C). A main effect was observed for $\operatorname{sex}(F(1,31)=5.35, p=0.03)$, where gaps occupied 56\% less time in females compared with males in persons with PD. A main 
effect was observed for disease $(F(1,31)=6.02$, $p=0.02)$, where gaps occupied half (i.e., $100 \%$ less) the amount of time compared with controls.

The sex-side-muscle interaction was non-significant for all gap characteristics ( $\mathrm{p}>0.80$ ). The sex by side, sex by muscle and side by muscle interactions were non-significant for number of gaps, gap duration and percentage of gaps ( $p>0.40)$. Females had 22\% fewer gaps compared to males $(\mathrm{F}(1,17)=4.59, \mathrm{p}=0.04)$ and the more affected side had $19 \%$ fewer gaps than the less affected side $(\mathrm{F}(1,17)=4.66, \mathrm{p}=0.04)$ (Figure 3.3.A). The main effect of muscle was nonsignificant for number of gaps $(p=0.20)$. Duration of gaps were $66 \%$ shorter in females compared with males $(F(1,17)=6.68, p=0.02)$ (Figure 3.3.B). Main effects of side $(p=0.91)$ and muscle $(\mathrm{p}=0.65)$ were non-significant for average gap duration. Percentage of gaps was $51 \%$ lower in females compared with males $(\mathrm{F}(1,17)=4.91, \mathrm{p}=0.04)$ and $15 \%$ lower on the more affected than less affected PD side $(F(1,17)=8.24, p=0.01)$ (Figure 3.3.C). The main effect of muscle $(F(1,17)=5.27, p=0.03)$ was driven by higher percentage of EMG activity in the BB and TB compared with VL and BF ( $\mathrm{p}=0.00-0.046)$ (Figure 3.3.D).

The sex-disease-side interaction was non-significant $(\mathrm{p}=0.78)$. The sex by disease and sex by side interactions were non-significant $(\mathrm{p}>0.41)$. The side by disease interaction was significant $(F(1,74)=6.14, p=0.02)$ and was driven by the strength differences between more affected side in persons with PD compared with matched side in controls (Table 3.2). Strength was similar between persons with PD and controls on the less affected side $(p=0.20)$; however, persons without PD were 20\% stronger than persons with PD on the matched more affected side $(p=0.006)$. Main effects of sex, disease and side were demonstrated. Regardless of disease diagnosis, males were $40 \%$ stronger than females $(F(1,74)=67.59, p=0.00)$, persons with PD were $15 \%$ weaker than controls $(F(1,74)=3.7, \mathrm{p}=0.05)$ and the less affected side was $17 \%$ stronger than the more affected side in all subjects $(F(1,74)=3.8, p=0.05)$. 
A.

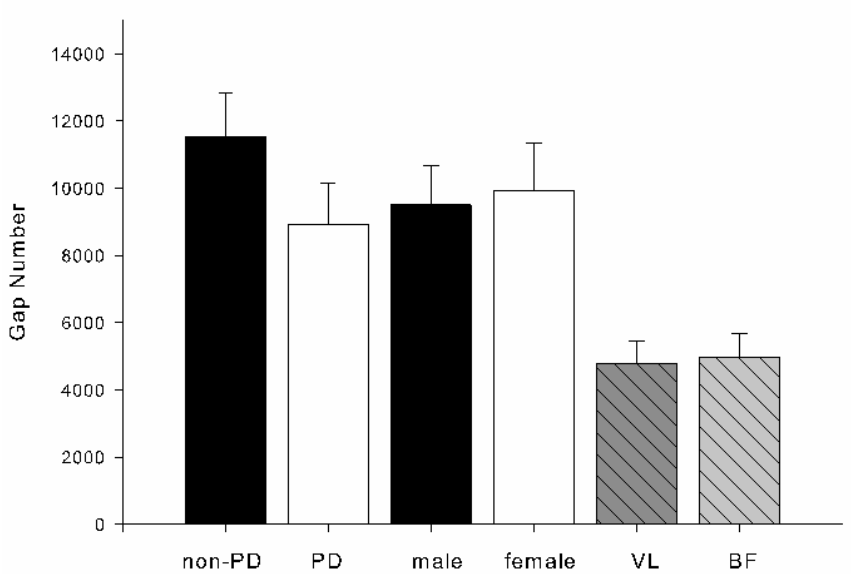

B.

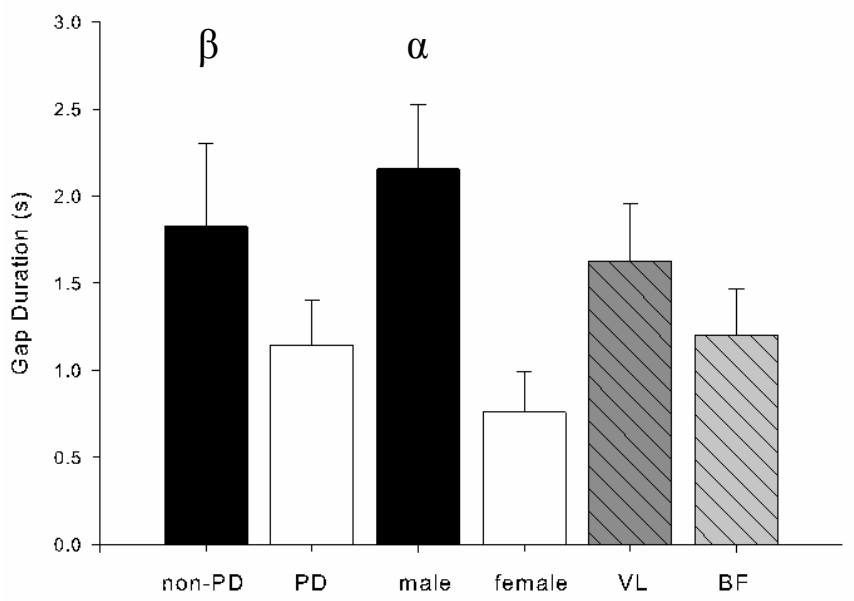

C.

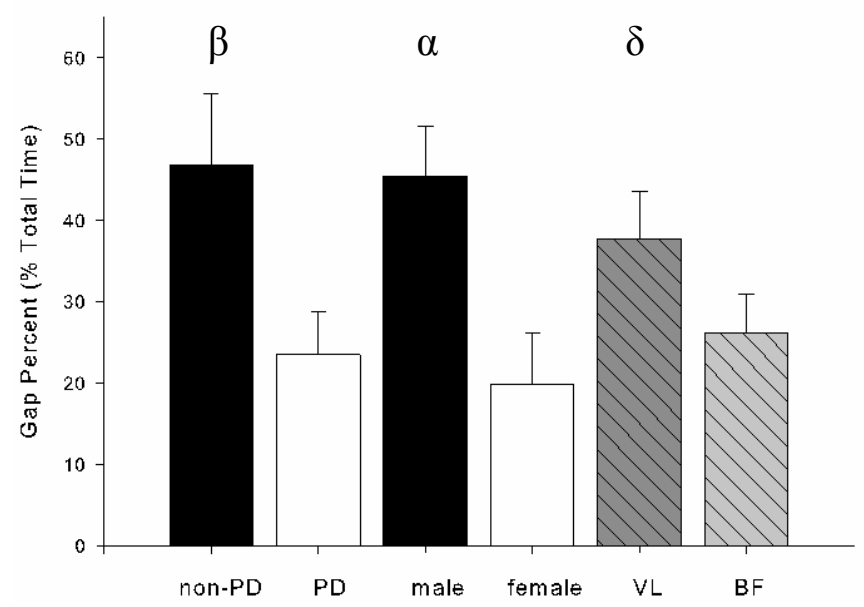

Figure 3.2. Disease and Sex Differences in Gap Characteristics.

Number of gaps (A), average gap duration (B), gap percent (C) for VL (dark gray hatched bar) and BF (light gray hatched bar) in males (black bar) and females (white bar) with (black bar) and without (white bar) PD. $\alpha$, significantly different from females; $\beta$, significantly different from PD; $\delta$, significantly different from BF. PD, Parkinson’s disease; VL, vastus lateralis; BF, biceps femoris. Data is expressed as means \pm SEM. 
A.

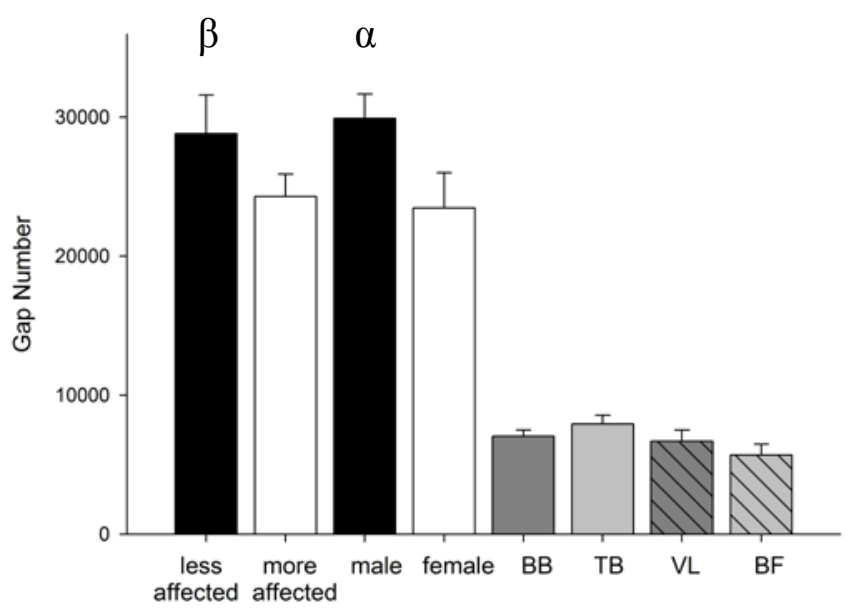

B.

C.
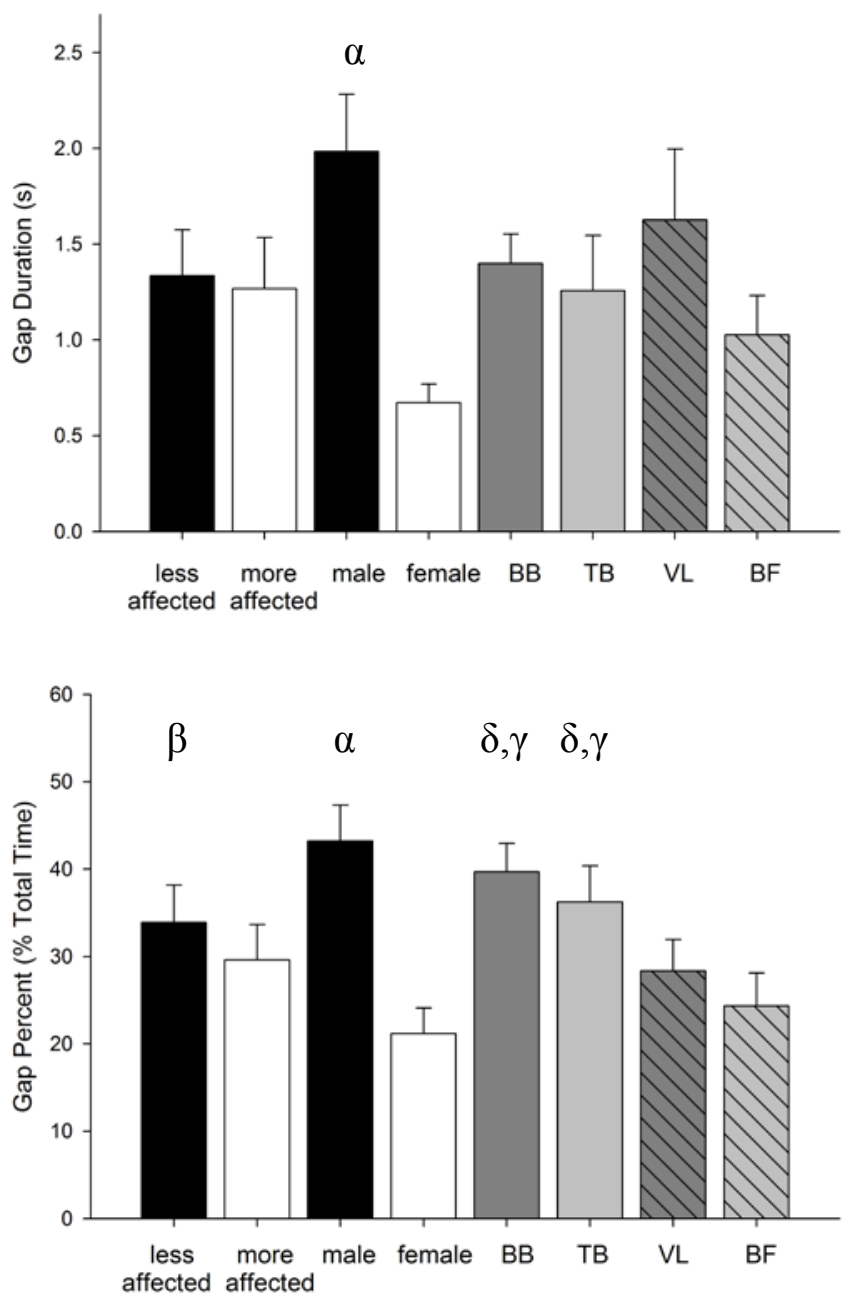

Figure 3.3. Side and Sex Differences in Gap Characteristics

Number of gaps (A), average gap duration (B), and gap percent (C) in males (black bar) and females (white bar) with PD on less affected (black bar) and more affected (white bar) side for the muscle groups BB (dark gray bar), TB (light gray bar), VL (dark gray hatched bar) and BF (light gray hatched bar). $\alpha$, significantly different from females; $\beta$, significantly different from more affected PD side; $\delta$, significantly different from VL; $\gamma$, significantly difference from BF. BB, biceps femoris; BF, biceps femoris; PD, Parkinson's disease; TB, triceps brachii; VL, vastus lateralis. Data is expressed as means \pm SEM. 
Table 3.2. Handgrip Strength

\begin{tabular}{lccccccc}
\hline & All & & M & F & & Control & PD \\
\cline { 2 - 2 } Both sides & $32.3 \pm 1.0$ & & $40.6 \pm 1.4^{\beta}$ & $24.2 \pm 1.4$ & & $35.2 \pm 1.6^{\mathrm{\gamma}}$ & $30.0 \pm 1.1$ \\
More affected side & $31.7 \pm 1.03^{\alpha}$ & & $40.9 \pm 1.4$ & $24.7 \pm 1.4$ & & $37.7 \pm 11.4^{*}$ & $31.4 \pm 9.4$ \\
Less affected side & $38.2 \pm 1.00$ & & $39.8 \pm 1.5$ & $23.8 \pm 1.5$ & & $36.2 \pm 9.9$ & $34.2 \pm 10.4$
\end{tabular}

Data is reported in kilograms and expressed as mean \pm standard deviation; M, male; F, female; PD, Parkinson's disease; ${ }^{\alpha}$ Significantly different from All less affected side $(\mathrm{p}=0.05) ;{ }^{\beta}$ Significantly different from $\mathrm{F}$ both sides ( $\mathrm{p}$ $=0.00) ;{ }^{\vee}$ Significantly different from PD both sides $(\mathrm{p}=0.05) ;{ }^{*}$ Significantly different from PD more affected side $(\mathrm{p}=0.006)$

\subsection{Discussion}

This is the first study to examine patterns of daily muscle quiescence, by quantifying gaps in the EMG signal, in persons with PD during independent daily living with consideration of the potential for sex differences. In persons with PD, duration of gaps was shorter and gaps occupied less total time in the EMG signal than controls. Also, there were fewer number of gaps with shorter duration, resulting in less total time occupied by gaps as determined by the EMG signal in females compared to males with PD. On the more affected side the number of gaps were fewer compared to the less affected side of persons with PD. Muscle strength in persons with PD was less than controls and females were weaker than males. In persons with PD the more affected PD side was weaker than the less affected side. This study is important because it reports that muscle quiescence is decreased in persons with PD and this reduction is greater in females and on the more affected side. This is evidence that females with PD experience greater declines in neuromuscular capacity than males, and that asymmetry in muscle activation and strength may indicate differential disease progression between males and females. 


\subsubsection{Disease-Related Differences}

Previous investigations of muscle activity during controlled laboratory-based tasks of elbow flexion have demonstrated that persons with PD have greater number of bursts, and decreased duration of agonist bursts compared with controls (Pfann et al., 2001; Robichaud et al., 2002). The results of this investigation indicate that the percentage of time leg muscles are quiescent is decreased in persons with PD because of a shorter duration of each EMG gap. The leg muscles were compared because lower-extremity function predicts the subsequent development of disability, and leg function may reflect the effects of chronic disease, coexisting conditions, and physiologic decline that have not yet caused dependent living (Guralnik et al., 1995; Guralnik et al., 2000). Dopamine degeneration leading to impaired variability, intensity and frequency of corticospinal activation result in neuromuscular changes associated with PD (David et al., 2012). These central changes influence motor nerve firing patterns and may contribute to decreased muscle quiescence (DeLong \& Wichman, 2010; Galvan \& Wichman, 2008; Miller, Thaut, McIntosh, \& Rice, 1996). A reduction in muscle quiescence in persons with PD compared with controls during independent daily living in the present study support previous findings that greater numbers of bursts and more overall muscle activity is necessary to complete individual tasks (Hallet \& Koshbin, 1980; Teasdale, Phillips, \& Stelmach, 1990). Dopaminergicrelated declines in physiological capacity combined with presentation of PD symptom makes it challenging for persons with PD to complete routine daily activities. It is possible that clinical symptoms (i.e., rigidity, tremor, dyskinesias) may have an impact on muscle activation patterns; however, the presence of dyskinesias and rigidity were very low in these subjects. Previous evidence suggests medication improves motor function, movement speed, and EMG magnitude of the first agonist burst (Robichaud et al., 2002), while medication withdrawal results in further weakness (Corcos, Chen, Quinn, McAuley, \& Rothwell, 1996). Persons with PD were examined on their typical medication protocols over an entire day; therefore, future research is necessary to 
fully understand the influence of dopaminergic dose-response cycling and symptom fluctuations on muscle activation patterns during the course of a day.

Handgrip strength is a reliable indicator of physical function in persons with PD (Jordan et al., 1992). Degeneration of dopamine neurons in PD is primarily seen in the substantia nigra pars compacta and ventral tegmental area, and secondarily in the striatum and motor areas of the cerebral cortex (Gaspar et al., 1991). In this study, 15\% decrease in handgrip strength in persons with PD may reflect the inability of the motor cortex to activate spinal motor neurons in PD (Salenius et al., 2002). Muscular weakness may also result from slowed onset of voluntary contractions characteristic of PD, thus taking longer to develop peak grip force compared with controls (Brown et al., 1997; Brown, Corcos, \& Rothwell, 1998; Brown, 2003; Fellows et al., 1998; Salenius et al., 2002). As well, peripheral changes such as increased type-I and decreased type-II fibers of the biceps brachii in PD (Edstrom, 1970) also likely contribute to muscle weakness.

\subsubsection{Sex-Related Differences}

There was a reduction in muscle quiescence in females compared with males during independent daily living. Previous studies investigating muscle activation patterns indicate that females with PD have more muscle activity, reach lower peak velocities, and have a lower magnitude agonist EMG than males with PD during isolated elbow flexion (Pfann et al., 2001). Other investigations in the general population report shorter gap duration and decreased number of gaps in females compared with males during four to eight hours of normal daily living (Harwood et al., 2011; Nordander et al., 2008). This is the first investigation to demonstrate sexrelated differences in patterns of muscle quiescence collected as the person with PD went about their routine daily life activities. Fewer periods of muscle quiescence in females compared to males with PD indicates that muscles have less opportunity to recover (Blangsted, Hansen, \& Jensen, 2003; Laursen, Jensen, \& Ratkevicius, 2001; Harwood, Edwards, \& Jakobi, 2008), and 
suggests that a greater level of muscle activation is necessary for independent living. This sexrelated decrease in muscle quiescence, or recovery time, may contribute to greater muscular fatigue (Harwood et al., 2008) leading to greater declines in physical function seen in females with PD compared with males (Ben Shlomo, 1997; Haxma et al., 2007; Miller \& CroninGolomb, 2010; Roland et al., 2011b).

Females with PD experience greater declines in muscle strength and power compared with males (Marusiaka et al., 2008; Pfann et al., 2001; Wierzbicka et al., 1991). The results of this study demonstrate handgrip strength was less in females compared with males and this may be explained by loss of muscle mass. Sarcopenia, defined as the age-related loss of skeletal muscle mass, is a greater problem for females since they have significantly less initial muscle mass and live longer with higher rates of disability compared with males (Doherty, 2001). In PD, females demonstrate lower levels of fat-free mass compared with sex- and age-matched controls during menopausal transition, which suggests accelerated sarcopenia with PD (Poehlman et al., 1995).

\subsubsection{Sidedness}

The present study reports fewer gaps during normal daily activities on the more affected PD compared with less affected side. Also, the more affected side was weaker than the less affected side in persons with PD compared with controls. Reported asymmetry in muscle activation and strength in PD may provide an indication of disease progression (Kakinuma et al., 1998; Nogaki et al., 1995). Specifically, the basal ganglia are somatotopically organized (Hallett \& Koshbin, 1980); therefore, it may be possible for one side of the body to be more affected while another is less affected. Perception of muscular force is mediated by the basal ganglia. Our results present differences in handgrip strength and daily muscle quiescence between more and less affected sides in persons with PD compared with controls. It can be speculated that decreased strength may result from inability to accurately perceive the muscular force exerted by 
their more affected hand (Lafargue, D’Amico, Thobois, Broussolle, \& Sirigu, 2008). Therefore, persons with PD may present with deficits in their physiological capacity and control necessary to successfully complete motor tasks during independent daily living (Lafargue et al., 2008).

\subsection{Final Remarks}

In persons with $\mathrm{PD}$, understanding and defining physiological change may help to prolong function, despite disease progression. The present study provides insight into mechanisms underlying muscle activation, which drives functional performance in males and females with PD as they go about normal daily activities. Overall, these findings suggest that PD- and sex-related differences in patterns of muscle quiescence likely reflect central and peripheral changes associated with dopamine that may explain muscular weakness. Further investigation is necessary to elucidate the contribution of sex-related differences in muscle activation and strength as a possible predisposing factor to the greater impact on physical function in females compared with males. Understanding neurophysiological parameters with respect to normal daily life using EMG will provide an indication of the underlying neuromuscular contribution to PD motor symptoms that influence daily life. 


\section{Chapter 4: Daily Electromyography in Females with Parkinson's Disease: A Potential Indicator of Frailty?}

\subsection{Overview}

Frailty is a complex geriatric syndrome that is clinically characterized by five criteria of weight loss, reduced endurance, slowed gait speed, muscle weakness and exhaustion, resulting in functional dependence (Fried et al., 2001). According to Fried and colleagues (2001), frailty can be assessed in terms of three phenotypes, or categories of frailty severity, which are defined by the sum of the five individual frailty criteria (i.e., 0: nonfrail, 1 or 2: prefrail, and 3-5: frail). Although frailty can exist independently, it may also co-exist in older adults with progressive neurological disorders, such as PD (Ahmed et al., 2008; Lauretani, Maggio, Silvestrini, Nardelli, Saccavini, \& Paolo Ceda, 2012; Powell, 2008; Roland, Jakobi, Powell, \& Jones, 2012). Comorbidities and clinical symptoms interact between frailty and PD making identification of frailty in persons with PD challenging to diagnose (Powell, 2008). Few studies have examined how to identify frailty in the PD population (Ahmed et al., 2008; Lauretani et al., 2012).

Electromyography (EMG) recordings provide a measure of muscle activity that governs movement associated with sustaining physical function. EMG detects the bioelectrical activity associated with muscle contractions that produce movements and reveals important neural adaptations associated with aging and decline in physical function (Clark et al., 2010; Clark, 2011). Portable EMG devices have proven to be a viable means to record muscle activity in older adults during daily life and offer a method to detect frailty-, disease- and age-related differences in muscle activity required to execute movement (Harwood et al., 2011; Howe \& Rafferty, 2009; Jakobi et al., 2008; Kern et al., 2001; Theou et al., 2010; Theou et al., 2011a).

Recent investigations from our laboratory (Theou et al., 2010; Theou et al., 2011a) have used EMG to quantify periods of muscle quiescence (gaps) and muscle activity (bursts) in frail older females as they went about their routine daily activities. As the level of frailty advanced 
the number of gaps increased while the duration of gaps decreased, and the number of bursts decreased while the burst duration increased (Theou et al., 2010). This suggests that EMG can detect differences between frailty phenotypes. EMG recordings of daily muscle activation patterns that disassociate stages of frailty have not yet been quantified in females with PD.

The aim of this study was to determine whether daily muscle activation differs between frailty phenotype classifications in females with PD. This will facilitate identification of frailty in females with PD. It is hypothesized that greater periods of muscle quiescence and shorter periods of muscle activity will occur and this change will be exacerbated as females with PD progress across frailty phenotypes. These changes in muscle characteristics will be observed, despite the presence of PD. Understanding how muscle activity changes across frailty phenotypes may help to inform interventions aimed at delaying frailty progression in females with PD. Application of this new knowledge may improve practice by providing an indicator of changes in muscle activity that precipitates increased disability in PD.

\subsection{Methods}

\subsubsection{Study Population and Design}

Females diagnosed with mild to moderate PD (stages I-III; Hoehn \& Yahr, 1967) living in their own home were recruited through newsletter advertisement, public presentations and word-of-mouth. Interested females from the Greater Vancouver and Okanagan areas of British Columbia contacted a member of the research team to participate between March 2010 and January 2012. Subjects were right-hand dominant, able to ambulate independently (with or without gait-aid), cognitively sound, and in a steady clinical state (i.e., controlled by medication). The Clinical Research Ethics Board of the University of British Columbia granted ethical approval for this investigation and subjects provided written informed consent. Continuous EMG was collected for approximately six and a half hours on one testing weekday within the subjects' 
personal living environment (i.e., home, neighbourhood). Health history questionnaire (including disease characteristics), frailty assessment and set-up of portable EMG equipment occurred one to two hours post morning anti-Parkinson's medication. The researcher returned approximately six and a half hours later to the subject's home to remove the equipment. Maximal voluntary exertions (MVE) for each muscle group were executed in the morning and at the end-of-day to verify the signal integrity, as well as normalize the daily EMG recording to maximal. MVE consisted of an isometric contraction against manual resistance of the researcher. The subject sat with the joint (i.e., elbow or knee) at 90-degrees. The MVE for each muscle group was tested three times with approximately one minute of rest between trials to prevent fatigue. The MVE amplitude of the processed waveform at the midpoint for each of the four muscle groups was recorded. The greatest MVE of all trials, regardless of time of day, was used for normalization. During the testing day, subjects were encouraged to go about typical activities, but avoid strenuous or water-based activities that would affect the recording equipment.

\subsubsection{Electromyography Measurement}

Continuous surface EMG was collected over a six to seven hour day as subjects went about their routine daily activities. Surface EMG from the biceps brachii (BB), triceps brachii (TB), vastus lateralis (VL), and biceps femoris (BF) was recorded using a portable EMG device (Biometrics DataLOG P3X8, Gwent, UK). The hair was shaved (if necessary), and skin was abraded and cleaned with alcohol to reduce signal impedance. Surface electrodes with bipolar differential sensors (SX230, Biometrics Ltd, Gwent UK; 20 mm inter-electrode distance) were placed mid-belly on the muscle on the self-reported less affected side and a common ground electrode on the lateral malleolus of the left fibula, as previously described (Harwood et al., 2011; Jakobi et al., 2008; Theou et al., 2010; Theou et al., 2011a). The EMG data logger $(9.5 \times 15.8 \times 3.3 \mathrm{~cm} ; 380 \mathrm{~g})$ was secured to the left side of a belt worn at the waist. The EMG 
signals were sampled at 1,000 Hz, amplified (1,000 x), band-pass filtered (20-450 Hz), and stored on a MMC flashcard for offline analysis.

\subsubsection{Electromyography Analysis}

Continuous waveforms from the EMG signals were imported into Biometrics software (Biometrics DataLog v.3, Gwent, UK) for preliminary visual inspection and exported into Spike 2 V.5 (Cambridge Electronics Design, Cambridge, UK). Custom-script analysis was utilized to identify periods of muscle quiescence (gap) and muscle activity (burst) as subjects went about their daily routine. Artefacts in the EMG signal were identified through visual inspection of the data by isolating plateaus in the root mean square amplitude of the waveform and then manually removed in a time-locked manner across all channels, as previously described (Harwood et al., 2011; Jakobi et al., 2008; Theou et al., 2010; Theou et al., 2011a). Signal waveforms were rectified, smoothed at a time constant of 0.01 sec intervals and down-sampled by a factor of 100 (Figure 4.1.).

Muscle quiescence (gap) and activity (burst) has previously been employed to determine individual muscle activation over long durations (Harwood et al., 2011; Howe \& Rafferty, 2009; Jakobi et al., 2008; Kern, Semmler \& Enoka, 2001; Theou et al., 2010; Theou et al., 2011a). In accordance with this previous research, muscle quiescence was represented by a gap in the EMG signal. Gaps were quantified as EMG periods less than 1\% of MVE with the continuous EMG period greater than 0.1 seconds. Gap characteristics examined included; number of gaps, mean duration of each individual gap (seconds), and percentage of total recording time occupied by gaps (gap percent). A burst was defined as muscle activity greater than $2 \%$ of MVE with the continuous EMG period greater than 0.1 sec in duration. Burst characteristics examined included; number of bursts, mean duration of each individual burst (seconds), average peak amplitude of all bursts (\% MVE), and percentage of total time occupied by bursts (burst percent). 


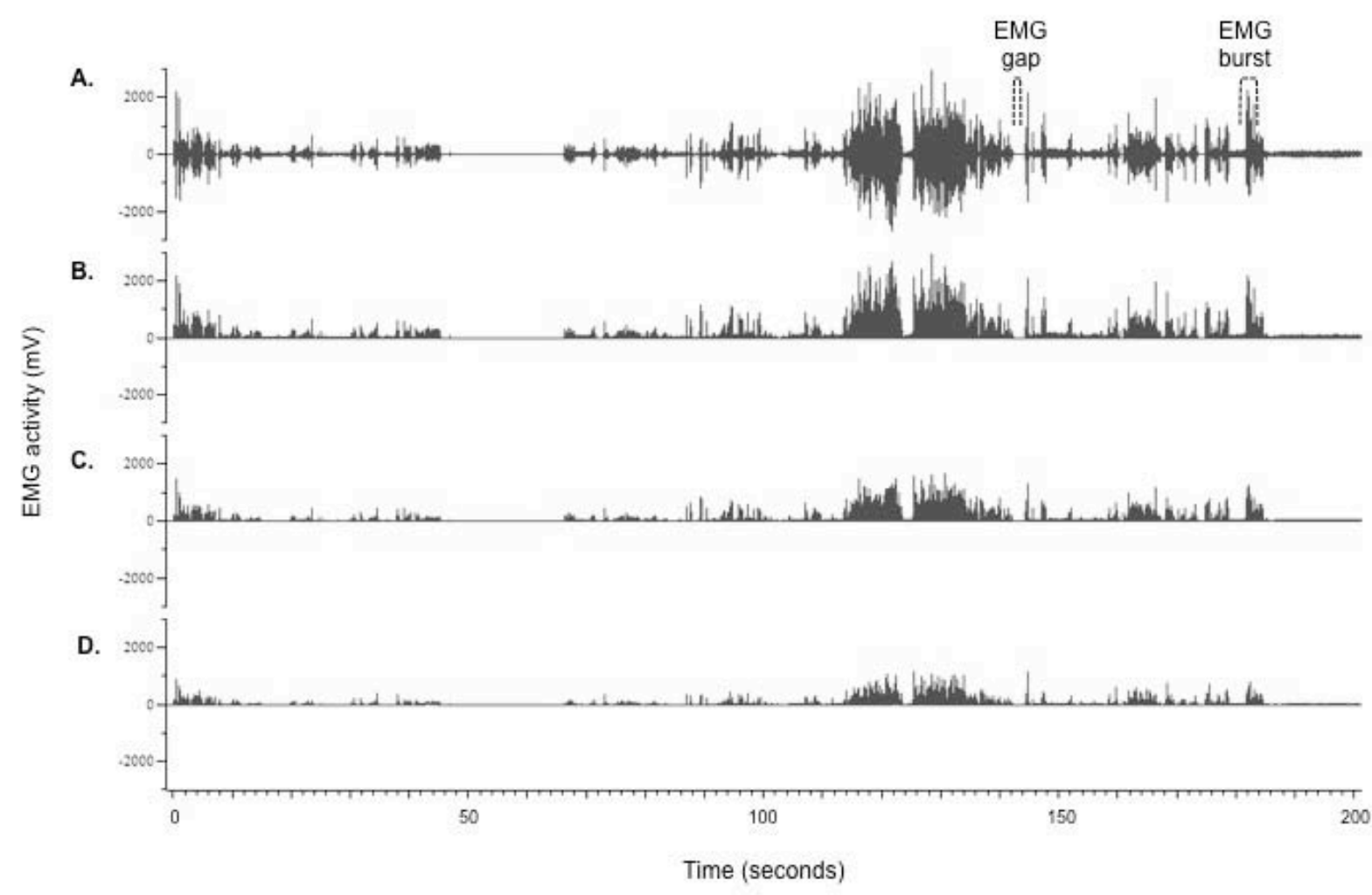

Figure 4.1. Electromyography Signal Processing.

A representative EMG recording from the less affected BF (left) of a 67-year old frail female with PD for a 200second section of daily activity. Original EMG traces (A) are rectified (B), smoothed (C), and down sampled (D). BF, biceps femoris; EMG, electromyography; mV, millivolt; PD, Parkinson’s disease.

\subsubsection{Frailty}

The Cardiovascular Health Study frailty index (CHSfi) assessment of physical frailty was administered. The CHSfi assesses five physical criteria to determine frailty phenotype classification, including; weight loss of greater than 10 lbs in the past 12 months, maximal handgrip strength, time to walk 15-ft at usual pace, self-reported leisure time physical activity, and self-reported exhaustion as described by the phenotype (Fried et al., 2001). Frailty phenotype was defined according to the number of criteria that the subject met. The nonfrail phenotype is defined as meeting none of the criteria, one - two criteria is scored as prefrail phenotype and three or more is defined as frail phenotype (Fried et al., 2001). 


\subsubsection{Physical Activity}

Daily physical activity was measured to ensure each frailty group was participating in similar activity levels throughout the testing day. A tri-axial solid-state waist-born accelerometer was used to measure individual daily step counts (Actigraph, LLC, Fort Walton Beach, FL). The Actitrainer accelerometer (8.6x3.3x1.5 cm; $51 \mathrm{~g})$ was worn for then entire testing day on the right hip and was programmed to record 60-sec epochs of data. The data was downloaded into the ActiLife software (Actigraph, LLC, Fort Walton Beach, FL) and exported into Microsoft Excel spreadsheet for subsequent analysis. Individual daily step counts were averaged for each frailty phenotype.

\subsubsection{Statistical Analysis}

Subject characteristics and daily physical activity were compared between frailty phenotypes using a one-way analysis of variance (ANOVA). To evaluate the between-subjects factor of frailty (nonfrail, prefrail, frail) and the within-subjects factor of muscle (BB, TB, VL, $\mathrm{BF})$, a 2-way repeated measures ANOVA was completed for each dependent variable of gap number, gap duration, gap percent, burst number, burst duration, burst amplitude, and burst percentage. Data was analyzed using the Statistical Package for Social Sciences 19.0 and statistical significance was defined as $\mathrm{p}<0.05$. Significant interactions and main effects were assessed with Tukey post hoc and pairwise comparisons. Data in text are reported as values \pm standard deviation of the mean and figures are reported as values \pm standard error of the mean. 


\subsection{Results}

\subsubsection{Subject Characteristics}

Thirteen community-dwelling females with PD (mean $=67 \pm 8$ years) participated and were classified as nonfrail $(n=4)$, prefrail $(n=6)$, and frail $(n=3)$ according to the CHSfi criteria. Power analysis determined a sample size of 13 subjects was sufficient $(>0.8)$ to detect a moderate effect size (cohen’s d = 0.5-0.7). All subject characteristics were similar between frailty groups ( $p=0.23-0.89)$, except Berg Balance Scale score was less in frail females compared with nonfrail $(\mathrm{p}=0.04)$ (Table 4.1).

Table 4.1. Characteristics of Females Across Frailty Phenotypes

\begin{tabular}{cccccc}
\hline & Total & & Nonfrail & Prefrail & Frail \\
\cline { 1 - 2 } Number & 13 & & & & \\
Mean age (years) & $67 \pm 8$ & & $69 \pm 1$ & $66 \pm 9$ & $67 \pm 9$ \\
BMI & $23.5 \pm 4.1$ & & $26.4 \pm 5.8$ & $22.3 \pm 3.9$ & $23.1 \pm 2.2$ \\
Number comorbidities & $1.1 \pm 1.3$ & & $1.7 \pm 1.2$ & $0.7 \pm 1.1$ & $0.7 \pm 1.2$ \\
Number of medication & $2.9 \pm 1.6$ & & $4.0 \pm 2$ & $2.9 \pm 1.5$ & $2 \pm 1$ \\
Disease severity (HY) & $2.1 \pm 0.6$ & & $1.8 \pm 0.8$ & $2.1 \pm 0.6$ & $2.3 \pm 0.3$ \\
Disease duration (years) & $8.6 \pm 7.1$ & & $3.3 \pm 2.3$ & $10.7 \pm 7.9$ & $5.7 \pm 1.2$ \\
Carbidopa-levodopa dosage (mg) & $623.1 \pm 329.5$ & $366.7 \pm 57.7$ & $700 \pm 387.3$ & $700 \pm 264.6$ \\
BBS & $22.5 \pm 2.7$ & $25.3 \pm 1.2 *$ & $22.3 \pm 1.8$ & $20.0 \pm 3.5$
\end{tabular}

* Significantly different from frail (p = 0.04) BMI, body mass index; HY, Hoehn \& Yahr disease severity; mg, miligrams; BBS, Berg Balance Scale 


\subsubsection{Electromyography}

\subsubsection{Gap Characteristics}

The frailty by muscle interaction was non-significant for each of the gap characteristics ( $p=0.52-0.88)$. The main effect of frailty for gap percent and gap number was significant. The percentage of time occupied by gaps was $59 \%$ less in frail females compared with nonfrail females $(F(2,30)=7.65, p=0.02)$ (Figure 4.2.A), and frail females had 73\% fewer gaps than nonfrail females $(F(2,30)=7.104, p=0.01)$ (Figure 4.2.B). The main effect of frailty $(p=0.93)$ was non-significant for average gap duration (Figure 4.2.C). The main effect of each muscle for all gap characteristics was non-significant $(\mathrm{p}=0.09-0.79)$.

\subsubsection{Burst Characteristics}

The frailty by muscle interaction for each burst characteristic was non-significant ( $\mathrm{p}=$ 0.24-0.63). Frail females had a 42\% greater percent of time occupied by bursts than nonfrail $(\mathrm{F}(2,30)=4.38, \mathrm{p}=0.04)$ (Figure 4.3.A). The main effect of frailty was non-significant for burst number $(p=0.88)$ (Figure 4.3.B). Frail females had a 48\% greater burst duration than nonfrail $(\mathrm{F}(2,30)=3.99, \mathrm{p}=0.04)$ (Figure 4.3.C). The main effect of frailty was non-significant for mean peak amplitude $(\mathrm{p}=0.50)$ (Figure 4.3.D). The main effect of muscle was non-significant for all burst characteristics $(p=0.10-0.24)$, except for burst amplitude $(F(3,30)=3.97, p=0.02)$ the TB had $45 \%$ less burst amplitude than that of BF $(p=0.03)$. 
A.

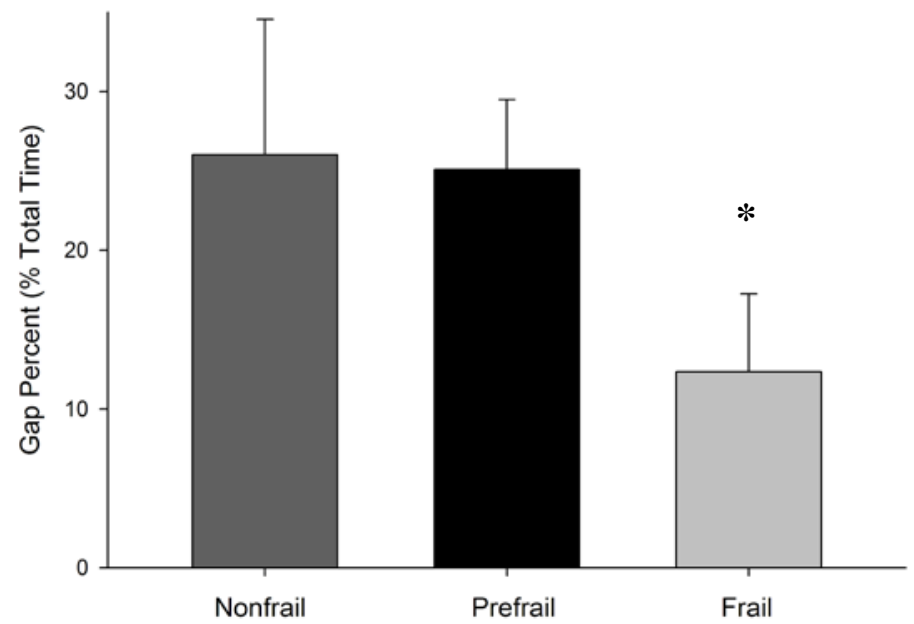

B.

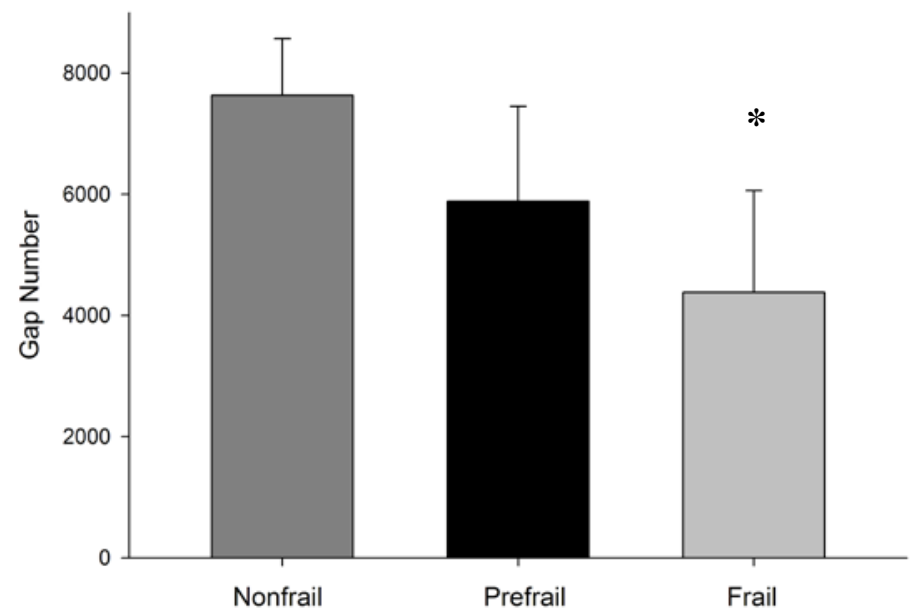

C.

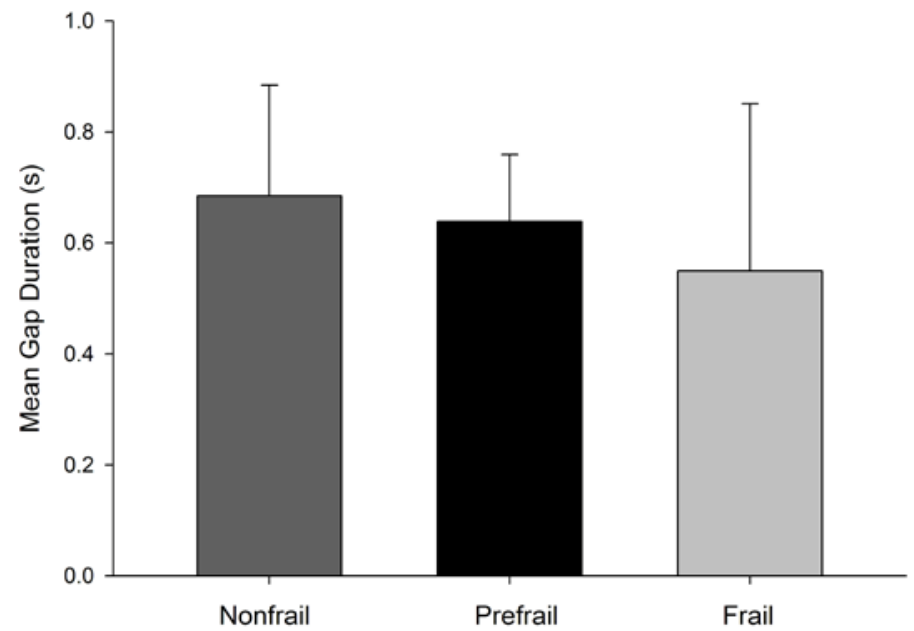

Figure 4.2. Gap Characteristics Across Frailty Phenotypes.

Gap number (A), gap percent (B) and gap duration (C) for daily EMG in nonfrail (dark gray bar), prefrail (black bar) and frail (light gray bar) females with Parkinson's disease. * Significantly different from nonfrail $(\mathrm{p}<0.02) . \%$, percent; s, seconds. Data is expressed as means \pm SEM. 
A.

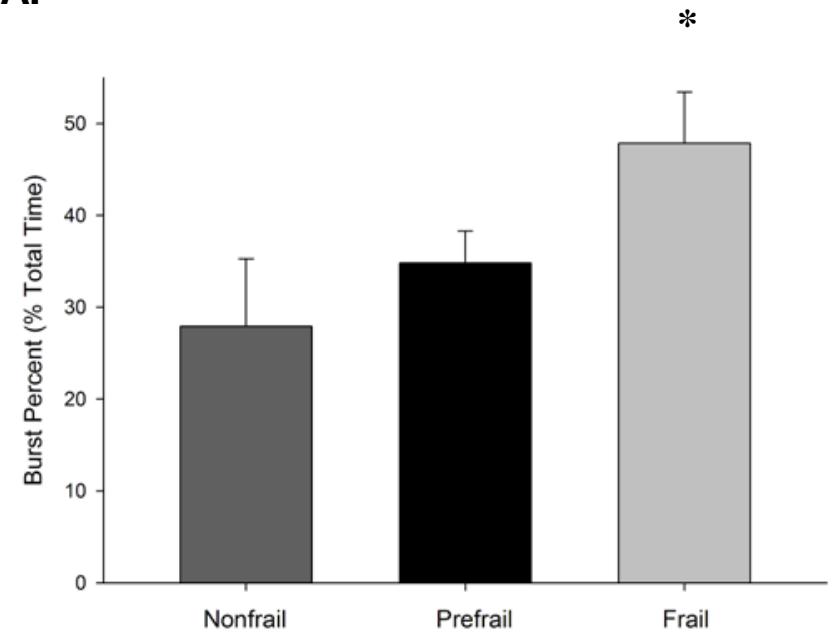

C.

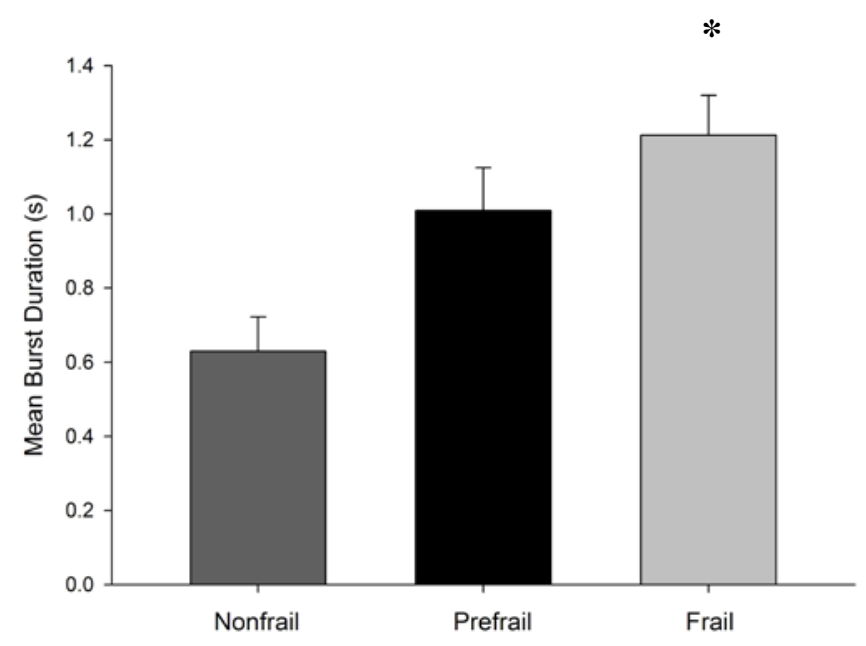

B.

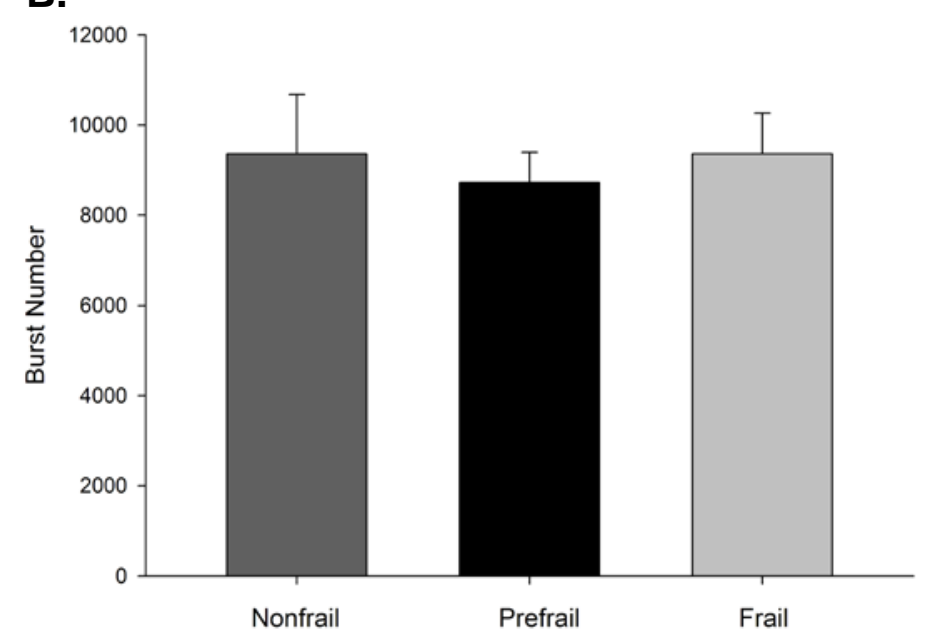

D.

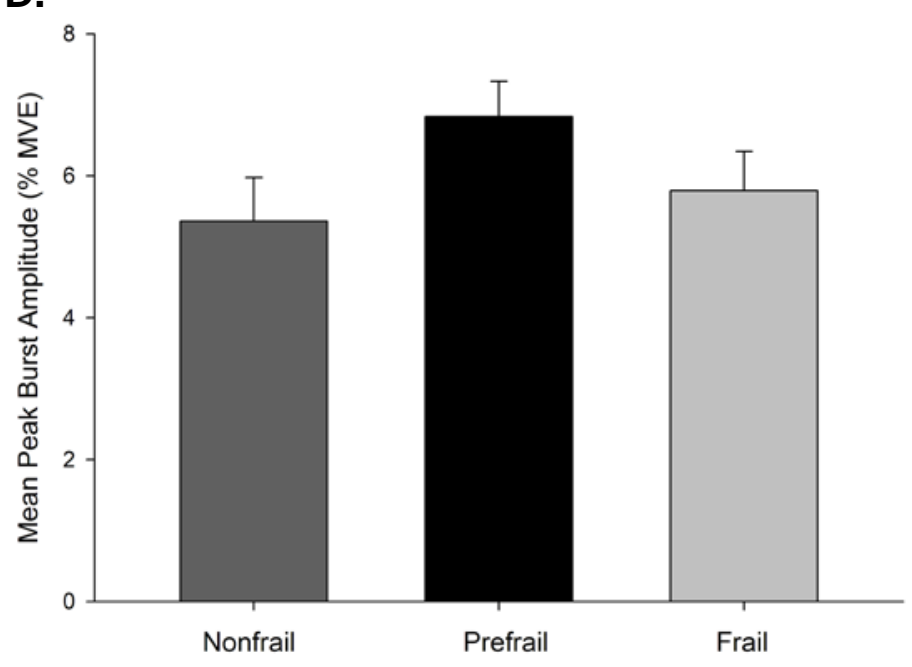

Figure 4.3. Burst Characteristics Across Frailty Phenotypes.

Burst percent (A), number (B), duration (C) and mean burst amplitude (D) for daily EMG in nonfrail (dark gray bar), prefrail (black bar) and frail (light gray bar) females with Parkinson's disease. * Significantly different from nonfrail $(\mathrm{p}<0.05)$. \%, percent; MVE, maximal voluntary exertion; $\mathrm{s}$, seconds. Data is expressed as means \pm SEM. 


\subsection{Discussion}

The current study measured daily muscle activity and quiescence in upper (BB, TB) and lower (VL, BF) limb muscles in females with PD. Females were categorized as nonfrail, prefrail and frail according to the CHSfi. The results of this investigation present difference in muscle activation characteristics (i.e., gaps, bursts) for frail compared with nonfrail females with PD. Frail females had decreased number of gaps and time occupied by gaps (gap percent) compared with nonfrail. Also, duration of bursts was greater in frail than nonfrail, contributing to greater overall period of time occupied by bursts (burst percent) in the EMG signal as frailty severity increased. This is the first study to examine the association between EMG recorded during routine daily activities and frailty in females with PD.

\subsubsection{Muscle Quiescence}

There is evidence of a greater number of gaps with each gap being of shorter duration in frail compared with nonfrail older females (Theou et al., 2010). However, in this investigation, frail females had fewer numbers of gaps and less time occupied by gaps compared with nonfrail. The decrease in EMG gaps observed in these frail females with PD can be interpreted as reduced muscle recovery time resulting from increased EMG activity (Harwood et al., 2008; Laursen et al., 2001). This increase in muscle activity, due to decreased periods of gaps might result in earlier onset fatigue (Blangsted et al., 2003; Laursen et al., 2001). The consequence of fatigue is decreased force generating capacity, which would require older adults to work at a higher threshold and lead to declines in functional performance. These functional declines may not be immediately apparent; however, continuous fatigue eventually accumulates and culminates in exhaustion (Allen, Lamb, \& Westerblad, 2008). Physical exhaustion is cited as one of the most disabling symptoms among persons with PD that inhibits physical function (Garber \& Friedman, 2003; Grace, Mendelsohn, \& Friedman, 2007) and contributes to physical frailty in persons with PD (Roland et al., 2012). Therefore, in frail females with PD reduced muscle quiescence likely 
causes a more continuous cycle of muscle activity, resulting in an earlier onset of muscle fatigue and eventually self-reported as exhaustion.

\subsubsection{Muscle Activity}

Theou and colleagues (2010) suggest muscle activity is less in frail compared to nonfrail females, since frail females report fewer bursts, although each burst is of longer duration than nonfrail and prefrail females. However, in this investigation no differences in burst number were observed between frailty phenotypes although burst duration was longer in frail compared to nonfrail females with PD. In PD, multiple bursting patterns are observed during the acceleration phase of movement and the number of bursts often increases with movement distance compared with healthy controls (Pfann et al., 2001; Robichaud et al., 2002). In this investigation EMG activation patterns attributed to PD progression would be similar across all females, since disease severity was similar between frailty phenotype groups. Longer burst durations may be indicative of slower rate of movement typical of older adults (Candow \& Chilbeck, 2005) and of the bradykinesia experienced by persons with PD. Greater burst duration presented in this investigation is consistent with previous evidence that indicates frail females exhibit more movement slowness (Theou et al., 2010). Longer duration of bursts suggests more muscle activity is required to initiate movement in frail women with $\mathrm{PD}$, thus it may take them longer to complete these actions. In addition, slowness of movement (i.e., gait speed) is one of the CHSfi criteria used to classify frailty phenotype (Fried et al., 2001). Bradykinetic movements may also suggest muscles are slow to relax, which is indicative of muscle fatigue. This increased fatigue is especially associated with physical tasks that use lower leg muscle where increased force is required, such as rising from a chair (Allen et al., 2008). 


\subsubsection{Muscle Differences}

Previous investigations reported greater burst activity in TB compared with BB, VL, and BF. Overall, muscle activity was greater in the upper limb muscles compared with lower limb during routine daily activities (Theou et al., 2010). On the contrary, decreased burst activity in TB compared with lower body muscle (BF) was seen in this investigation. The increased muscle activity in lower limb muscles relative to upper limb in persons with PD can be attributed to disease-related increased activation of leg flexors necessary for balance, gait and stance (Dietz, Ziistra, Prokop, \& Berger, 1995). This suggests increased activation of leg muscles in frail females with PD may be a result of disease-related declines in balance and gait stability.

\subsubsection{Physical Activity}

Physical activity can influence muscle strength and activation (Kent-Braun \& Ng, 1999; Enoka, 1997). Previous evidence reports muscle activity recorded during routine daily activities is highly correlated with physical activity in frail older females (Theou et al., 2010). In this investigation, physical activity was not different between frail and nonfrail females; however, the frail females had significantly fewer gaps and more bursts than the nonfrail females. This suggests the differences in muscle activation patterns observed in females with PD were not attributed to physical activity participation but rather to frailty severity. Despite the cited contributions of physical activity to frailty (Gill et al., 2002), previous evidence demonstrates that physical activity may not be the primary factor contributing to frailty in persons with PD as females with PD self-report greater leisure time activity participation (Roland, Cornett, Theou, Jakobi, \& Jones, In Press). This investigation determined that physical activity was not the primary contributor to changes in muscle activation across frailty phenotypes in persons with PD, rather the progression of frailty was the principal factor in altering muscle activity and quiescence. 


\subsubsection{Implications}

Thresholds of change in muscle activation patterns need to be identified to determine when a person becomes at-risk for greater frailty and functional dependence. Muscle activation patterns (gap, burst) discriminated frail from nonfrail females with PD. It should be noted that changes in muscle activation patterns are observed with various degrees of PD severity (Pfann et al., 2001). Also, decreases in muscle mass (“sarcopenia”) and strength attributed to the aging process can impact physical function, and contribute to differences in muscle activation patterns measured with EMG (Vandervoort, 2002). In this investigation, disease duration, disease severity (mild to moderate) and age were similar across all frailty phenotypes. Therefore, we can assume disease characteristics (i.e., severity, duration) and age had a limited contribution to differences in muscle activation patterns that distinguished frailty in these females with PD.

Changes in physiological capacity (muscle activation) may signal impending functional changes and resultant frailty. Early identification of physiological transitions toward frailty through EMG assessment may provide insight into the neuromuscular mechanisms that accelerate the progression of functional decline. Such a tool will enable the timely identification of subjects at risk (prefrailty) who can benefit from effective prehabilitation and rehabilitation programs. Rehabilitation studies demonstrate that exercise can have a positive effect on neuromuscular function and contribute to significant improvements in functional performance of persons with PD (David et al., 2012).

\subsection{Final Remarks}

Operational definitions of frailty vary and PD is commonly listed as an exclusion criterion for the analysis of frailty (Wong et al., 2010). Understanding how frailty relates to PD progression is important since females with PD are at greater risk of becoming prefrail-frail than males or persons without PD (Ahmed et al., 2008; Roland et al., 2012). The results of this 
investigation suggest that EMG can be used to dissociate frail females with PD from nonfrail based on muscle activation characteristics, specifically decreased number of gaps and greater burst duration during routine daily activities. Further investigation is required to provide insight into the identification of PD-specific mechanisms, specifically those that contribute to early stages of frailty progression (prefrailty). 


\section{Chapter 5: Physical Activity Across Frailty Phenotypes in Females with Parkinson's}

Disease $^{2}$

\subsection{Overview}

Frailty is a geriatric syndrome that results in an increased vulnerability to acute and chronic illness, falls and related injuries, and a general loss of functional independence (Bergman et al., 2004; Borges \& Menezes, 2011; Levers et al., 2006; Whiston et al., 2007). The Cardiovascular Health Study frailty index (CHSfi), proposes that frailty develops across a spectrum of phenotypes ranging from nonfrail to prefrail to frail (Fried et al., 2001). All stages of frailty are evident within community-dwelling populations, with over $70 \%$ of older adults expressing some frailty characteristics (Gill et al., 2006). Frailty is twice as prevalent in females as males and females typically display more frailty characteristics than males (Fried et al., 2001; Puts et al., 2005). Females experience increased frailty severity compared with males since they live longer and spend a greater proportion of life managing disability and disease (Leveille, et al., 2004; Mitnitski, Song, \& Rockwood, 2004; Puts et al., 2005).

Females with Parkinson's disease (PD) are at an increased risk of prefrailty (Roland et al., 2012), yet the presence of frailty in persons with PD may be misinterpreted as disease-related functional decline (Powell, 2008). Females with PD are typically older (Twelves et al., 2003), cite greater disability (Shulman, 2007) and experience more difficulty performing activities of daily living (ADL) than males with PD (Baba et al., 2005; Roland et al., 2011b). Recent evidence suggests that of the five CHSfi criteria, self-reported exhaustion best predicts frailty phenotype in females with PD (Roland et al., 2012). Exhaustion is a common complaint among older females, especially those with PD, and is strongly associated with inhibiting physical

\footnotetext{
${ }^{2}$ A version of this chapter is published. Roland, K.P., Cornett, K.M.D., Theou, O., Jakobi, J.M., \& Jones, G.R. (2012). Daily physical activity across frailty phenotypes in community-dwelling females with and without Parkinson’s disease. Journal of Aging Research, vol. 2012, Article ID 468156, 8 pages, 2012.
} 
activity and ADL participation (Garber \& Friedman, 2003; Grace et al., 2007; Nallegowda et al., 2004). The established relationship between reduced physical activity and frailty severity (Rothman, Leo-Summers, \& Gill, 2008) leads us to speculate that PD influences frailty through decreased participation in physical activity. Understanding how physical activity contributes to frailty in females with PD is important in directing management strategies aimed at maintaining functional independence. The objective of this study was to determine the contribution of daily physical activity on frailty phenotype in community-dwelling females with PD, compared to non-PD females.

\subsection{Methods}

Females greater than 50 years of age, living independently in the community, with mild to moderate PD severity (Hoehn \& Yahr stage I-III) were recruited through advertisements and support group presentations. Non-PD females were similarly recruited from the same local region. All subjects were able to ambulate independently (with or without gait-aids). Females with PD were in a steady clinical state (controlled by medication) and cognitively sound. Females with PD were instructed to continue daily dopamine medication as prescribed and no incidence of freezing of gait or severe dyskinesia episodes were reported during the testing day. All subjects provided written informed consent. The Clinical Research Ethics Board of the University of British Columbia granted ethical approval for this investigation.

Health history questionnaires, physical frailty criteria (CHSfi), self-reported physical activity, and set-up of daily physical activity monitors [accelerometer, global positioning systems (GPS)] were completed at the subject's home in the morning (8 10am). All PD subjects were assessed between one and two hours post anti-Parkinson's medication and controls were assessed one hour after breakfast. In older adults, reduction in daily physical activity, and associated physiological change, can be quantified using continuous objective physical activity 
monitors (i.e., accelerometers and global positioning systems, GPS) (Dijkstra, Kamsma, \& Zijlstra, 2010). In this study, a waist-born accelerometer and wrist-born GPS recorded physical activity and the subject wore these devices continuously for the entire testing day. The subject was then instructed to go about their typical daily activities, which they recorded in a written hourly log. The accelerometer, GPS, and physical activity logbook were collected approximately seven hours later (between 4-7pm) at the subject's home.

\subsubsection{Frailty Phenotype}

Frailty was categorized according to CHSfi (Fried et al., 2001) that includes five select criteria to determine a frailty phenotype (nonfrail, prefrail, frail). These criteria include: 1) Unintended weight loss (>10lbs in past 12 months); 2) Weakness (maximal handgrip-strength classified by body mass index, BMI); 3) Walk-speed (15ft at usual pace classified by height); 4) Self-reported energy expenditure (Minnesota Leisure Time Activities Questionnaire, MLTA); and 5) Self-reported exhaustion (Center for Epidemiological Studies depression scale, CES-D). Subjects were considered nonfrail if they satisfied none of the phenotypic criteria, prefrail if they satisfied one or two criteria, and frail if they satisfied three or more criteria (Fried et al., 2001).

\subsubsection{Accelerometer}

Daily physical activity was measured using an ActiTrainer accelerometer (Actigraph, LLC, Fort Walton Beach, FL) secured in a holster worn at the waist on the dominant side. The ActiTrainer ( $8.6 \times 3.3 \times 1.5 \mathrm{~cm}$; $51 \mathrm{~g})$ is a tri-axial solid-state accelerometer that was programmed to record 60-second epochs of data. Data was uploaded to ActiLife5 v.5.8.3 software (ActiGraph, LLC, Fort Walton Beach, FL). Physical activity intensity levels were categorized according to the cut-points described by Copeland and Eslinger (2009) for older adults. Sedentary activity was defined as 0 to 50 counts per minute, light physical activity as 51 to 1,040 counts per minute, and moderate-to-vigorous physical activity as greater than 1,041 counts per minute. Percentage of time spent at each level of activity was reported. Measurement 
outputs included total counts (i.e., daily step and activity counts, total minutes of activity) and intensity (i.e., percentage of time spent at sedentary, light, and moderate-vigorous activity intensity levels).

\subsubsection{Global Positioning System}

Global Positioning System (GPS) examines gross mobility outside an individual's home. GPS used in combination with the accelerometer can accurately assess physical activity within real-life environments (Webber \& Porter, 2009) and this can be applied to categorize stages of frailty (Karnik \& Mazzatti, 2009). Subjects wore a Garmin Forerunner 405 GPS watch (Garmin International Inc., Olathe, KS). GPS data were uploaded to the Garmin training center software (www.connect.garmin.com). Both the GPS and accelerometer units were synchronized to record minute-by-minute data. The GPS and accelerometer data were time-matched using the ActiGraph GPS Correlation Wizard v.1.0.0 (Actigraph, LLC, Fort Walton Beach, FL) and exported in a Microsoft Excel compatible format for analysis. Only physical activity completed outside the home, defined using Garmin Training Software and an accompanying Google Earth Map, was included in the analysis. Each subject’s hourly physical activity log was also compared to GPS outputs to confirm physical activity participation. Vehicle-generated activity was considered any recording that measured greater than $3 \mathrm{~m} / \mathrm{sec}$ for $>1 \mathrm{~min}$. All activity at speeds less than $3 \mathrm{~m} / \mathrm{sec}>1 \mathrm{~min}$ were included as subject physical activity. GPS measurement outputs included; total GPS distance (km, vehicle- and subject-generated) and total amount of physical activity time (min).

\subsubsection{Self-Reported Physical Activities}

Self perceived energy expenditure was assessed using the self-reported MLTA (Taylor, Jacobs, Schucker, Knudsen, Leon, \& Debacker, 1978), which was also used to determine energy expenditure as part of the CHSfi frailty assessment (Fried et al., 2001). Twenty activities were specified, including; walking for exercise, moderately strenuous household chores, mowing the 
lawn, raking the lawn, gardening, hiking, jogging, biking, exercise cycling, dancing, aerobics, bowling, golfing, calisthenics/general exercise, swimming, doubles tennis, singles tennis, and racquetball. Subjects who engaged in any physical activity during the previous two weeks recorded the number of sessions and their duration. Energy expenditure (kcal/week) was determined using metabolic equivalent (MET) score: (activity specific MET) x ((activity duration in minutes)/60) x ((number of sessions in past two weeks)/2). Total energy expenditure was calculated by summing expenditures over all activities.

\subsubsection{Statistical Analysis}

All analysis was performed using the Statistical Package for Social Science 18.0 (PASW Statistics 18.0, SPSS Inc. CIBM Somers, NY). Subject characteristics were compared between groups (non-PD, PD) and among frailty phenotypes (nonfrail, prefrail, frail) with a one-way ANOVA. The non-PD females were older than PD ( $p=0.005)$, nonfrail females were younger than prefrail and frail $(p=0.007)$ and prefrail were younger than frail $(p=0.03)$. Thus, age was used as a covariate in a two-way ANCOVA to assess differences in physical activity between disease states (PD; non-PD) and frailty phenotype (nonfrail; prefrail; frail). To evaluate the main effects a univariate analysis was performed for each dependent variable to identify contributions to the main effects of disease states and frailty phenotypes. Probability level was set at $\mathrm{p}<0.05$ and Tukey post hoc tests were used to probe statistical interactions. Spearman's rank correlation was used to evaluate physical activity measures relative to frailty phenotype in each group. The physical activity measures that were significantly correlated with frailty phenotype were entered into a multiple regression analysis model with frailty phenotype as the dependent variable and physical activity measures as the independent variables. 


\subsection{Results}

Fifteen persons with PD (65 \pm 9 years) and 15 non-PD controls (73 \pm 14 years)

participated. Both groups were categorized into frailty phenotypes according the CHSfi (Table 5.1.). In females with $\mathrm{PD}$, tremor in the upper limb was controlled with medication and subjects self-reported no freezing of gait or periods of dyskinesia over the course of the day. Further, any reports of rigidity and/or slowness of movement (bradykinesia) were mild and did not restrict routine daily activities. Comparison of physical activity between disease states and frailty phenotypes with age as a covariate determined nonfrail recorded less sedentary time, participated in more light-intensity physical activity and accumulated more steps compared with frail (Table 5.2.). Also, self-reported physical activity was lower in frail compared with the nonfrail and prefrail phenotypes (Table 5.2.). However, higher self-reported physical activity was reported in PD compared with non-PD, although the other physical activity variables did not differ between disease groups (Table 5.2.).

Table 5.1. Subject Characteristics Across Frailty Phenotypes

\begin{tabular}{|c|c|c|c|c|c|c|}
\hline & \multicolumn{3}{|c|}{$\underline{\mathrm{PD}}$} & \multicolumn{3}{|c|}{$\underline{\text { Non-PD }}$} \\
\hline & Nonfrail & Prefrail & Frail & Nonfrail & Prefrail & Frail \\
\hline Number & 4 & 7 & 4 & 8 & 4 & 3 \\
\hline Age & $69 \pm 1$ & $65 \pm 10$ & $63 \pm 11$ & $63 \pm 8^{\mathrm{ab}}$ & $79 \pm 14^{\mathrm{b}}$ & $90 \pm 6$ \\
\hline Body mass index $\left(\mathrm{kg} / \mathrm{m}^{2}\right)$ & $26.3 \pm 5.6$ & $22.9 \pm 4.3$ & $25.1 \pm 4.3$ & $23.2 \pm 5.7$ & $34.6 \pm 5.4$ & $32.5 \pm 12.2$ \\
\hline Number of medications & $4.0 \pm 2.0$ & $2.9 \pm 1.5$ & $3.3 \pm 2.6$ & $1.3 \pm 1.3$ & $6.0 \pm 4.2$ & $5.3 \pm 0.6$ \\
\hline mg CL / day & $366.7 \pm 57.7$ & $571.4 \pm 340.2$ & $825.0 \pm 330.4$ & & & \\
\hline CL only (N) & 2 & 3 & 2 & & & \\
\hline $\mathrm{CL}+\operatorname{pram}(\mathrm{N})$ & 2 & 2 & 0 & & & \\
\hline $\mathrm{CL}+$ enta $(\mathrm{N})$ & 0 & 0 & 1 & & & \\
\hline $\mathrm{CL}+$ enta + pram $(\mathrm{N})$ & 0 & 1 & 1 & & & \\
\hline $\mathrm{CL}+\operatorname{aman}+\operatorname{rop}(\mathrm{N})$ & 0 & 1 & 0 & & & \\
\hline Hoehn \& Yahr score & $1.8 \pm 0.8$ & $1.9 \pm 0.6$ & $2.5 \pm 0.4$ & & & \\
\hline
\end{tabular}

PD, Parkinson’s disease; kg, kilogram; m, meter; mg, milligram; CL, carbidopa-levodopa; pram, pramipexole; enta, entacapone; aman, amantadine; rop, ropinirole ${ }^{\text {a }}$ significantly different from prefrail ${ }^{\mathrm{b}}$ significantly different from frail. Reprinted with permission from the Journal of Aging Research. 
Table 5.2. Main Effects on Physical Activity Variables

\begin{tabular}{|c|c|c|c|c|c|}
\hline & PD & NonPD & Nonfrail & Prefrail & Frail \\
\hline Number & 15 & 15 & 12 & 11 & 7 \\
\hline Total steps & $3476 \pm 2814$ & $3731 \pm 3827$ & $5624 \pm 3309^{b}$ & $3019 \pm 3290$ & $1636 \pm 1599$ \\
\hline $\begin{array}{l}\% \text { time spent } \\
\text { sedentary }\end{array}$ & $61.7 \pm 14.1$ & $60.9 \pm 16.6$ & $49.4 \pm 9.5^{b}$ & $66.4 \pm 14.6$ & $71.9 \pm 11.3$ \\
\hline $\begin{array}{r}\% \text { time at } \\
\text { light activity }\end{array}$ & $32.2 \pm 10.6$ & $31.7 \pm 10.6$ & $39.4 \pm 7.4^{\mathrm{b}}$ & $28.6 \pm 10.2$ & $25.2 \pm 8.2$ \\
\hline MLTA & $3052.3 \pm 1611.6^{\mathrm{a}}$ & $2015.0 \pm 1517.4$ & $3045.0 \pm 1096.2^{b}$ & $2826.4 \pm 1790.4^{\mathrm{b}}$ & $1196.1 \pm 1493.7$ \\
\hline
\end{tabular}

Analysis adjusted for age; \%, percent; MLTA, Minnesota Leisure Time Activity; PD, Parkinson's disease; ${ }^{\text {a }}$ significantly different from non-PD, $\mathrm{p}<0.03{ }^{\mathrm{b}}{ }^{\mathrm{b}}$ significantly different from frail, $\mathrm{p}<0.05$. Reprinted with permission from the Journal of Aging Research.

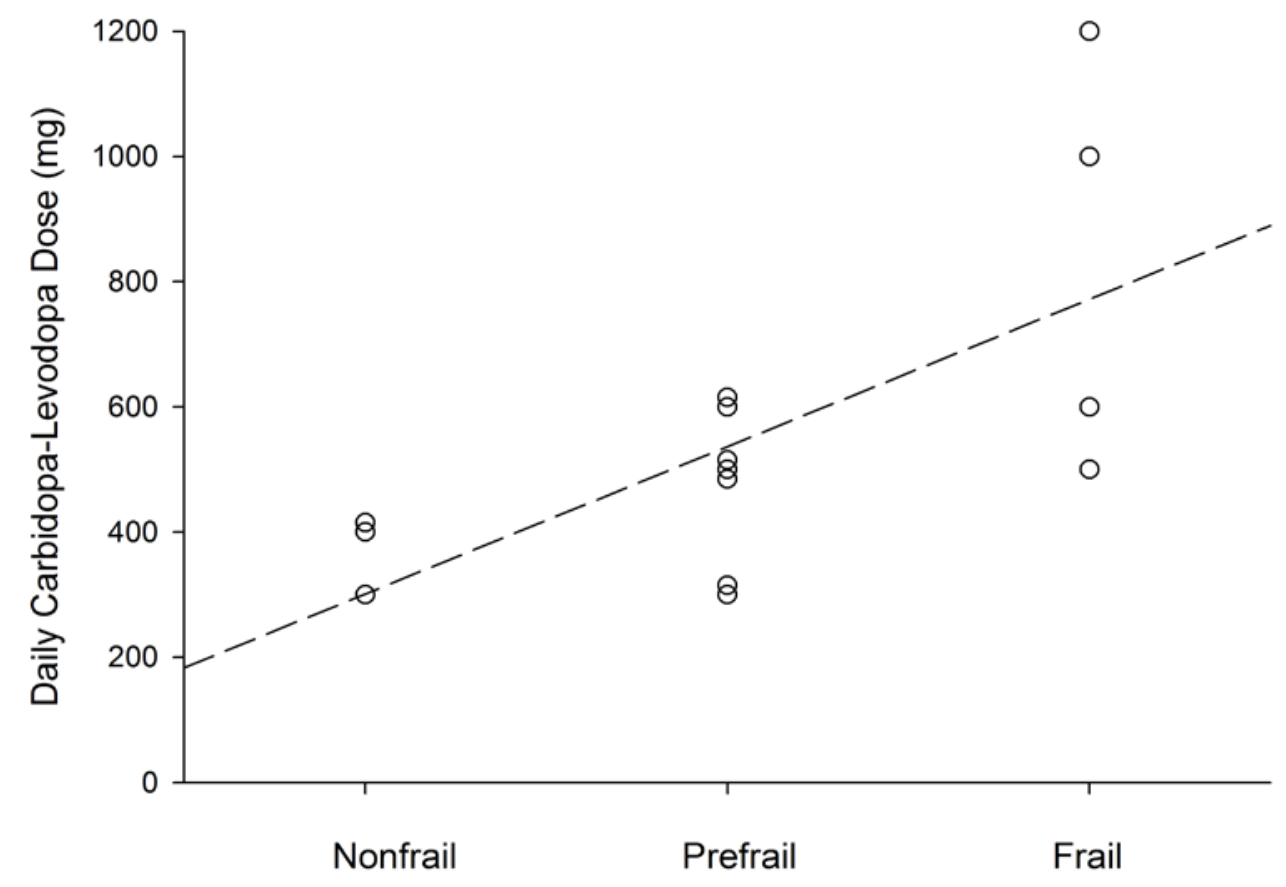

Figure 5.1. Relationship Between Frailty Phenotype and Carbidopa-Levodopa Dose

Increased step count is positively correlated with greater frailty severity $(r=0.61)$ in females with PD. Females with $\mathrm{PD}$ are represented by the open circles and dashed regression line. Reprinted with permission from the Journal of Aging Research. 
Table 5.3. The Association of Physical Activity Variables to Frailty in Control Females

\begin{tabular}{|c|c|c|c|c|c|}
\hline & \multirow{2}{*}{$\begin{array}{c}\text { ANOVA } \\
\text { Main effect } \\
\text { (p) }\end{array}$} & \multicolumn{2}{|c|}{ Spearman Correlation } & \multicolumn{2}{|c|}{ Linear Regression } \\
\hline & & $\begin{array}{c}\text { Correlation coefficient } \\
\text { (r) }\end{array}$ & $\begin{array}{l}\text { Significance } \\
\text { (p) }\end{array}$ & $\begin{array}{l}\text { Beta coefficient } \\
\qquad(\beta)\end{array}$ & $\begin{array}{l}\text { Significance } \\
\text { (p) }\end{array}$ \\
\hline \multicolumn{6}{|l|}{ Accelerometer- counts } \\
\hline Total steps counts* & 0.046 & -0.79 & .001 & 1.113 & 0.202 \\
\hline Total activity counts & NS & -0.75 & .001 & & \\
\hline Total activity time (min) & NS & -0.59 & .001 & & \\
\hline \multicolumn{6}{|l|}{ Accelerometer- intensity } \\
\hline $\begin{array}{r}\text { \% Time spent } \\
\text { sedentary* }\end{array}$ & 0.002 & 0.84 & .000 & 2.602 & 0.110 \\
\hline \% Time at light activity* & 0.012 & -0.82 & .000 & 1.225 & 0.226 \\
\hline \% Time at MV activity & NS & -0.81 & .000 & & \\
\hline \multicolumn{6}{|l|}{ GPS } \\
\hline $\begin{array}{l}\text { Total distance travelled } \\
\qquad(\mathrm{km})\end{array}$ & NS & -0.63 & .012 & & \\
\hline $\begin{array}{l}\text { Average travel speed } \\
\qquad(\mathrm{km} / \mathrm{h})\end{array}$ & NS & -0.58 & .023 & & \\
\hline $\begin{array}{r}\text { Physical activity time } \\
\text { (min) }\end{array}$ & NS & -0.54 & .036 & & \\
\hline \multicolumn{6}{|l|}{ Self-reported activity } \\
\hline MLTA (kcal/week)* & 0.007 & -0.82 & .000 & -0.386 & 0.108 \\
\hline
\end{tabular}

\%, percentage; GPS, global positioning system; h, hour; kcal, kilocalories; km, kilometers; MLTA, Minnesota Leisure Time Activity; min, minutes; MV, moderate-vigorous; NS, non-significant ( $>$ > 0.05); *significant main effects, therefore included in regression model. Reprinted with permission from the Journal of Aging Research. 

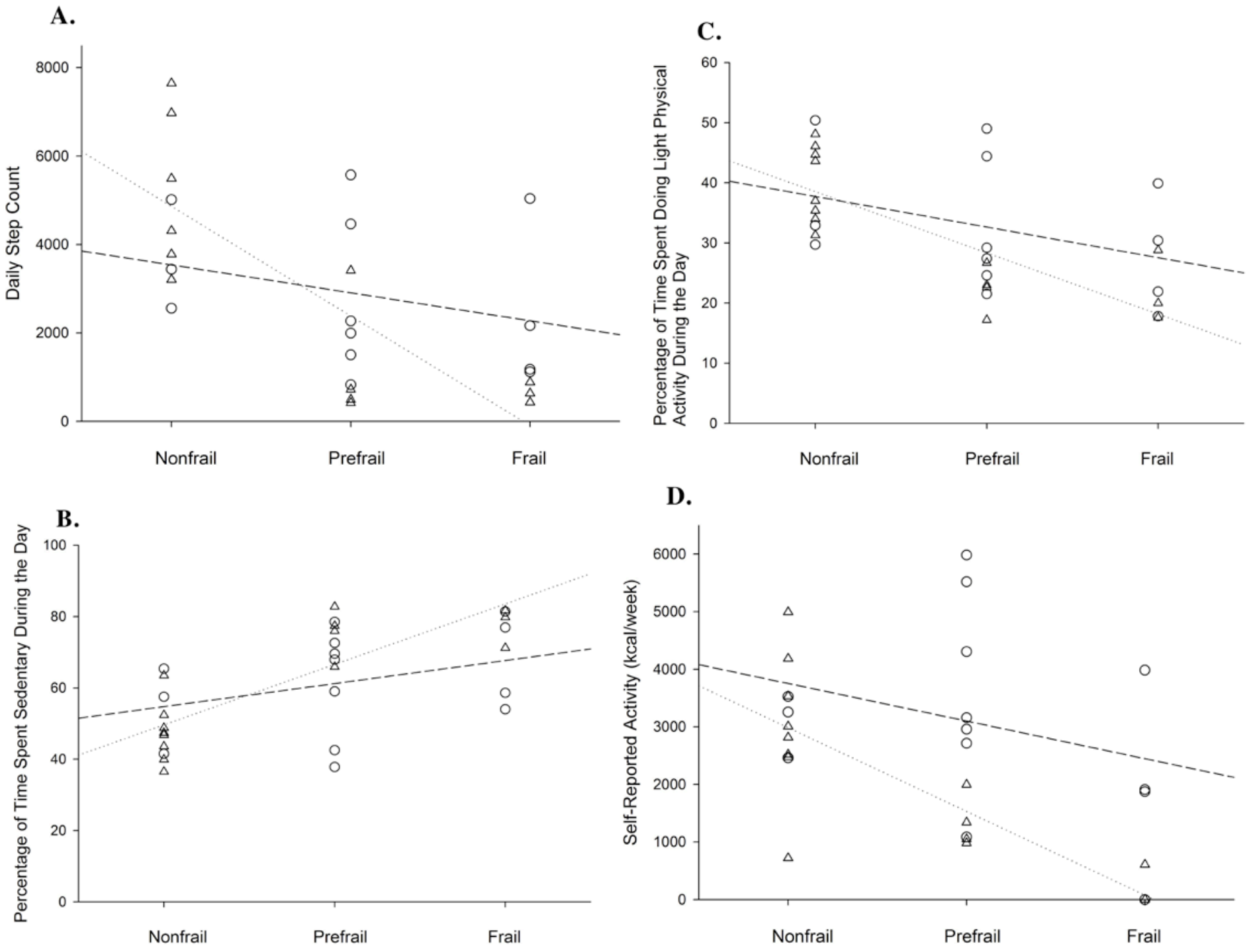

Figure 5.2. Relationship Between Frailty Severity and Daily Physical Activity

In females without PD, (A) total daily step count ( $\mathrm{r}=-0.79)$, (B) sedentary time $(\mathrm{r}=0.84),(\mathbf{C})$ light activity time $(\mathrm{r}=-0.82)$, and (D) self-reported activity ( $\mathrm{r}=-0.82)$ were correlated with frailty severity. Females with $\mathrm{PD}=$ open circles and dashed regression line; females without $\mathrm{PD}=$ open triangle and dotted regression line Reprinted with permission from the Journal of Aging Research. 


\subsubsection{Physical Activity and Frailty}

In PD, no physical activity variables were significantly related to frailty $(\mathrm{p}>0.29)$; however, daily dose of carbidopa-levodopa, including both controlled-release and active-release forms, was correlated with frailty $(r=0.61 ; \mathrm{p}=0.01)$ (Figure 5.1.). Other medication regimes for the management of PD and other comorbidities did not relate to frailty ( $p>0.42)$. Physical activity variables demonstrated a significant linear relationship with increasing frailty severity in non-PD females (Table 5.3.). Those physical activity variables that were significantly different between frailty phenotypes (Table 5.3.) were entered into individual regression models for nonPD. Low step counts, higher sedentary behaviour, reduced light activity and lower weekly selfreported energy expenditure accounted for $83.3 \%$ of the variance $(R=0.913 ; p=0.002$; Figure 5.2. A-D), but no single variable in this model best-predicted frailty ( $p=0.108$ to 0.226 ; Table 5.3.).

\subsection{Discussion}

This study examined the relationship between physical activity and frailty phenotype in community-dwelling females with PD and non-PD controls. Results of this study demonstrate physical activity (i.e., accelerometry counts and intensity, GPS and self-reported) was not related to frailty phenotype in this sample group of females with PD; however, lower daily physical activity was associated with increased frailty severity in non-PD females. Current literature demonstrates physical inactivity as an important determinant of frailty phenotype (Gill et al., 2002), and persons with PD have reduced levels of accelerometry-assessed physical activity compared to non-PD controls (Fujikane, Katayama, Hirata, \& Yokota, 1997). This study suggests daily physical activity participation may not be the primary factor associated with frailty phenotype in females with PD. It is unclear from our cross-sectional data if a causal relationship exists between physical activity and frailty. However, results presented here support 
future longitudinal investigation into how PD progression impacts physical activity and how these changes in symptom expression and physical activity influence physical frailty.

\subsubsection{Physical Activity and Frailty}

No relationship was demonstrated between daily physical activity and frailty phenotype in these females with PD. Although PD progression eventually debilitates motor performance, it is not likely the primary contributing factor to frailty in these females with PD. The results presented here do not necessarily exclude the contribution of physical activity to frailty, but rather suggest disease management may be a greater contributor to frailty. There is considerable variation in the manifestation of PD symptoms, which necessitates further large-scale investigation into PD-related contributors to frailty over the disease course. During the first 10 years of the disease, symptoms such as akinesia and festinating gait may not have progressed to the point at which they inhibit physical activity participation, which was the case with these subjects (Morris, 2000). In the initial stages of the disease, slowness to execute day-to-day activities (i.e., bradykinesia) is more common and this likely contributes to perceived exhaustion, which is a criterion for frailty. Thus, in community-dwelling persons with PD, self-reported exhaustion resulting from PD symptoms contributes to frailty (Roland et al., 2012), rather than reduced daily physical activity.

Results of this study indicate that although physical activity was not related to frailty in PD, females with PD self-reported greater leisure time activities compared to non-PD controls, regardless of frailty severity. This finding argues against previous research that states community-dwelling persons with PD have significantly less daily physical activity energy expenditure (measured using MLTA questionnaire) compared with controls, although the study sample included only males (Toth, Fishman, \& Poehlman, 1997). We speculate that increased self-reported leisure activity in these females with PD is due to the benefits of physical exercise being well recognized in the PD population to improve motor performance, functional and 
cognitive ability, safety and confidence in ADL (Morris, 2000). Persons living with PD are constantly encouraged to remain active despite disease-associated barriers (i.e., exhaustion) (Shaw, Vancouver Coastal Health). Previous research has demonstrated older adults with an increased risk of mortality, like persons with PD, adhere better to exercise programs compared to general community-dwelling older adults (Morey, Pieper, Crowley, Sullivan, \& Puglisi, 2002). Therefore, we speculate that persons with PD may be involved in more physical activity and better adhere to exercise recommendations compared with community-dwelling counterparts because of PD-associated disease symptoms, regardless of frailty phenotype.

Unlike females with PD, physical activity influences frailty severity in communitydwelling females without PD. The contribution of physical activity to frailty in females without PD may be attributed to reduced mobility, less activity intensity and restricted life-space (i.e., the spatial area a person moves through during daily life) (Fallah, Mitnitski, Searle, Gahbauer, Gill, \& Rockwood, 2011; Baker, Bodner, \& Allman, 2003). These physical and environmental challenges contribute to further reductions in physical activity and exacerbate declining functional reserves (Xue et al., 2008), encouraging transition to greater frailty severity. Remaining active during old age is important as sedentary lifestyle significantly increases risk of developing multiple chronic diseases and premature mortality (Xue et al., 2008). Disease prevalence and functional impairment increases with age (Rockwood, Fox, Stolee, Robertson, Beattie, 1994), which places the older adult at greater risk of frailty (Fried et al., 2001). However, it is unclear whether age and age-related characteristics are associated with frailty in females with PD. This sample was randomly recruited from a population of females living independently in their own homes. Persons with PD seek institutional care sooner than non-PD counterparts (Aarsland, Larsen, Tandberg, \& Laake, 2000), suggesting they may reach frailty sooner. Future longitudinal research may investigate onset of frailty in females with PD and follow frailty 
progression throughout the disease course. Also, further information on the contribution of physical activity to frailty onset and progression in PD is needed.

\subsubsection{Parkinson's Disease Management and Frailty}

Accelerometers have been used in several research fields to monitor daily physical activity; monitoring of steps using waist-born accelerometers is feasible in PD populations and provides useful feedback on freezing (i.e., sudden inability to move, especially in the legs during walking), as well as long-term daily activity. Results of this study indicate that daily physical activity does not contribute to frailty phenotype in community-dwelling females with PD; however, daily carbidopa-levodopa dose was significantly related to frailty. Medication regimes for subjects in this study included medications for the management of PD (i.e., carbidopalevodopa, entacapone, pramipexole, amantadine, ropinerole) and other conditions, such as anxiety/depression, blood pressure, migraine, muscle pain and inflammation, post-menopause symptoms, and difficulty sleeping. Multiple medication use in PD is associated with functional decline and high fall-risk (Ashburn et al., 2001); both of which are indicative of frailty. In addition, dopamine deficiency in PD may result in physical exhaustion (Lou, Kearns, Bnice, Oken, Sexton, \& Nutt, 2003), increasing functional dependence and risk for frailty. Therefore, the impact of PD medication regime on physical function and frailty requires further inquiry.

In addition to motor impairments, persons with PD face secondary symptoms that impact basic daily function, such as depression, cognitive impairments and non-motor symptoms that increase functional dependence through increased anxiety, social isolation and confusion (Rahman et al., 2008). Cognition is considered an important component of frailty (Avila-Funes et al., 2009) and is also associated with adverse PD outcomes (Rahman et al., 2008). Cognitive function can be influenced by depression and physical decline, and females with PD report greater incidence of both compared with males (Baba et al., 2005). Also, females with PD report greater symptom-related stress and sleep disturbances than males (Scott, Borgman, Engler, 
Johnels, \& Aquilonius, 2000; Smith, Ellgring, \& Oertel, 1995). The importance of these nonmotor symptoms cannot be underestimated, however investigation of their contribution to frailty in PD are beyond the scope of this study. Future research should consider how PD symptoms (motor and non-motor), disease severity, duration, other comorbidities, polypharmacy, and age impact frailty severity in both males and females with PD.

\subsubsection{Implications}

\subsubsection{Independent-Living Populations and Neurological Disorders}

The progressive nature of PD and related symptoms such as bradykinesia and tremor causes persons with PD to seek long-term care earlier than the general older adult population (Aarsland et al., 2000). Females with PD may be more vulnerable to frailty than persons without neurological disorder because of PD-related systems that exacerbate the frailty phenotype. Therefore, it is important to make an early identification of frailty in community-dwelling persons, especially those with neurological disorders who express a frailty phenotype earlier than the general population.

\subsubsection{Frailty Management}

Physical inactivity is directly linked to declining physiological reserve capacity, defined as adaptive responses (i.e., heart rate, blood pressure, respiration) that enable us to perform tasks or overcome external stresses (Lipsitz, 2004). In persons with PD the complexity of symptom presentation results in a reduced adaptive capacity. These losses of adaptive capacity, related to aging or disease, lead to declining functional independence and are a determinant of frailty (Gill et al., 2002). Co-morbidities and clinical symptoms interact between frailty and PD making identification of frailty in persons with PD challenging to diagnose (Powell, 2008). Few studies have examined how to accurately identify frailty in this population (Ahmed et al., 2008; Lauretani et al., 2012). Early identification of frailty in persons with PD is relevant since over 
half of older adults living independently in their own homes are at-risk for frailty and subsequent functional decline (Gill et al., 2006). Due to the transitional nature of both frailty and PD, the majority of disease progression occurs long before the individual requires institutional care (Murtch et al., 1986). As PD severity increases, symptoms become aggravated, further exacerbating underlying frailty (Lauretani et al., 2012). Awareness of frailty during the initial stages of PD development may contribute to improved management strategies that delay and/or reverse frailty factors and preserve functional independence (Gill et al., 2006). This study demonstrated that frailty was related to decreased physical activity in community-dwelling nonPD, and symptom management in PD females. Knowledge gained from this study can be used to inform effective strategies for identifying prefrailty in non-PD females. This information can be applied to the development and delivery of timely support that addresses age- and diseaseassociated declines in function. Preventing functional decline has important implications for healthcare resource use in PD and non-PD older adults, as well as reducing physical, emotional, social and financial problems attributed to frailty (Gill et al., 2002).

Currently, there is little evidence on how PD specifically impacts females and contributes to increased risk of prefrailty. Greater risk of frailty may be a consequence of greater functional declines (Roland et al., 2012), distress and cognitive impairment (Avila-Funes et al., 2009), all resulting in functional dependence (Leveille et al., 2004). In females with PD, physical activity participation should be aimed at managing PD symptoms. Addressing PD symptoms, through physical activity and medication, will incidentally contribute to frailty management. Emphasis, therefore, should be placed on managing frailty within the context of PD symptoms (Powell, 2008). Further collaboration is crucial between neurological, geriatric practice, and physical therapy/rehabilitation in terms of frailty assessment, progression, and addressing complications resulting in declining physical activity that may culminate in frailty. 


\subsection{Final Remarks}

Physical activity influences frailty expression in older females without PD, surprisingly no relationship between physical activity and frailty was found in our sample of females with PD. In PD, disease management may better predict frailty severity. Further study is warranted to establish how PD-associated characteristics (i.e., polypharmacy) contribute to frailty and how physical activity participation interplays with the complex progress of frailty within PD. This study suggests PD-associated symptoms motivate community-dwelling females with PD to engage in leisure-time physical activities. Considering its vast impact in the community and on healthcare resources, identification of early frailty and management of resulting disability remains a priority area for geriatric research. Ultimately, enabling older adults to remain physically active promotes independence in daily living and empowers positive aging, although it may not protect females with PD from becoming frail. 


\section{Chapter 6: Quality of Life as a Determinant of Frailty Phenotype in Persons with}

Parkinson's Disease ${ }^{3}$

\subsection{Overview}

Frailty and Parkinson’s disease (PD) have discrete clinical signs and underlying pathologies, yet frailty often coexists with PD in older adults (Ahmed et al., 2008; Lauretani et al., 2010; Powell, 2008). PD primarily presents as a loss of motor ability associated with postural instability and gait impairment. The effect of PD symptoms on quality of life (QoL) is increasingly recognized as an important component of care (Behari, Srivastava, \& Pandey, 2005; Powell, 2008). Poor QoL is associated with frailty in older adults (Fried et al., 2001), yet little is known about how QoL contributes to frailty in persons with PD.

The presence of interacting symptoms in community-dwelling individuals with PD can contribute to functional dependence in daily life activities, resulting in frailty (Lauretani et al., 2010). It is challenging for healthcare professionals to recognize frailty in persons with PD (Gill et al., 2006; Powell, 2008), but early identification would enhance QoL (Behari et al., 2005). Thus, recognition of early frailty in persons with PD is warranted. This study aims to determine which physical frailty and QoL characteristics best discriminate between frailty phenotype in community-dwelling persons with PD.

\subsection{Methods}

Persons diagnosed with PD (Hoehn \& Yahr stages I-III) who were able to ambulate independently and were cognitively intact and clinically stable were recruited from the community and assessed in the morning one to two hours after they took their medication. The

${ }^{3}$ A version of this chapter is published. Roland, K.P., Jakobi, J.M., Powell, C., \& Jones, G.R. (2012). Quality of life as a determinant of frailty phenotype in community-dwelling persons with Parkinson's disease. Journal of the American Geriatrics Society, 60(3), 590-592. 
Cardiovascular Health Study frailty index (CHSfi) captures physical characteristics associated with early frailty development and uses five criteria to develop nonfrail, prefrail, and frail phenotypes (Fried et al., 2001). QoL was assessed using the Parkinson’s disease Questionnaire39 (PDQ-39), a disease-specific self-assessment tool (Jenkinson, Fitzpatrick, Peto, Greenhall, \& Hyman, 1997). All subjects provided written informed consent. The clinical research ethics board at the University of British Columbia granted ethical approval for this investigation.

Bivariate analysis was used to measure the strength of the association between CHSfi frailty score, QoL, and PD characteristics. Logistic regression and proportional odds ratio (OR) models were used to assess the relationship between frailty criteria, QoL, and PD characteristics. A 95\% confidence interval (CI) and .05 significance value were used.

\subsection{Results}

The sample consisted of 29 (aged $66.4 \pm 8.5,12$ female) community-dwelling persons with PD (duration $7.2 \pm 4.6$ years; Hoehn \& Yahr score $2.0 \pm 7$ ). CHSfi criteria classified nine as nonfrail (1 female), 19 as prefrail (11 female), and one as frail (0 female) (Fried et al., 2001). Nonfrail persons with PD had shorter disease duration $(p=.04)$, less disease severity $(p=.001)$ and better QoL $(\mathrm{p}=.02)$ than prefrail persons. PD severity (Figure 6.1.A) and worse QoL (Figure 6.1.B) were strong predictors of frailty phenotype. Other predictors of frailty were selfreported exhaustion according to the CHSfi (coefficient of determination $\left(\mathrm{R}^{2}\right)=0.44$ ) and mobility scored from the PDQ-39 $\left(\mathrm{R}^{2}=0.32\right)$. Being female was significantly associated with frailty status $(\mathrm{OR}=9.78,95 \% \mathrm{CI}=1.0-93.5)$. 

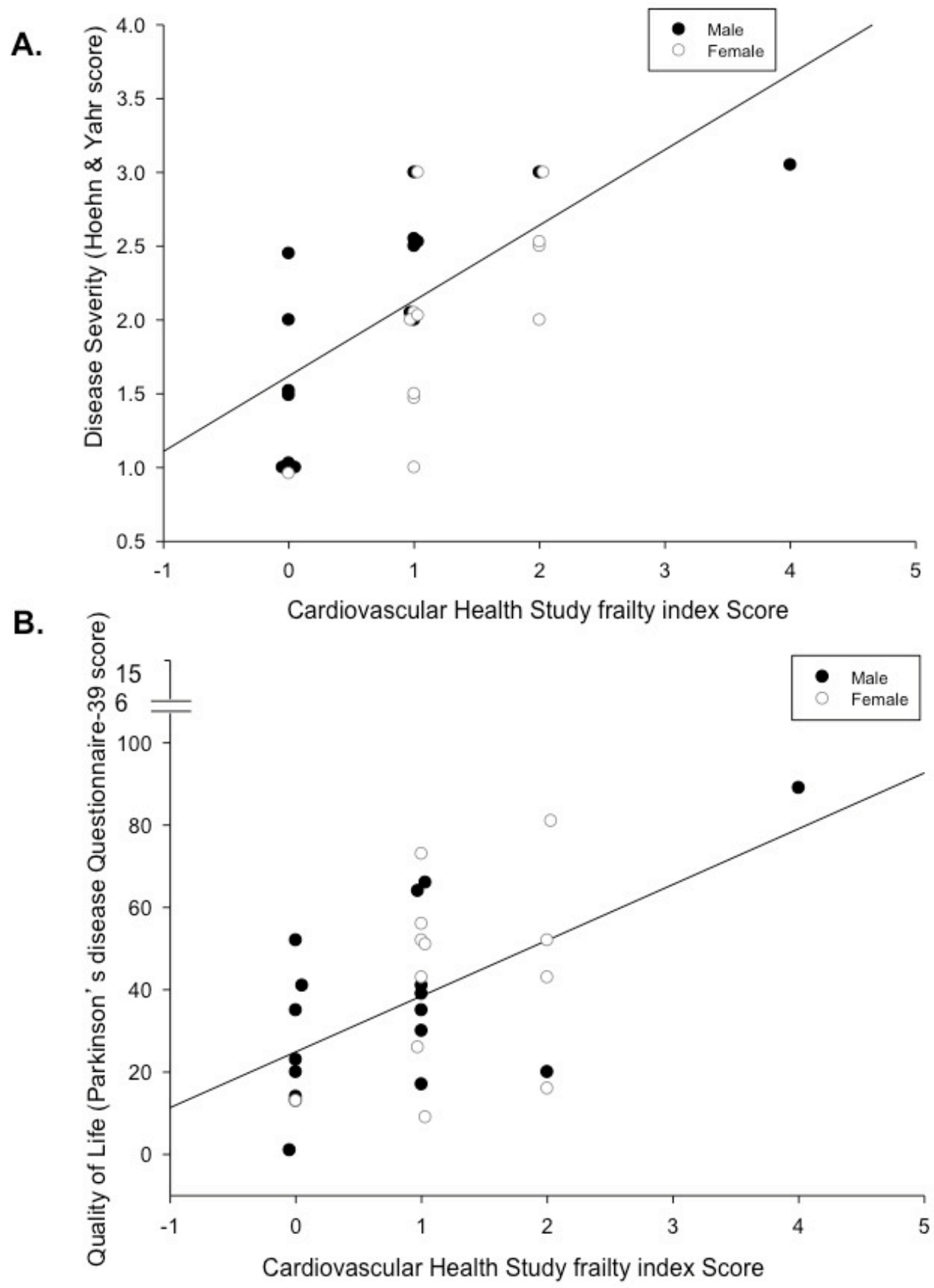

Figure 6.1. Relationship Between Frailty, Quality of Life and Parkinson's Disease

(A) Relationship between frailty score and disease severity. Higher frailty score is positively correlated with greater disease severity (correlation coefficient $(r)=0.65$ ). Disease severity predicted frailty status, and this model accounted for $43 \%$ of the variance (coefficient of variation $\left(R^{2}\right)=0.43$ ). (B) Relationship between frailty score and quality of life (QoL). Higher frailty score is positively correlated with poorer QoL (higher PDQ-39 score) $(r=0.53)$. QoL predicted frailty status, and this model accounted for $12 \%$ of the variance $\left(R^{2}=0.12\right)$. Males with PD $=$ closed circles; females with PD = open circles. Reprinted with permission from the Journal of the American Geriatrics Society. 


\subsection{Discussion}

This study demonstrates that all frailty phenotypes are measurable in persons with PD; greater disease severity, worse QoL, and self-reported exhaustion criteria contribute to frailty status; and that females with PD are more vulnerable to prefrailty than males. It is important to identify these factors early, because frailty often remains unrecognized during the initial stages of PD (Lauretani et al., 2012). Earlier identification may delay or reverse frailty-associated dependences (Gill et al., 2006).

Exhaustion is a disabling feature of PD and a common complaint in older adults. It may result from changes within the peripheral or central nervous system, or psychological factors (Grace et al., 2007), in addition to declining physiological capacity and associated weakness. Therefore, exhaustion as a consequence of PD can lead to functional decline and isolation and result in greater vulnerability to frailty.

This study is novel because it highlights the link between QoL and frailty in persons with PD, specifically its influence on mobility-related QoL. PD-associated motor complications may also make persons with PD anxious about how they are perceived (Chapuis, Ouchane, Metz, Gerbaud, \& Durif, 2011), and both may increase the likelihood of withdrawal and functional dependence. Further insight into mobility-related QoL in persons with PD is needed to improve early recognition of functional dependence and how this may attenuate frailty in PD. Future investigation should explore the contribution of social well-being to QoL in PD and frailty as independent conditions.

These results suggest that community-dwelling females are more vulnerable to prefrailty than males. Greater frailty in females with PD may be due to greater disability and functional dependence and poorer QoL than in males (Behari et al., 2005; Roland et al., 2011b). Knowledge gained from this study informs future recognition of sex-specific risk factors of frailty in PD. 


\subsection{Final Remarks}

This study provides new insight into factors that identify frailty phenotypes in community-dwelling persons with PD and suggests a relationship between QoL and frailty in persons with PD. Frailty identification in PD should be a priority issue for prehabilitative geriatric services (Lauretani et al., 2012), especially with decreasing availability of neurologist care. Understanding complex interactions between frailty and PD will enable earlier identification of frailty. Knowledge gained can be used to inform management strategies that effectively target concomitant symptoms, preserving functional independence and improving QoL in persons with PD. 


\section{Chapter 7: Concurrence of Frailty and Parkinson's Disease ${ }^{4}$}

\subsection{Overview}

Parkinson's disease (PD) is a progressive neurological disorder where non-motor, neuropsychiatric and motor impairments significantly impact function during daily life. Functional ability is defined as the capacity to perform purposeful movement and tasks necessary for independent daily living (Avlund et al., 1996). Since no cure is available, different treatment options are used to manage PD symptoms. Symptom relief is commonly obtained using dopaminergic medication such as carbidopa-levodopa therapy (Jankovic, 2008). Medication regimes for PD often include multiple pharmaceutical agents (i.e., anticholinergics, amantadine, dopamine agonists) that improve carbidopa-levodopa effectiveness (Lang \& Lees, 2002). Each patient's treatment regime is prescribed and adjusted to meet their specific needs. Indeed, dopaminergic medication is the gold standard for symptom management and enables persons with PD to maintain some level of function and maximize quality of life (QoL) (Fitzpatrick et al., 1997).

Functional decline is influenced by frailty severity, a geriatric syndrome that increases vulnerability to adverse health outcomes (i.e., disability, hospitalization, mortality). The Cardiovascular Health Study frailty index (CHSfi) measures select criteria that classify individuals according to their physical ability and identifies those at-risk for frailty (i.e., prefrailty) (Fried et al., 2001). Frequently, PD is listed as an exclusion criterion for the analysis of frailty (Wong et al., 2010); however, females with PD are at increased risk for prefrailty (Roland et al., 2012). Physical frailty and PD characteristics often overlap, which make identification of frailty in persons with PD challenging. This suggests PD-specific attributes that

${ }^{4}$ A version of this chapter is published. Roland, K.P., Cornett, K.M.D., Theou, O., Jakobi, J.M., \& Jones, G.R. (2012). Concurrence of frailty and Parkinson's disease. The Journal of Frailty and Aging, 1(3), 123-127. 
relate to frailty need to be determined. Understanding how frailty relates to PD progression is important in females because they are more likely to be frail and suffer greater functional decline than males or persons without PD (Fried et al., 2001; Puts et al., 2005; Roland et al., 2011b; Roland et al., 2012).

Disease prevalence and functional impairment increases with age (Rockwood et al., 1994), which places the older adult at greater risk for frailty (Fried et al., 2001). It is unclear whether these are related to frailty in persons living with PD. The likelihood of frailty is greater in older adults when two or more co-morbidities are present (Fried et al., 2001; Fried, Ferrucci, Darer, Williamson \& Anderson, 2004). Research suggests females suffer from more chronic, debilitating diseases compared with males and spend significantly more time managing comorbidity-related disability. Multiple medication use is most evident in older adults with multiple comorbidities. Polypharmacy increases the likelihood for interactions and/or adverse drug reactions (Hilmer \& Gnjidic, 2009). Comorbidity and polypharmacy may increase risk for frailty in older adults through physiological changes, inactivity and adverse reactions (Hilmer \& Gnjidic, 2009; Edelberg, Shallenberger, Hausdorff, \& Wei, 2000; Fried et al., 2004). In persons with PD, management strategies often consist of multiple medication prescriptions, with constant monitoring of dose adjustments to reflect symptom progression (Lang \& Lees, 2002); however, no study has yet determined the association of polypharmacy and other disease-related characteristics (disease severity, disease duration) on frailty in females with PD.

The purpose of this investigation is to determine age- and disease-related characteristics that best explain frailty in community-dwelling females with PD. Recognizing early frailty is important because it can improve patient care and direct treatment for both concurrent conditions in persons with PD (Lauretani et al., 2012; Powell, 2008). It is hypothesized that increasing age, number of comorbidities, pharmaceutical use, disease severity and duration will relate to frailty 
in community-dwelling females with PD. Identification of frailty in PD is challenging, given that those with PD have not traditionally been included in frailty investigations.

\subsection{Methods}

\subsubsection{Study Design}

Females, greater than 50 years of age, living in community settings, with mild to moderate PD (Hoehn \& Yahr stage I-III), were recruited to participate in this study through advertisements and support group presentations. Similarly, non-PD females were sampled from the same local community using similar recruitment strategies. All subjects were able to ambulate independently (with or without gait-aids). Subjects with PD were in a steady clinical state (controlled by medication) and found to be cognitively intact. Subjects' self-reported age and disease-related characteristics were collected using a health history questionnaire. The health history questionnaire assessed subject's health status and age, height, body weight, smoking history, surgical history, presence of comorbidities, allergies, visual or auditory difficulties, use of a gait-aid and current medication regime. All subjects completed the health history questionnaire and physical frailty assessments at their home in the morning (8 10am). Subjects with PD were asked to complete the in-home assessment one to two hours after taking their regular anti-Parkinson's medication, while healthy controls were assessed one hour after breakfast. The Clinical Research Ethics Board of the University of British Columbia granted ethical approval for this investigation.

\subsubsection{Frailty Phenotype}

Frailty severity was classified in females with and without PD according to the CHSfi. The CHSfi uses five physical frailty criteria to classify people according to their physical functional ability. As measured by Fried and colleagues (2001), frailty was operationalized according to the following characteristics: weight loss ("Have you lost more than $10 \mathrm{lbs}$ unintentionally in the past year?”), exhaustion (“How often in the last week did you feel that 
everything you did was an effort?” and “How often in the last week did you feel that you could not get going?”), weakness (maximal dominant hand-grip strength, kg), slow gait-speed (15ft walk-time at steady pace, sec), and energy expenditure (Minnesota Leisure Time Activity Questionnaire, kcals/week). Frailty severity increases with the accumulation of more frailty criteria (i.e., two to three criteria = prefrail, three to five criteria $=$ frail).

\subsubsection{Statistical Analysis}

All analysis was performed using the Statistical Package for Social Science 18.0 (PASW Statistics 18.0, SPSS Inc. (CIBM Somers, NY). Subjects’ age and disease-related characteristics were compared across frailty phenotypes using analysis of variance (ANOVA). Spearman’s rank correlation coefficients expressed the separate relationships between frailty classified by CHSfi (ordinal variable) and subject characteristics (continuous variables) in PD and non-PD females. In addition, CHSfi criteria were correlated to disease severity using Pearson's product-moment correlation. Any characteristics that significantly correlated to frailty were entered as variables in the regression model. Regression analysis was used to determine variables that best explain frailty, with frailty severity as the dependent variable and age and disease-related characteristics that were significantly correlated to frailty as the independent variables. Predictors of frailty were determined individually for PD and non-PD females using this regression model. These models established the individual variables that best predicted frailty in each sample. A significance of $\mathrm{p}<0.05$ was used. 


\subsection{Results}

The study sample included 17 PD (mean age $=66 \pm 8.5$ years) and 18 non-PD females (mean age $=72 \pm 13.2$ years $)(p=0.10)$. Results indicated that subjects with PD had significantly less comorbidity than non-PD females $(p=0.045)$ and were of moderate disease severity $(\mathrm{H} \& \mathrm{Y}=2.06 \pm 0.68)$ with average disease duration of $7 \pm 6.6$ years (Table 7.1.). Relationships between age and disease-related characteristics and total CHSfi score are reported in Table 7.2. Severity of PD was correlated with meeting the CHSfi criteria for self-reported exhaustion ( $r=0.47, \mathrm{p}=0.004)$; no other individual CHSfi criterion was related to PD severity or duration.

Neither PD severity $(p=0.08)$ nor disease duration $(p=0.23)$ was correlated with CHSfi frailty phenotype. However, daily carbidopa-levodopa dose was related to frailty phenotype $(\mathrm{r}=$ $0.61 ; \mathrm{p}=0.01$ ). The regression model with carbidopa-levodopa dose explained $24.5 \%$ of the variance in frailty phenotype in $\mathrm{PD}$ females $(\mathrm{R}=0.50 ; \beta=0.50 ; \mathrm{p}=0.015$; observed power $=$ 0.63) (Table 7.2., Figure 7.1.A.). Subject characteristics in non-PD females that related to greater frailty severity in this sample included; increased age $(r=0.73)$, polypharmacy $(r=0.67)$, and greater number of comorbidities $(r=0.50)(p=0.001$ to 0.033$)$. These significant variables were entered into the regression model and explained $76.7 \%$ of the variance in frailty phenotype (observed power $=0.9$; Table 7.2.). Individually, age and number of comorbidities bestexplained frailty within the regression model (Table 7.2., Figure 7.1.B,C.). 
Table 7.1. Subject Characteristics Across Frailty Phenotypes

\begin{tabular}{|c|c|c|c|}
\hline & $\begin{array}{l}\text { Nonfrail } \\
\text { (0 criteria) }\end{array}$ & $\begin{array}{c}\text { Prefrail } \\
\text { (1-2 criteria) }\end{array}$ & $\begin{array}{c}\text { Frail } \\
\text { (3-5 criteria) }\end{array}$ \\
\hline \multicolumn{4}{|l|}{ Non-PD Females: } \\
\hline Number & 10 & 5 & 3 \\
\hline Age (years) & $65 \pm 7.5$ & $75 \pm 14.0$ & $90 \pm 5.6$ \\
\hline BMI $\left(\mathrm{kg} / \mathrm{m}^{2}\right)$ & $23.25 \pm 5.2$ & $31.40 \pm 8.7$ & $24.29 \pm 5.1$ \\
\hline Number of medications & $1.00 \pm 1.2$ & $6.00 \pm 3.5$ & $5.33 \pm 0.6$ \\
\hline Number of comorbidities & $1.20 \pm 1.2$ & $4.60 \pm 2.9$ & $5.33 \pm 1.2$ \\
\hline \multicolumn{4}{|l|}{ PD Females: } \\
\hline Number & 4 & 8 & 5 \\
\hline Age (years) & $68 \pm 2.6$ & $65 \pm 9.4$ & $65 \pm 11.2$ \\
\hline BMI $\left(\mathrm{kg} / \mathrm{m}^{2}\right)$ & $25.35 \pm 5.2$ & $23.0 \pm 4.0$ & $23.92 \pm 4.3$ \\
\hline Number of medications & $4.25 \pm 1.7$ & $3.25 \pm 1.8$ & $3.00 \pm 2.3$ \\
\hline Number of comorbidities & $2.00 \pm 1.2$ & $0.88 \pm 1.1$ & $1.20 \pm 1.1$ \\
\hline Disease duration (years) & $2.8 \pm 2.2$ & $11.1 \pm 7.9$ & $4.6 \pm 1.8$ \\
\hline Hoehn \& Yahr severity & $1.63 \pm 0.8$ & $2.00 \pm 0.7$ & $2.50 \pm 0.4$ \\
\hline Daily CL dose (mg) & $350.00 \pm 57.7$ & $650.0 \pm 385.4$ & $825.00 \pm 330.4$ \\
\hline
\end{tabular}

CL, carbidopa-levodopa; PD, Parkinson’s disease; kg, kilograms; m, meters; mg, milligrams. Reprinted with permission from the Journal of Frailty and Aging. 
Table 7.2. Frailty Severity and Age- and Disease-Related Characteristics

Correlation to Frailty Severity $\quad \mathrm{P}$ value $\quad$ Beta Coefficient $\quad \mathrm{P}$ value

\begin{tabular}{lcccc}
\hline Non-PD Females: & & & & \\
Age (years) & 0.60 & 0.01 & 0.70 & 0.001 \\
BMI $\left(\mathrm{kg} / \mathrm{m}^{2}\right.$ ) & 0.38 & 0.13 & & 0.86 \\
Number of medications & 0.82 & 0.00 & 0.34 & 0.01 \\
Number of comorbidities & 0.57 & 0.01 & \\
\hline PD Females: & & & \\
Age (years) & -0.32 & 0.23 & \\
BMI (kg/m ${ }^{2}$ ) & 0.07 & 0.81 & \\
Number of medications & -0.22 & 0.42 & \\
Number of comorbidities & -0.32 & 0.23 & \\
Disease duration (years) & 0.32 & 0.23 & \\
Hoehn \& Yahr & 0.45 & 0.08 & \\
Daily CL dose (mg) & 0.61 & 0.01 & \\
\end{tabular}

CL, carbidopa-levodopa; $\mathrm{PD}=$ Parkinson's disease; $\mathrm{kg}=$ kilograms; $\mathrm{m}$ = meters; $\mathrm{mg}=$ milligrams; $\mathrm{p}$ value = significance. Reprinted with permission from the Journal of Frailty and Aging. 

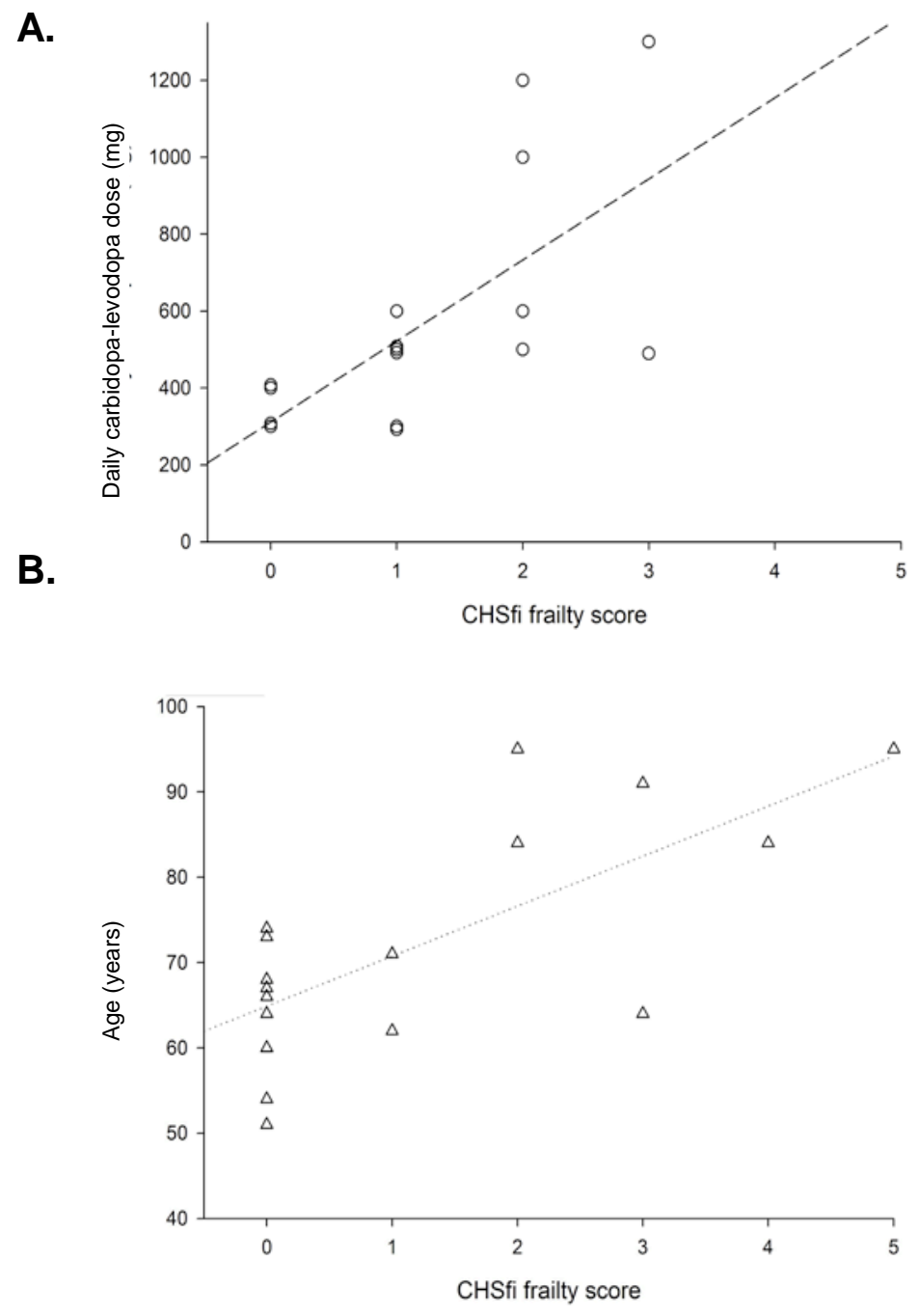

C.

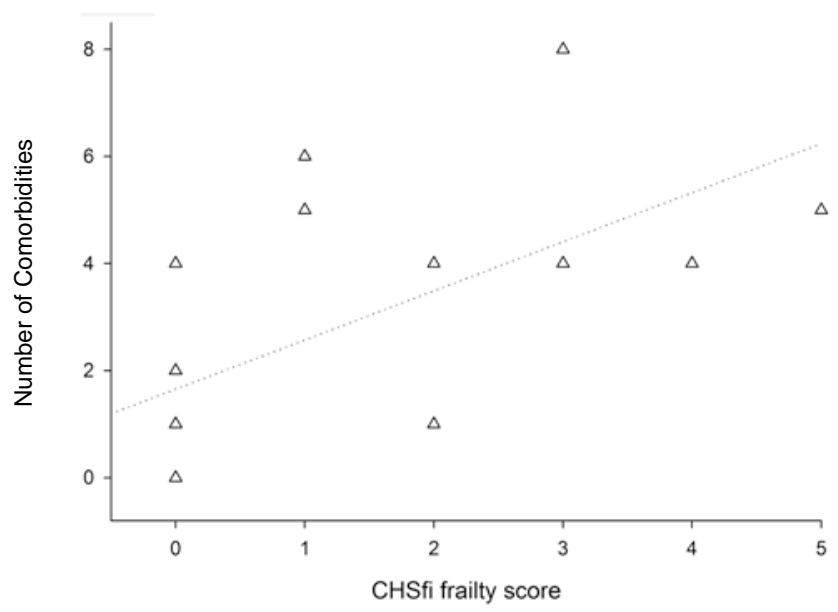

Figure 7.1. Relationship Between Frailty and Age- and Disease-Related Characteristics

Greater frailty severity and daily carbidopa-levodopa dose in females with PD (A) $(r=0.61)$. In females without PD, relationship between frailty and $(\mathbf{B})$ Age $(r=0.74)$ and $(\mathbf{C})$ Comorbidity $(r=0.54)$. Females with $\mathrm{PD}=$ open circles and dashed lines; females without PD = open triangles and dotted lines. Reprinted with permission from the Journal of Frailty and Aging. 


\subsection{Discussion}

Results of the current study demonstrate the characteristic that best explained frailty was daily carbidopa-levodopa dose $(\beta=0.50)$ in this sample of females with PD. This study suggests measures of disease progression (i.e., severity, duration) were not associated with frailty. Age ( $\beta$ $=0.70)$ and number of comorbidities $(\beta=0.47)$ best explained frailty in females without PD. These findings highlight the heterogeneous process of PD and indicate that dopaminergic management of PD symptoms may better reflect frailty in females with PD than disease severity and duration.

\subsubsection{Carbidopa-Levodopa Use Indicates Frailty}

Greater daily carbidopa-levodopa dosage is associated with frailty in this investigation of females with PD. The management of PD requires a multidisciplinary team approach, in which medication therapy plays an important role. Due to the progressive nature of PD, carbidopalevodopa dosage is adjusted to maintain physical function; this medication change may reflect symptom progression (i.e., exhaustion, rigidity) and/or resulting side effects of long-term use (i.e., gastrointestinal, wearing-off, dyskinesias). Physical exhaustion is cited as one of the most disabling symptoms among persons with PD and may be partly attributed to dopamine deficiency in PD (Grace et al., 2007). Current evidence suggests carbidopa-levodopa medication reduces exhaustion (Lou et al., 2003). Exhaustion has previously been reported as the strongest CHSfi frailty criteria to predict frailty in PD (Roland et al., 2012). Therefore, increased carbidopa-levodopa dose is prescribed to manage exhaustion, which is a criterion for frailty. This provides some interpretation as to why daily carbidopa-levodopa medication best explained frailty phenotype in our sample of females with PD. 


\subsubsection{Age and Frailty}

Accumulation of deficits that occur with age result in impaired physical capacity, and thus greater risk of frailty and mortality (Mitnitski, Mogilner, \& Rockwood, 2001). However, the current study findings suggest age-related deficits are not likely contributors to frailty within the context of individual disease states. This is evident since age was not a significant determinant of frailty in females with PD. Persons with PD seek long-term care sooner than those without PD (Aarsland et al., 2000). We speculate that similar to non-PD, older females with PD (i.e. > 80 years) are more likely to be frail and this population is likely no longer living in the community, and, therefore were not available for our sample.

\subsubsection{Is Comorbidity Masked by Parkinson’s Disease?}

Comorbidity may not be a significant contributor to frailty in females with PD since PD may mask other comorbidities. Although comorbidity impacts both mortality and disability in PD, it is rarely investigated. Common comorbidities within aging populations, such as cardiovascular disorders (Ciccone, Allegra, Cochrane, Cody, \& Roche, 1998) and arthritis (Sri Cherukumilli \& Kavanaugh, 2011), may be deprioritized due to the treatment focus placed on PD-symptom management. Therefore, a limitation to self-report is that persons with PD may be unaware that they have other comorbidities. Persons with PD are likely to report gastrointestinal and musculoskeletal problems; however, these are often not considered separate comorbidities because they are related to PD progression (Visser, Marinus, van Hilten, Schipper, \& Stiggelbout, 2004). In PD, comorbidity typically consists of disease-related symptoms or medication-induced side effects, such as psychosis, hallucinations, daytime sleepiness, depression, fatigue and dementia (Miyasaki, Shannon, \& Voon, 2006). In this study, females with PD reported significantly less comorbidity (non PD-related) than controls because the attention given to PD management may mask other comorbidities. Therefore, self-reported comorbidities from persons with PD may be biased by unrecognized comorbidity. 
Results demonstrate frailty severity increased with greater number of comorbidities and comorbidity explained frailty in non-PD females. Comorbidity is prevalent in communitydwelling older adults and increases risk of frailty through inactivity and loss of reserves (i.e., muscle weakness) across multiple physiologic systems (Fried et al., 2004). Multiple comorbidities are managed in older adults with polypharmacy, often prescribed from a variety of healthcare professionals. This can increase the risk of adverse drug reactions, resulting in functional impairment (i.e., falls, delirium) (Hilmer \& Gnjidic, 2009) and increased risk of frailty (Edelberg et al., 2000). Thus, comorbidity should be managed in the context of the whole individual (i.e., in association with other morbidities) to prevent further functional decline associated with mismanagement. It can be speculated that the number of comorbidities and related medication prescriptions may provide critical information regarding capacity for independent living and risk of transitioning to greater frailty (Edelberg et al., 2000) for homecare providers (i.e., community occupational and physical therapists). This may enable early identification of those at-risk for frailty and inform better decision-making practices regarding therapeutic management of functional outcomes and frailty.

\subsubsection{Frailty and Disease: Overlapping Concepts and Measures?}

In clinical practice, the presence of frailty may be misinterpreted as increasing PD severity (Powell, 2008). It is important to evaluate frailty in itself since the functional decline associated with frailty can be ameliorated through rehabilitative programs, namely exercise interventions (Theou, Stathokostas, Roland, Jakobi, Patterson, Vandervoort, \& Jones, 2011b). Ahmed and colleagues (2008) found that patients with PD have a five-fold higher prevalence of frailty than the general older adult population. The use of independent living subjects and a female-only sample adds strength to this study by removing the interaction of sex (i.e., male vs. female) and including a population where frailty detection is low but prevalence is high (i.e., community-dwelling). 
This study emphasizes the importance of addressing frailty within the context of PD. The presence of interacting clinical symptoms between frailty and PD in community-dwelling populations can make identification difficult for health professionals (Gobbens et al., 2010). CHSfi criteria are conceptually close to how PD is expressed; yet there is limited information as to whether frailty measures can distinguish declines in physical function as a result of PD or the consequence of frailty in persons with PD. For example, slowed walk-time could be due to ageassociated weakened leg strength (Inkster et al., 2003) or PD-associated bradykinetic movements resulting from decreased internal cues from the basal ganglia (Morris, Iansek, Matyas, \& Summers, 1994). Hence, PD may exaggerate the physical frail constitution. We suggest future studies of frailty consider community-dwelling populations that may be challenged with chronic disease or other comorbidities, which are typically exclusion criteria.

This study evaluated the associations between frailty and age- and disease-associated characteristics in a small sample of females with and without PD. Increased age places the older adult at greater risk for frailty (Fried et al., 2001). However age-related characteristics (including age, number of medication and number of comorbidities) were not clearly associated with frailty in this investigation of females with PD. Limitations of the sample size and cross-sectional nature of this study are acknowledged. To ensure generalization across a broad PD population future study should include a larger sample of persons living with PD at all stages of disease and frailty progression. Although this data supports the relationship between carbidopa-levodopa dose and frailty, this investigation does not allow for the determination of cause of frailty in PD. Further longitudinal work evaluating causal pathways for frailty and the role of dopaminergic therapy in moderating frailty in concert with PD symptoms is necessary. 


\subsection{Final Remarks}

Addressing frailty in PD is important, especially in females who are at greater risk for frailty and functional decline. In females with PD, disease severity does not reveal frailty, but rather daily carbidopa-levodopa dose best explains frailty by indicating declining symptomcontrol and the resulting negative impact on physical function. Interacting co-morbidities and clinical symptoms makes frailty identification challenging in practice. It is important to address the complexity of frailty within the context of PD. Understanding how frailty relates to PD progression may improve identification and early implementation of management strategies aimed at improving patient care and preserving independence. 


\section{Chapter 8: Discussion and Conclusions}

The purpose of this chapter is to:

1. Summarize the novel contributions and strengths of this dissertation research;

2. Address the limitations of this dissertation research and identify future research directions; and

3. Discuss overall implications directly related to supporting independent living in males and females with Parkinson's disease (PD).

\subsection{Novel Contributions to Scientific Knowledge}

The research presented within this dissertation contributes substantively to the existing scientific literature by: (a) Highlighting sex differences in the expression of PD; (b) Determining patterns of muscle quiescence attributed to PD; (c) Detecting frailty as separate from PD using muscle activation patterns; and (d) Identifying PD-specific frailty factors. The contributions of this work and its influence on daily physical function in persons with PD are discussed in greater detail in the following sections.

\subsubsection{Females with Parkinson’s Disease Experience Greater Functional Decline}

Results from the systematic review in Chapter 2 suggest that females with PD have different gait patterns, more freezing of gait, reduced force generation, poor balance control and lower exercise tolerance compared with age-matched healthy females and males with PD (Roland et al., 2011b). These differences acknowledge that females with PD are at greater risk for falls, fractures and functional dependence (Hughes et al., 2001; Taggart \& Crawford, 1999; Haaxma et al., 2007), although only a small portion of the PD literature considers the implications of PD on females. 


\subsubsection{Daily Muscle Quiescence and Strength Differs in Parkinson's Disease}

Chapter 3 determined EMG as a valuable assessment tool to examine upper and lower body muscle quiescence in persons with PD as they went about their routine daily activities. Todate, muscle activation patterns in PD have only been measured in a controlled laboratory environment, which arguably has limited transfer to the real world. This research presents new knowledge that muscles are less quiescent during routine daily activities and handgrip strength is reduced in persons with PD compared to age- and sex-matched controls. The results also indicate that females exhibited fewer periods of muscle quiescence and were weaker compared to males with PD. This is the first study to demonstrate sex differences associated with daily muscle quiescence in persons with PD. This research is important since it suggests EMG may identify underlying decline in physiological capacity before functional deficits are observed, especially in females with PD. In addition, bilateral asymmetrical differences in EMG characteristics and strength within persons with PD are also acknowledged. Specifically, less muscle quiescence was evident on the more affected compared with less affected side of persons with PD. The more affected side was weaker than the less affected side and persons with PD were weaker than ageand sex-matched controls.

This new knowledge helps to explain how declines in physiological capacity (i.e., decreased muscle quiescence) might contribute to functional limitations in PD. For example, dopamine degeneration results in decreased periods of muscle quiescence that indicate greater muscle fatigue. A consequence of muscle fatigue is the necessity to work at higher functional thresholds, resulting in greater incidence of self-reported exhaustion. Determining EMG thresholds required for independent living would inform practice by providing indicators of change that may identify subgroups at very low or high risk of becoming functionally dependent. Early diagnosis of frailty would allow for more timely and appropriate strategies to sustain physical independence, despite disease progression. Thus, from a prehabilitative perspective, the 
assessment of neuromuscular activity can provide critical information that may be used to avoid the vicious downward spiral of muscle weakness, inactivity, and eventual functional dependence (Speelman et al., 2011).

\subsubsection{Muscle Activation Differs with Frailty in Females with Parkinson's Disease}

A previous study demonstrated that decreased muscle activity and increased muscle quiescence reflects greater stages of frailty in older females (Theou et al., 2010). Conversely, the results in Chapter $\mathbf{4}$ present increased muscle activity and decreased muscle quiescence in frail compared with nonfrail females with PD. This dissertation is the first to investigate whether muscular activation patterns have the sensitivity to signal impending frailty in females with PD. These data not only indicate sensitivity, but dissociations can be made between nonfrail and frail through characterization of muscle activity in a population with an underlying neurological condition. The neuromuscular changes associated with frailty exacerbate the declines in physiological capacity associated with PD. The muscle activation patterns measured during a typical day suggest greater muscle fatigue, slower movement speed, and balance difficulties in frail females with PD, despite maintenance of physical activity levels across frailty phenotypes. Application of this knowledge lies in improving early identification of changes in muscle activation patterns that indicate impending frailty, to facilitate earlier intervention as well as inform targeted exercise programming specific to frail females with PD.

\subsubsection{Physical Activity Participation and Frailty in Females with Parkinson's Disease}

Adults living with chronic disease and/or physical disabilities are at greater risk of secondary de-conditioning, causing further and more rapid physical deterioration (Durstine, Painter, Franklin, Morgan, Pitetti, Roberts, 2000; Rimmer, 2005). Decreased levels of physical activity are also a key indicator of frailty (Rothman et al., 2008; Theou et al., 2011b). Chapter 5 reveals new information by demonstrating greater physical activity participation is associated with decreased frailty severity in females without PD; however, in the presence of PD, disease 
management is a better indicator of frailty. In this research, females with PD reported increased leisure time physical activity participation compared with sex- and age-matched controls. It could be speculated that persons with PD may be involved in more physical activity and better adhere to exercise recommendations compared with independent-living counterparts, regardless of frailty status, since the benefits of exercise are well recognized and encouraged within the PD population (Morey et al., 2002). There is a need to better understand the motivation to exercise in persons with $\mathrm{PD}$, since it may inform the modification of barriers and facilitate the development of long-term support for habitual physical activity in other populations living with chronic disease (Melillo, Williamson, Futrell \& Chamberlain, 1997).

\subsubsection{Quality of Life}

Declines in physical function and overall QoL are associated with PD progression (Chrischilles, Rubenstein, Voelker, Wallace, \& Rodnitzky, 1998). The results presented in Chapter 6 makes a significant contribution towards the identification of frailty within PD. The cumulative effect of PD and frailty is associated with lower QoL in persons with PD, especially females. This research is novel in that it demonstrates females with PD are 10 times more vulnerable to prefrailty than males with PD. In all persons with PD, mobility-related QoL was the specific domain that had the greatest association with frailty. Since CHSfi is a measure of physical ability, this emphasizes that QoL is greatly impacted by physical function in PD (Martinez-Martin, 1998). Understanding the relationship between QoL and frailty in the context of PD is important, because it will inform patient management strategies that meet the individual care needs of males and females with PD and ensure well-being throughout disease progression.

\subsubsection{Parkinson’s Disease Symptom Management and Frailty}

Progressive PD symptoms are managed with dopaminergic therapy. However, there is a wearing-off of drug efficacy resulting in dyskinesia and motor fluctuations (Lang \& Lees, 2002). Increasing motor complications, especially levodopa-induced dyskinesia, are related to 
reductions in QoL in persons with PD (Chapuis et al., 2004). Chapter 7 contributes new knowledge that suggests increased carbidopa-levodopa dosage relates to greater frailty severity because it reflects dopamine-related exhaustion and the need for greater symptom management. As PD severity increases, this may aggravate motor symptoms and exacerbate underlying frailty (Lauretani et al., 2012).

\subsection{Overall Conclusions}

In light of the current research in the field, the conclusions of this dissertation can be summarized as: Persons with PD are at increased risk for frailty and functional dependence during independent daily living. This increased risk is most evident in females who experience substantial changes in muscle activation patterns (a measure of physiological capacity) that will impact physical function and QoL. The implications of this research are that declines in physiological capacity combined with symptom expression make it challenging for persons with PD to complete tasks during daily life. Also, sex-differences in muscle activation and strength may be the predisposing factor to greater declines in physical function in females with PD. Muscle activation patterns can uniquely identify frailty in females with PD, and these frailty-related changes in muscle activation patterns may exacerbate the declines in physiological capacity. Factors attributed to these frailty-related declines are unique to PD. Physical inactivity may not be the primary factor associated with frailty in PD; however, it is proposed that disease progression leads to increased exhaustion and greater symptom management, resulting in greater frailty. Overall, the significance of this body of research is that it emphasizes sex differences in PD expression, measures physical and muscular activity that contributes to functional decline during independent real-life activities, and identifies relationships between PD-related factors (i.e., QoL, physical activity, disease characteristics) and frailty (Figure 8.1). 
Specific to each chapter:

- Chapter 2: Females with PD experienced greater declines in physical function compared with males;

- Chapter 3: Persons with PD, especially females, had less daily muscle quiescence compared with controls, indicating a greater decline in physiological capacity;

- Chapter 4: Frail females with PD had greater daily muscle activity and less quiescence compared with nonfrail;

- Chapter 5: Females with PD participated in more daily physical activity compared with non-PD females although a relationship between physical activity and frailty was not observed;

- Chapter 6: Females with PD were at greater risk for prefrailty compared to males. Increased exhaustion and decreased QoL, especially mobility-related, was associated with increased frailty;

- Chapter 7: Increased daily dopamine dosage was associated with greater frailty severity in males and females with PD. 


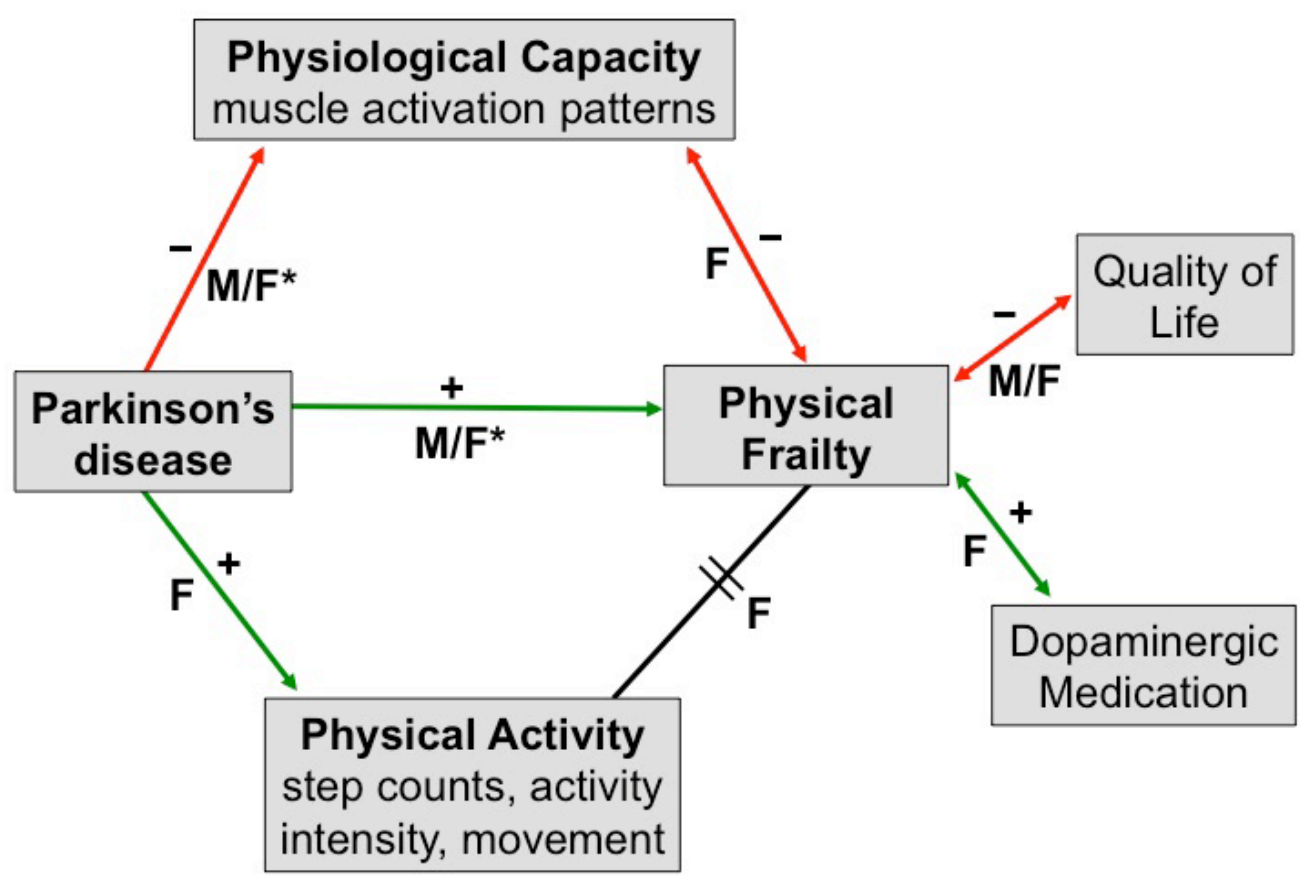

Figure 8.1. Physical Function and Quality of Life Outcomes in Parkinson's Disease.

Declines in physiological capacity, quality of life and greater symptom management were associated with greater frailty in persons with PD, especially females. -, negative relationship; +, positive relationship; $॥$, no relationship; $\mathrm{M} / \mathrm{F}^{*}$, males and especially females; $\mathrm{F}$ females only.

\subsection{Limitations and Future Research Directions}

This dissertation is unique in the fact that it considers sex differences, analyzes muscle activation patterns and physical activity participation while the subject goes about their normal daily routine (beyond laboratory- or clinical-based studies). Another novel contribution of this research is that it assesses frailty in persons with PD, who have often been omitted from frailty research. The strength of this research lies in the use of subjects living independently in their own home and consideration for sex differences. This enables the interaction of sex (i.e., male vs. female) to be elucidated and includes a population where frailty detection is low but prevalence is high. 
In spite of the contributions of this work highlighted above, it is necessary to reflect upon limitations inherent within this dissertation and address areas for future investigation. The research findings presented in this dissertation are applicable to future work aimed at: (a) Elucidation of sex and gender differences in PD expression; (b) Use of EMG as a clinical assessment tool; (c) Identification of frailty in PD and determination of causal factors; and (d) Development of exercise and rehabilitation guidelines specific to PD in the context of frailty and females. Specific to this dissertation, limitations are addressed and recommendations for future research directions in the field are outlined in the following sections.

\subsubsection{Sex Differences in Study Design}

Future studies should examine the potential for sex differences and consider improved methodology; primarily sex-matched recruitment with randomized allocation and blinding outcome evaluators to remove bias. The review presented in Chapter 2 acknowledges that only a small portion of the PD literature considers disease implications for females, thus, one might question if the available PD evidence is truly generalizable to females. Higher-powered studies with equal proportions of male and female subjects would enable more conclusive statements relative to sex differences associated with functional limitations and the contribution of female physiological capacity to symptom expression in PD. In addition, these results may reflect significant age differences between males and females with PD, as females are typically older (Twelves et al., 2003). Finally, this dissertation only investigated the biological construct of sex; however, one must acknowledge that gender influences also play a meaningful role in daily function of persons with PD. Gender analysis was beyond the scope of this thesis. Traditional societal roles often involve men and women completing different routine daily activities, which impact what PD symptoms mean to a person and how they contribute to QoL (Solimeo, 2008). Current evidence suggests gender influences are important in PD (Shulman 2007; Scott et al., 2000), and this needs to be further elucidated in light of physical function during daily life. 


\subsubsection{Muscle Activation Patterns in Parkinson's Disease}

The results presented in Chapters $\mathbf{3}$ and $\mathbf{4}$ propose that EMG patterns of muscle activity and quiescence may be an early indicator of PD-related functional decline and frailty. Limitations are acknowledged in portable research equipment used outside the laboratory during daily living. Specifically, in 17 subjects (41\% malfunction), the portable EMG devices (DataLog MX11506 \& MX11507, Biometrics Ltd.) were not able to retain enough battery power to save the daily recording file at the end of the $\sim 6.5$ hour testing day. These devices contained Bluetooth technology and were designed by the manufacturer to save the entire recording file after the daily collection was complete. However, replacing these devices with a previous model (DataLog P3X8, Biometrics Ltd.), without Bluetooth and that continuously saved the data as it was collected, resulted in 100\% success rate for the remaining 6 subjects. Previous investigation in our laboratory using this older model (DataLog P3X8, Biometrics Ltd.) demonstrated 97\% success rate during daily testing in various populations (i.e., young, old, frail, stroke) (Harwood et al., 2011; Jakobi et al., 2008; Theou et al., 2010; Theou et al., 2011). This demonstrates portable EMG is useful for capturing daily muscle activation patterns and most reliable when there is continuous file saving and minimal interaction with the environment (eg., Bluetooth).

The strength in encouraging subjects to go about their typical day (uncontrolled) is that it truly represents physiological capacity to remain independent in persons with PD; this cannot be captured in a controlled laboratory environment. However, it is recognized that muscle patterns and control processes vary for different types of contractions used to perform various tasks (i.e., anisometric vs. isometric contractions; Harwood et al, 2008). Therefore, it is suggested that future research explore which functional tasks (i.e., gait, balance, strength) best represents muscle activation patterns typical of daily life. This would better inform clinical assessments and provide an indication of physiological capacity (muscle activation patterns) required for specific tasks associated with independent living. 
Evidence suggests the rate of force generation and strength are influenced by dopaminergic medication in persons with PD; specifically, medication withdrawal results in further weakness compared to controls (Corcos et al., 1996). However, dose response relationship with carbidopa-levodopa and daily muscle activation patterns needs to be ascertained in future longitudinal investigations. Finally, long-term studies should investigate thresholds of change in muscle activation patterns that indicate impending frailty and PD progression. This information will further enable EMG to be used as a viable clinical assessment tool to detect patterns predictive of declining physiological capacity, functional dependence and frailty.

\subsubsection{Frailty Assessment in Parkinson's Disease}

This research provides new information about the relationship between frailty and PD in males and females. The impact of frailty on daily function suggests the need for greater awareness of frailty within the PD population. A variety of frailty assessment tools exist and there is no consensus on which criteria best identify frailty. This dissertation evaluated frailty using the CHSfi (Fried et al., 2001) because of its ability to capture physical characteristics associated with prefrailty. Due to its ease of application, the CHSfi is most applicable for clinical or community-based assessment of frailty; however, the CHSfi is not without its own limitations (Rockwood \& Mitnitski, 2007). Specifically, the CHSfi is considered unidimensional, and inclusion of additional social and psychological factors may provide greater insight to frailty development (Andrew, Mitnitski, \& Rockwood, 2008; Woo et al., 2005). It is hoped that this research encourages further investigation into the links between frailty and other neurological disorders that share commonalities related to decline in physical function. 


\subsubsection{Causal Factors Related to Frailty in Parkinson's Disease}

The limitations in assessing relationships between variables with cross-sectional data are discussed within Chapters 5 to 7. A limitation from these works is the causal relationship between PD-specific factors and frailty is unclear from the cross-sectional data. Further longitudinal cohort studies are recommended to evaluate whether frailty precedes, accompanies or follows the diagnosis of PD. This would help determine shared pathology and link thresholds of clinical symptoms in PD (i.e. disability resulting in transition to dependence) to frailty outcomes at different disease time points. These results, nonetheless, support further investigation into the impact of various stages of disease progression on frailty in males and females with PD. Also, since frailty is reversible, elucidating symptoms attributed to frailty would enable targeted interventions (i.e. exercise) so that improvements in function (resulting from decreased frailty) in persons with PD can be achieved. Future intervention studies need to focus on modifying factors related to QoL, frailty and PD-symptom management to improve patient-specific care.

\subsubsection{Exercise and Rehabilitation Guidelines}

Despite the known benefits of regular physical activity in the PD population (Chapter 5), it is apparent that barriers to participation still exist (Elsworth et al., 2009). Factors that enhance or impede physical activity participation in persons with PD, especially females, need to be further clarified to enable healthcare practitioners to modify perceived barriers and maximize perceived benefits. Additionally, there is a need to understand how the positive impact of exercise on frailty (Theou et al., 2011b) can be used effectively in the PD population. Exercise may improve physiological capacity and delay declines in physical function related to frailty, thus can play a profound role in managing PD day-to-day. Results from this dissertation suggest optimization of rehabilitation needs to consider various clinical expressions of PD, especially in the context of frailty (Chapter 4-7) and being female (Chapter 2). Also, relevant to this 
research, long-term studies should investigate the continued benefit of exercise on neuromuscular function (Chapter 3-4) and rate of PD progression (David et al., 2012). A rigorous rehabilitation and exercise program that focuses on anticipated declines, which are inevitable with PD progression, may help patients who do not yet exhibit mobility problems and functional dependence (King \& Horak, 2009). Prehabilitation strategies have the potential to reduce physical, emotional, and social problems attributable to declining function, improve QoL in persons with PD (Chapter 6) and generate large health care savings (Gill et al., 2002; Guralnik, Alecxih, Branch, \& Wiener, 2002). Specifically, two aspects of rehabilitation optimization that need to be evaluated in persons with PD are: (1) Specification of the dimensions of exercise (i.e., frequency, intensity, time, and type); and (2) Determining the most effective mode of exercise (David et al., 2012). Knowledge gained from this research contributes valuable information on physiological capacity of persons with PD, especially frail females, which would help direct exercise programming guidelines.

\subsection{Implications}

This dissertation represents the first body of research to assess physiological capacity during independent daily living and determine PD-specific contributors to frailty. Knowledge gained contributes novel findings towards timely identification of functional decline in males

and females with PD. Future longitudinal studies are required to establish meaningful changes in contributors to frailty (i.e., QoL, dopaminergic therapy) with respect to PD progression. At the conclusion of this doctoral experience, I have a comprehensive understanding of defining features of PD in females compared to males and understand the impact of PD on physiological capacity, independent daily living, and physical function. This research represents the integration of physiology (i.e., EMG, daily physical activity participation) and health research (i.e., QoL, PD-specific factors associated with frailty) and is intended to influence relevant assessment, 
treatment and rehabilitation pathways for the management of PD, especially females. In the future, understanding physiological capacity (muscle activation patterns), physical activity participation and physical function (frailty) may support appropriate rehabilitation strategies and symptom management, enabling healthcare providers to address the needs of their patients in a more timely and appropriate manner. Ultimately, this new knowledge will enable persons with PD to remain active participants in their community, as well as advocate for programs and services that are needed to maintain their independence through enhancing physiological capacity. 


\section{References}

Aarsland, D., Larsen, J.P., Tandberg, E., \& Laake, K. (2000). Predictors of nursing home placement in Parkinson's disease: a population-based, prospective study. Journal of the American Geriatrics Society, 48(8), 938-942.

Ahmed, N.N., Sherman, S.J., \& VanWyck, D. (2008). Frailty in Parkinson's disease and its clinical implications. Parkinsonism and Related Disorders, 14, 334-337.

Allen, D.G., Lamb, G.D., \& Westerblad, H. (2008). Skeletal muscle fatigue: Cellular mechanisms. Physiology Reviews, 88(1), 287-332.

Andrew, M.K., Mitnitski, A.B., \& Rockwood, K. (2008). Social vulnerability, frailty and mortality in elderly people. PLoS ONE, 3(5), e2232.

Ashburn, A., Stack, E., Pickering, R.M., \& Ward, C.D. (2001). A community-dwelling sample of people with Parkinson's disease: Characteristics of fallers and non-fallers. Age and Ageing, 30(1), 47-52.

Avila-Funes, J.A., Amieva, H., Barberger-Gateau, P., LeGoff, M., Raoux, N., Ritchie, K. ... Dartigue, J.F. (2009). Cognitive impairment improves the predictive validity of the phenotype of frailty for adverse health outcomes: The three-city study. Journal of the American Geriatrics Society, 57(3), 453-461.

Avlund, K., Kreiner, S., \& Schultz-Larsen, K. (1996). Functional ability scales for the elderly. European Journal of Public Health, 6(1), 35-42.

Baba, Y., Putzke, J.D., Whaley, N.R., Wszolek, Z.K., \& Utti, R.J. (2005). Gender and the Parkinson's disease phenotype. Journal of Neurology, 252, 1201-1205.

Baker, P.S., Bodner, E.V., \& Allman, R.M. (2003). Measuring life space mobility in communitydwelling older adults. Journal of the American Geriatrics Society, 51, 1610-1614.

Baldereschi, M., DiCarlo, A., Rocca, W.A., Vanni, P., Maggi, S., Perissinotto, E. ... Inzitari, D. (2000). Parkinson's disease and parkinsonism in a longitudinal study: Two fold higher incidence in men. Neurology, 55(9), 1358-1363.

Bales, C.W. \& Ritchie, C.S. (2002). Sarcopenia, weight loss, and nutritional frailty in the elderly. Annual Reviews in Nutrition, 22, 309-323.

Bandeen-Roche, K., Xue, Q.L., Ferruci, L., Walston, J., Guralnik, J.M., Chaves, P. ... Fried, L.P. (2006). Phenotype of frailty: Characterization in the women's health and aging studies. Journal of Gerontology A Biological Sciences Medical Sciences, 61(3), 262-266.

Behari, M., Srivastava, A.K., \& Pandey, R.M. (2005). Quality of life in patients with Parkinson’s disease. Parkinsonism and Related Disorders, 11, 221-226.

Ben Shlomo, Y. (1997). The epidemiology of Parkinson’s disease. Baillieres- Clinical Neurology, 6(1), 55-68.

Bergman, H., Béland, F., Karunanathan, S., Hummel, S., Hogan, D., \& Wolfson, C. (2004). Developing a working framework for understanding frailty. Gérontologie et Société, 109, 15-29.

Berkman, L.F., Seeman, T.F., \& Albert, N.I. (1993). High, usual and impaired functioning in community-dwelling older men and women: Findings from the MacArthur Foundation Research Network on Successful Aging. Journal of Clinical Epidemiology 46, 1129-1140. 
Blangsted, A.K., Hansen, K., \& Jensen, C. (2003). Muscle activity during computer-based office work in relation to self-reported job demands and gender. European Journal of Applied Physiology, 89, 352-358.

Bloem, B.R., Grimbergen, Y.A.M., Cramer, M., Willemsen, M., \& Zwinderman, A.H. (2001). Prospective assessment of falls in Parkinson's disease. Journal of Neurology, 248, 950958.

Borges, L.L. \& Menezes, R.L. (2011). Definition and markers of frailty: A systemic review. Reviews in Clinical Gerontology, 21, 67-77.

Breit, S., Spieker, S., Schulz, J.B., \& Gasser, T. (2008). Long-term EMG recordings differentiate between parkinsonian and essential tremor. Journal of Neurology, 255, 103-111.

Brown, P., Corcos, D.M., \& Rothwell, J.C. (1998). Action tremor and weakness in Parkinson's disease: A study of the elbow extensors. Movement Disorders, 13, 56-60.

Brown, P., Corcos, D.M., \& Rothwell, J.C. (1997). Does parkinsonian action tremor contribute to muscle weakness in Parkinson's disease? Brain, 120, 401-408.

Brown, M., Sinacore, D.R., Binder, E.F., \& Kohrt, W.M. (2000). Physical and performance measures for the identification of mild to moderate frailty. Archives of Physical Medicine and Rehabilitation, 81(7), 960-965.

Brown, P. (2003). Oscillatory nature of human basal ganglia activity: relationship to the pathophysiology of Parkinson's disease. Movement Disorders, 18(4), 357-363.

Canadian Institute for Health Information (CIHI). (2007). The Burden of Neurological Diseases, Disorders and Injuries in Canada (Ottawa).

Candow, D.G. \& Chilibeck, P.D. (2005). Differences in size, strength, and power of upper and lower body muscle groups in young and older men. Journals of Gerontology A Biological Sciences Medical Sciences, 60, 148-156.

Chapuis, S., Ouchchane, L., Metz, O., Gerbaud, L., \& Durif, F. (2005). Impact of the motor complications of Parkinson's disease on quality of life. Parkinsonism and Related Disorders, 20(2), 224-230.

Chen, J.S., Lipska, B.K., Halim, N., Ma, Q.D., Matsumoto, M., Melhem, S. ... Weinberger, D.R. (2004). Functional analysis of genetic variation in catechol-O-methyltransferase (COMT): Effects on mRNA, protein, and enzyme activity in postmortem human brain. American Journal of Human Genetics, 75, 807-821.

Chrischilles, E.A., Rubenstein, L.R., Voelker, M.D., Wallace, R.B., \& Rodnitzky, R.L. (1998). The health burdens of Parkinson's disease. Movement Disorders, 13(3), 406-413.

Christou E.A., Jakobi J.M., Critchlou A., \& Enoka R.M. (2004). The 1 - 2 Hz oscillations in muscle force are exacerbated by stress, especially in older adults. Journal of Applied Physiology, 97(7), 225-236.

Ciccone, A., Allegra, J.R., Cochrane, D.G., Cody, R.P., \& Roche, L.M. (1998). Age-related differences in diagnoses within the elderly population. American Journal of Emergency Medicine, 16(1), 43-48.

Cigolle, C.T., Ofstedal, M.B., Tian, Z., \& Blaum, C.S. (2009). Comparing models of frailty: The health and retirement study. Journal of the American Geriatrics Society, 57(5), 830-839. 
Clark, D.J., Patten, C., Reid, K.F., Carabello, R.J., Phillips, E.M., \& Fielding, R.A. (2010). Impaired voluntary neuromuscular activation limits muscle power in mobility-limited older adults. Journal of Gerontology A Biological Sciences Medical Sciences, 65A, 495502.

Clark, D.J., Patten, C., Reid, K.F., Carabello, R.J., Phillips, E.M., \& Fielding, R.A. (2011). Muscle performance and physical function are associated with voluntary rate of neuromuscular activation in older adults. Journal of Gerontology A Biological Sciences Medical Sciences, 66A(1), 115-121.

Collier, T.J., Kanaan, N.M., \& Kordower, J.H. (2011). Ageing as a primary risk factor for Parkinson's disease: Evidence from studies of non-human primates. Nature Reviews Neuroscience, 12, 359-366.

Copeland, J.L. \& Esliger, D.W. (2009). Accelerometer assessment of physical activity in active, healthy older adults. Journal of Aging and Physical Activity, 17(1), 17-30.

Corcos, D.M., Chen, C.M., Quinn, N.P., McAuley, J., \& Rothwell, J.C. (1996). Strength in Parkinson's disease: Relationship to rate of force generation and clinical status. Annals of Neurology, 39, 79-88.

Crizzle, A.M. \& Newhouse, I.J. (2006). Is physical exercise beneficial for persons with Parkinson’s disease? Clinical Journal of Sports Medicine, 16, 422-425.

Damiano, A.M., Snyder, C., Strausser, B., \& William, M.K. (1999). A review of health-related quality-of-life concepts and measures for Parkinson's disease. Quality of Life Research, 8(3), 235-243.

David, F.J., Rafferty, M.R., Robichaud, J.A., Prodoehl, J., Kohrt, W.M., Vaillancourt, D.E., \& Corcos, D.M. (2012). Progressive resistance exercise and Parkinson’s disease: A review of potential mechanisms. Parkinson's disease, \#124527.

DeLong, M. \& Wichmann, T. (2010). Changing view of basal ganglia circuits and circuit disorders. Clinical EEG and Neuroscience, 41(2), 61-67.

Dibble, L.E., Addison, O., \& Papa, E. (2009). The effects of exercise on balance in persons with Parkinson's disease: A systematic review across the disability spectrum. Journal of Neurology and Physical Therapy. 33(1), 14-26.

Dibble, L.E., Hale, T.F., Marcus, R.L., Gerber, J.P., \& LaStayo, P.C. (2009). High intensity eccentric resistance training decreases bradykinesia and improves quality of life in persons with Parkinson's disease: A preliminary study. Parkinsonism and Related Disorders, 15(10), 752-757.

Dietz, V., Zijlstra, W., Prokop, T., \& Berger, W. (1995). Leg muscle activation during gait in Parkinson's disease: adaptation and interlimb coordination. Electroencephalography and Clinical Neurophysiology, 97(6), 408-415.

Dijkstra, B., Kamsma, Y., \& Zijlstra, W. (2010). Detection of gait and postures using a miniaturized triaxial accelerometer-based system: Accuracy in community-dwelling older adults. Age and Ageing, 39(2), 259-262.

Djaldetti, R., Ziv, I., \& Melamed, E. (2006). The mystery of motor asymmetry in Parkinson's disease. Lancet Neurology, 5(9), 796-802. 
Downs, S.H. \& Black, N. (1998). The feasibility of creating a checklist for the assessment of the methodological quality both of randomized and non-randomized studies of health care interventions. Journal of Epidemiology and Community Health, 52, 377-384.

Durstine, J.L., Painter, P., Franklin, B.A., Morgan, D., Pitetti, K.H., \& Roberts, S.O. (2000). Physical activity for the chronically ill and disabled. [erratum appears in Sports Medicine (2001) 31(8), 627]. Sports Medicine, 30, 207-219

Ebersback, G., Ebersback, A., Edler, D., Kaufhold, O., Kusch, M., Kupsch, A., \& Wissel, J. (2010). Comparing exercise in Parkinson’s disease - the Berlin LSVT®Big study. Movement Disorders, 25(12), 1902-1908.

Edelberg, H.K., Shallenberger, E., Hausdorff, J.M., \& Wei, J.Y. (2000). One-year follow-up of medication management capacity in highly functioning older adults. Journals of Gerontology A Biological Sciences Medical Sciences, 55(10), M550-M553.

Edstrom, L. (1970). Selective changes in the sizes of red and white muscle fibers in upper motor lesions and parkinsonism. Journal of Neurological Sciences, 11, 537-550.

Elsworth, C., Dawes, H., Sackley, C., Soundy, A., Howells, K., Wade, D. ... Izadi, H. (2009). A study of perceived facilitators to physical activity in neurological conditions. International Journal of Therapy and Rehabilitation, 16(1), 17-24.

Enoka, R.M. (1997). Neural adaptations with chronic physical activity. Journal of Biomechanics, 30, 447-455.

Erickson, K.I., Voss, M.W., Prakash, R.S., Basak, C., Szabo, A., Chaddock, L.... Kramer, A.F. (2011). Exercise training increases size of hippocampus and improves memory. Proc National Academy of Science USA, 108, 3017-3022.

Fallah, N., Mitnitski, A., Searle, S.D., Gahbauer, E.A., Gill, T.M., \& Rockwood, K. (2011). Transitions in frailty status in older adults in relation to mobility: A multistate modeling approach employing a deficit count. Journal of the American Geriatrics Society, 59, 524529.

Fellows, S.J., Noth, J., \& Schwarz, M. (1998). Precision grip and Parkinson’s disease. Brain, 121, 1771-1784.

Fertl, E., Doppelbauer, A., Auff, E. (1993). Physical activity and sport in patients suffering from Parkinson's disease in comparison with healthy seniors. Journal of Neural Transmission, 5, 157-161.

Fiatarone-Singh, M.A. (2002). Exercise comes of age: rationale and recommendations for a geriatric exercise prescription. Journal of Gerontology A Medical Sciences, 57(5), M262M282.

Fitzpatrick, R., Peto, V., Jenkinson, C., Greenhall, R., \& Hyman, N. (1997). Health-related quality of life in Parkinson's disease: A study of outpatient clinic attenders. Movement Disorders, 12(6), 916-922.

Fleming, V., Tolson, D., \& Schartau, E. (2004). Changing perceptions of womanhood: Living with Parkinson's disease. International Journal of Nursing Science, 41, 515-524.

Fox, C.M., Ramig, L.O., Ciucci, M.R., Sapir, S., McFarland, D.H., \& Farley, B.G. (2006). The science and practice of LSVT/LOUD: Neural plasticity principled approach to treating individuals with Parkinson's disease and other neurological disorders. Seminal Speech Language, 27, 283-299. 
Fried, L.P., Ettinger, W.H., Lind B., Newman, A.B., \& Gardin, J. (1994). Physical disability in older adults: a physiological approach. Journal of Clinical Epidemiology, 47, 747-760.

Fried, L.P., Ferrucci, L., Darer, J., Williamson, J.D., \& Anderson, G. (2004). Untangling the concepts of disability, frailty and comorbidity: Implication for improved targeting and care. Journal of Gerontology A Biological Sciences Medical Sciences, 59(3), 255-263.

Fried, L.P., Tangen, C.M., Walston, J., Newman, A.B., Hirsch, C., Gottdiener, J. ... Cardiovascular Health Study Collaborative Research Group. (2001). Frailty in older adults: Evidence for a phenotype. Journals of Gerontology A Biological Sciences Medical Sciences, 56(3), M146-M156.

Fujikane, M., Katayama, S., Hirata, K., \& Yokota, N. Objective measurement of motor activity in Alzheimer's disease and Parkinson's disease (1997). IEEE Transactions on Rehabilitation Engineering, 5(4), 394-398.

Galvan, A. \& Wichman, T. (2008). Pathophysiology of Parkinson’s. Clinical Neurophysiology, 119, 1459-1474.

Garber, C.E. \& Friedman, J.H. (2003). Effects of fatigue on physical activity and function in patients with Parkinson's disease. Neurology, 60(7), 1119-1124.

Gaspar, P., Duyckaerts, C., Alvarez, C., Javoy-Agid, F., \& Berger, B. (1991). Alterations of dopaminergic and noradrenergic innervations in motor cortex in Parkinson's disease. Annals of Neurology, 30, 365-374.

Geurts, A.C.G., Boonstra, T.A., Voermans, N.C., Dienders, M.G., Weerdesteyn, V., \& Bloem, B.R. (2011). Assessment of posture asymmetry in mild to moderate Parkinson's disease. Gait and Posture, 33(1), 143-145.

Gill, T.M., Baker, D.I., Gottschalk, M., Peduzzi, P.N., Allore, H., \& Byers, A. (2002). A program to prevent functional decline in physically frail, elderly persons who live at home. New England Journal of Medicine, 347, 1068-1074.

Gill, T.M., Gahbauer, E.A., Allore, H.G., \& Han, L. (2006). Transitions between frailty states among community-living older persons. Archives of Internal Medicine, 166(4), 418-423.

Glendinning, D. (1997). A rational for strength training in patients with Parkinson's disease. Neurological Reports, 21, 132-135.

Glendinning, D.S. \& Enoka, R.M. (1994). Motor unit behavior in Parkinson’s disease. Physical Therapy, 74, 61-70.

Gobbens, R.J.J., Luijkx, K.G., Wijnen-Sponselee, M.T., \& Schols, J.M.G.A. (2010). Towards an integral conceptual model of frailty. Journal of Nutrition, Health and Aging, 14(3), 175181.

Goodwin, V.A., Richards, S.H., Taylor, R.S., Taylor, A.H., \& Campbell, J.L. (2008). The effectiveness of exercise interventions for people with Parkinson's disease: A systematic review and meta-analysis. Movement Disorders, 23(5), 631-640.

Grace, J., Mendelsohn, A., \& Friedman, J.H. (2007). A comparison of fatigue measures in Parkinson’s disease. Parkinsonism and Related Disorders, 13, 443-445.

Grimbergen, Y.A.M., Munneke, M., \& Bloem, B.R. (2004). Falls in Parkinson’s disease. Current Opinion in Neurology, 17, 405-415. 
Guralnik, J.M., Ferrucci, L., Pieper, C.F., Leveille, S.G., Markides, K.S., Ostir, G.V. ... Wallace, R.B. (2000). Lower extremity function and subsequent disability. Journal of Gerontology A Biological Sciences Medical Sciences, 55(4), M221-M231.

Guralnik, J.M., Ferrucci, L., Simonsick, E.M., Salive, M.E., \& Wallace, R.B. (1995). Lower extremity function in persons over the age of 70 years as a predictor of subsequent disability. New England Journal of Medicine, 332, 556-561.

Guralnik, J.M., Alecxih, L., Branch, L.G., \& Wiener, J.M. (2002). Medical and long-term care costs when older persons become more dependent. American Journal of Public Health, 92, 1244-1245.

Haaxma, C.A., Bloem, B.R., Borm, G.F., Oyen, W.J.G., Leenders, K.L., Eshuis, S. ... Horstink, M.W. (2007). Gender differences in Parkinson's disease. Journal of Neurology, Neurosurgery and Psychiatry, 78, 819-824.

Hallett, M., \& Khoshbin, S. (1980). A physiological mechanism of bradykinesia. Brain, 103, 301-314.

Hammerman, D. (1999). Towards an understanding of frailty. Annuals of Internal Medicine, 130, 945-950.

Harwood, B., Edwards, D.L., \& Jakobi, J.M. (2008). Age and sex-related differences in muscle activation for a discrete functional task. European Journal of Applied Physiology, 103(6), 677-686.

Harwood, B., Edwards, D.L., \& Jakobi, J.M. (2011). Age- and sex-related differences for electromyography gaps during daily activity and a discrete task. Gait and Posture, 110(1), 27-38.

Herrmann, F.R., Osiek, A., Cos, M., Michael, J.P., \& Robine, J.M. (2005). Frailty judgment by hospital team members: Degree of achievement and survival prediction. Journal of the American Geriatrics Society, 53(5), 916-917.

Hilmer, S. \& Gnjidic, D. (2009). The effects of polypharmacy in older adults. Clinical Pharmacological Therapy, 85(1), 86-88.

Hirsch, H., Fried, L.P., Harris, T., Fitzpatrick, A., Enright P., \& Schulz, P. (1997). Correlates of performance-based measures of muscle function in the elderly: The Cardiovascular Health Study. Journal of Gerontology A Biological Sciences Medical Sciences, 52(4), M142-200.

Hirsch, M.A., Toole, T., Maitland, C.G., \& Rider, R.A. (2003). The effects of balance training and high-intensity resistance training on persons with idiopathic Parkinson's disease. Archives of Physical Medicine and Rehabilitation, 84, 1109-1117.

Hobson, P., Holden, A., \& Meara, J. (1999). Measuring the impact of Parkinson's disease with the Parkinson's disease quality of life questionnaire. Age and Ageing, 28(4), 341-346.

Hoehn, M.M. \& Yahr, M.D. (1967). Parkinsonism: Onset, progression, and mortality. Neurology, 17, 427-444.

Howe, T.E., \& Rafferty, D. (2009). Quadriceps activity and physical activity profiles over long durations in patients with osteoarthritis of the knee and controls. Journal of Electomyography and Kinesiology, 19, 78-83. 
Hughes, V.A., Frontera, W.R., Wood, M., Evans, W.J., Dallal, G.E., Roubenoff, R., \& Fiatrone Singh, M.A. (2001). Longitudinal muscle strength changes in older adults: Influence of muscle mass, physical activity and health. Journals of Gerontology A Biological Sciences, 56(5), B209-217.

Inkster, L.M., Eng, J.J., MacIntyre, D.L., \& Stoessl, A.J. (2003). Leg muscle strength is reduced in Parkinson's disease and related to the ability to rise from a chair. Movement Disorders, 18, 157-162.

Jakobi, J.M., Edwards, D.L., \& Connelly, D.M. (2008). Utility of portable electromyography for quantifying muscle activity during daily use. Gerontology, 54, 324-331.

Jankovic, J., \& Kapadia, A.S. (2001). Functional decline in Parkinson’s disease. Archives of Neurology, 58(10), 1611-1615.

Jankovic, J. (2008). Parkinson's disease: Clinical features and diagnosis. Journal of Neurology, Neurosurgery and Psychiatry, 79(4), 368-376.

Jenkinson, C., Fitzpatrick, R., Peto, V., Greenhall, R., \& Hyman, N. (1997). The Parkinson’s disease questionnaire (PDQ-39): Development and validation of a Parkinson's disease summary index score. Age and Ageing, 26, 353-357.

Johnson, J. L., Greaves, L., \& Repta, R. (2007). Better science with sex and gender: A primer for health research. Vancouver: Women’s Health Research Network.

Jong, L.T., Comella, C.L., \& Jaglin, J.A. (1995). Menstrual-related cyclic changes in women with Parkinson's disease. Neurology, 45, A337.

Jordan, N., Sagar, H.J., \& Cooper, J.A. (1992). A component analysis of the generation and release of isometric force in Parkinson's disease. Journal of Neurology, Neurosurgery and Psychiatry, 55, 572-576.

Kakinuma, S., Nogaki, H., Pramanik, B., \& Morimatsu, M. (1998). Muscle weakness in Parkinson's disease: Isokinetic study of the lower limbs. European Neurology, 39, 218222.

Karnik, K. \& Mazzatti, D.J. (2009). Review of tools and technologies to assess multi-system functional impairment and frailty. Clinical Medicine: Geriatrics, 3, 1-8.

Kent-Braun, J.A. (2009). Skeletal muscle fatigue in old age: Whose advantage? Exercise Sport Science Reviews, 37(1), 3-9.

Kent-Braun, J.A. \& Ng, A.V. (1999). Specific strength and voluntary muscle activation in young and elderly women and men. Journal of Applied Physiology, 87(1), 22-29.

Kern, D.S., Semmler, J.G., \& Enoka, R.M. (2001). Long-term activity in upper- and lower-limb muscles of humans. Journal of Applied Physiology, 91, 2224-2232.

Kimura, H., Kurimura, M., Wada, M., Kawanami, T., Kurita, K., Suzuki, Y. ... Kato, T. (2002). Female preponderance of Parkinson's disease in Japan. Neuroepidemiology, 21, 292-296.

King, L.A. \& Horak, F.B. (2009). Delaying mobility disability in people with Parkinson disease using a sensorimotor agility exercise program. Physical Therapy, 89(4), 384-393.

Kokko, S.M., Paltamaa, J., Ahola, E., \& Malkia, E. (1997). The assessment of functional ability in patients with Parkinson's disease: The PLM-test and three clinical tests. Physiotherapy Research International, 2(2), 29-45. 
Koller, W. \& Kase, S. (1986). Muscle strength testing in Parkinson's disease. European Neurology, 25, 130-133.

Kompoliti, K., Comella, C.L., Jaglin, J.A., Leurgans, S., Raman, R., \& Goetz, C.G. (2000). Menstrual-related changes in motoric function in women with Parkinson's disease. Neurology, 55(10), 1572-1575.

LaCroix, A.Z., Guralnik, J.M., Berkman, L.F., Wallace, R.B., \& Satterfield, S. (1993). Maintaining mobility in late life. II. Smoking, alcohol consumption, physical activity and body mass index. American Journal of Epidemiology, 137, 858-869.

Lafargue, G., D'Amico, A., Thobois, S., Broussolle, E., \& Sirigu, A. (2008). The ability to assess muscular force in asymmetrical Parkinson's disease. Cortex, 44(1), 82-89.

Lamberti, P., Armenise, S., Castaldo, V., deMari, M., Iliceto, G., Tronci, P., \& Serlenga, L. (1997). Freezing of gait in Parkinson's disease. European Neurology, 38, 297-301.

Lang, A.E. \& Lees, A. (2002). Management of Parkinson’s disease: An evidence-based review. Movement Disorders, 17(4), S1-S166.

Lauretani, F., Maggio, M., Silvestrini, C., Nardelli, A., Saccavini, M., \& Paolo Ceda, G. (2012). Parkinson's disease in the elderly: An example of geriatric syndrome (GS)? Archives of Gerontology and Geriatrics, 54, 242-246.

Laursen, B., Jensen, B.R., \& Ratkevicius, A. (2001). Performance and muscle activity during computer mouse tasks in young and elderly adults. European Journal of Applied Physiology, 84, 329-336.

Lawrence, R.H. \& Jette, A.M. (1996). Disentangling the disablement process. Journals of Gerontology B Psychological Science Social Science, 51B, S173-S182.

Lees, A.J., Hardy, J., \& Revesz, T. (2009). Parkinson’s disease. Lancet, 373, 2055-2066.

Leveille, S.G., Penninx, B.W., Melzer, D., Izmirlian, G., \& Guralnik, J.M. (2004). Sex differences in the prevalence of mobility disability in old age: The dynamics of incidence, recovery, and mortality. Journals of Gerontology B Psychological Science Social Science, 55(1), S41-S50.

Levers, M.J., Estabrooks, C.A., \& Ross Kerr, J.C. (2006). Factors contributing to frailty: Literature review. Journal of Advanced Nursing, 56(3), 282-291.

Lipsitz, L.A. (2004). Physiological complexity, aging, and the path to frailty. Scientific Aging Knowledge and Environment, 16, pe16.

Logigian, E., Hefter, H., Reiners, K., \& Freund, H.J. (1991). Does tremor pace repetitive voluntary motor behavior in Parkinson's disease? Annals of Neurology, 30, 172-179.

Lou, J.S., Kearns, G., Benice, T.B., Oken, B., Sexton, G., \& Nutt, J. (2003). Levodopa improves physical fatigue in Parkinson’s disease. Movement Disorders, 18(10), 1108-1114.

Lou, J.S., Kearns, G., Oken, B., Sexton, G., \& Nutt, J. (2001). Exacerbated physical and mental fatigue in Parkinson's disease. Movement Disorders, 16, 190-196.

Maggio, M., Cappola, A.R., Ceda, G.P., Basaria, S., Chia, C.W., Valenti, G., \& Ferrucci, L. (2005). The hormonal pathway to frailty in older men. Journal of Endocrinological Investigations, 28(S11), 15-19. 
Mak, M.K. \& Pang, M.Y. (2009). Balance confidence and functional mobility are independently associated with falls in people with Parkinson's disease. Journal of Neurology, 256(5), 742-749.

Martinez-Martin, P. (1998). An introduction to the concept of 'quality of life in Parkinson's disease'. Journal of Neurology, 245(S1), S2-S6.

Marusiaka, J., Jaskolska, A., Kisiel-Sajewicz, K., Yue, G.H., \& Jaskolski, A. (2009). EMG and MMG activities of agonist and antagonist muscles in Parkinson's disease patients during absolute submaximal load holding. Journal of Electromyography and Kinesiology, 19(5), 903-914.

Matteini, A.M., Walston, J.D., Fallin, M.D., Bandeen-Roche, K., Kao, W.H.L., Semba, R.D. ... Stabler, S.P. (2008). Markers of B-vitamin deficiency and frailty in older women. Journal of Nutrition, Health and Aging, 12(5), 303-308.

Melillo, K.D., Williamson, E., Futrell, M., \& Chamberlain, C. (1997). A self-assessment tool to measure older adult's perceptions regarding physical fitness and exercise activity. Journal of Advanced Nursing, 25, 1220-1226.

Miller, R.A., Thaut, M.H., McIntosh, C.G., \& Rice, R.R. (1996). Components of EMG symmetry and variability in Parkinsonian and healthy elderly gait. Electroencephalogram and Clinical Neurophysiology, 101(1), 1-7.

Miller, I.N. \& Cronin-Golomb, A. (2010). Gender differences in Parkinson’s disease: Clinical characteristics and cognition. Movement Disorders, 25(16), 2695-2703.

Mitnitski, A., Mogilner, A., \& Rockwood, K. (2001). Accumulation of deficits as a proxy measure of aging. Science World Journal, 1, 323-336.

Mitnitski, A.B., Song, X., \& Rockwood, K. (2004). The estimation of relative fitness and frailty in community-dwelling older adults using self-report data. Journals of Gerontology A Biological Science Medical Science, 59(6), M627-632.

Miyasaki, J.M., Shannon, K., \& Voon, V. (2006). Quality standards subcommittee of the American academy of neurology. practice parameter: Evaluation and treatment of depression, psychosis and dementia in Parkinson's disease (an evidence based review). Neurology, 66, 996.

Mor, V., Murphy, J., Masterson-Allen, S., Willey, C., Razmpour, A., Jackson, M.E., ... Katz, S. (1989). Risk of functional decline among well elders. Journal of Clinical Epidemiology, 42, 895-904.

Morey, M.C., Pieper, C.G., Crowley, G.M., Sullivan, R.J., \& Puglisi, C.M. (2002). Exercise adherence and 10-year mortality in chronically ill older adults. Journal of the American Geriatrics Society, 50, 1929-1933.

Morris, M. (2000). Movement disorders in people with Parkinson’s disease: A model for physical therapy. Physical Therapy, 80, 578-597.

Morris, M.E., Iansek, R., Matyas, T.A., \& Summers, J.J. (1994). The pathogenesis of gait hypokinesia in Parkinson's disease. Brain, 117(5), 1169-1181.

Morris, M.E., McGinley, J., Huxham, F., Collier, J., \& Iansek, R. (1999). Constraints on the kinetic, kinematic and spatiotemporal parameters of gait in Parkinson's disease. Human Movement Science, 18, 461-483. 
Murtch, W.J., Strudwick, A., Roy, S.K., \& Downie, A.W. (1986). Parkinson’s disease: Disability, review and management. British Medical Journal, 293(13), 675-677.

Muslimovic, D., Post, B., Speelman, J.D., Schmand, B., deHaan, R.J., \& CARPA study group (2008). Determinants of disability and quality of life in mild to moderate Parkinson's disease. Neurology, 70(23), 2241-2247.

Nallegowda, M., Singh, U., Handa, G., Khanna, M., Meeka, S., Yadav, S., Kumar, G., \& Behari, M. (2004). Role of sensory input and muscle strength in maintenance of balance, gait and posture in Parkinson's disease: A pilot study. American Journal of Medical and Physical Rehabilitation, 83(12), 898-908.

Nocera, J.R., Altmann, L.J., Sapienza, C., Okum, M.S., \& Hass, C.J. (2010). Can exercise improve language and cognition in Parkinson's disease? Neurocase. 16, 301-306.

Nogaki, H., Fukusako, T., Sasabe, F., Negoro, K., \& Morimatsu, M. (1995). Muscle strength in early Parkinson’s disease. Movement Disorders, 10, 225-226.

Nogaki, H., Kakinuma, S., \& Morimatsu, M. (1999). Movement velocity dependent muscle strength in Parkinson’s disease. Acta Neurologica Scandinavica, 99(3), 152-157.

Nordander, C., Ohlsson, K., Balogh, I., Hansson, G.A., Axmon, A., Persson, R., \& Skerfving, S. (2008). Gender differences in workers with identical repetitive industrial tasks: Exposure and musculoskeletal disorders. Internal Archives of Occupational and Environmental Health, 81, 939-947.

Obeso, J.A., Rodriguez-Oroz, M., Rodriguez, M., Lanciego, J.L., Artieda, J., Gonzalo, N., \& Olanow, C.W. (2000). Pathophysiology of the basal ganglia in Parkinson's disease. Trends Neuroscience, 23, S8-S19

Olanow, C.W. \& McNaught, K. (2011). Parkinson’s disease, proteins, and prions: Milestones. Movement Disorders, 26(6), 1056-1071.

Olanow, C.W., Brin, M. \& Obeso, J.A. (2000). The role of deep brain stimulation as surgical treatment for Parkinson's disease. Neurology, 55(S6), 60-66.

Pääsuke, M., Ereline, J., Gapeyeva, H., Joost, K., Mottus, K., \& Taba, P. (2004). Leg-extension strength and chair-rise performance in elderly women with Parkinson's disease. Journal of Aging and Physical Activity, 12(4), 511-524.

Pääsuke, M., Mottus, K., Ereline, J., Gapeyeva, H., \& Taba, P. (2002). Lower limb performance in older female patients with Parkinson's disease. Aging Clinical and Experimental Research, 14(3), 185-191.

Paganelli, R., Di Iorio, A., Cherubini, A., Lauretani, F., Mussi, C., Volpato, S. ... Ferrucci, L. (2006). Frailty of older age: The role of the endocrine--immune interaction. Current Pharmaceutical Des, 12(24), 3147-3159.

Pavon, J.M., Whitson, H.E., \& Okun, M.S. (2010). Parkinson's disease in women: A call for improved clinical studies and for comparative effectiveness research. Maturitas, 65, 352358.

Pedersen, S.W., Oberg, B., Larsson, L., \& Lindval, B. (1997). Gait analysis, isokinetic muscle strength measurement in patients with Parkinson's disease. Scandinavica Journal of Rehabilitation Medicine, 29(2), 67-74. 
Pel-Little, R.E., Schuurmans, M.J., Emmelot-Vonk, M.H., \& Verhaar, H.J. (2009). Frailty: Defining and measuring of a concept. Journal of Nutrition, Health and Aging, 13, 390394.

Pfann, K.D., Buchman, A.S., Comella, C.L., \& Corcos, D.M. (2001). Control of movement distance in Parkinson's disease. Movement Disorders, 16(6), 1048-1065.

Poehlman, E.T., Toth, M.J., Fishman, P.S., Vaikevicius, P., Gottlieb, S.S., Fisher, M.L. \& Fonong, T. (1995). Sarcopenia in aging humans: The impact of menopause and disease. Journal of Gerontology A Biological Sciences Medical Sciences, 50, 73-78.

Pohjalainen, T., Rinne, J. O., Nagren, K., Syvalahti, E., \& Hietala, J. (1998). Sex differences in the striatal dopamine D2 receptor binding characteristics in vivo. American Journal of Psychiatry, 155, 768-773.

Powell, C. (1997). Frailty: help or hindrance? Journal of the Royal Society of Medicine, 90(S32), 23-26.

Powell, C. (2008). Frailty and Parkinson's disease: Theories and clinical implications. Parkinsonism and Related Disorders, 4(3), 271-272.

Protas, E.J., Stanley, R.K., \& Jankovic, J. (1996). Exercise and Parkinson's disease. Critical Reviews in Physical Rehabilitation Medicine, 8(4), 253-266.

Puts, M.T., Lips, P., \& Deeg, D.J. (2005). Sex differences in the risk of frailty for mortality independent of disability and chronic diseases. Journal of the American Geriatrics Society, 53(1), 40-47.

Quinn, L., Busse, M., Khalil, H., Richardson, S., Rosser, A., \& Morris, H. (2009). Client and therapist views on exercise programmes for early-mid stage Parkinson's disease and Huntington's disease. Disability and Rehabilitation, Early Online, 1-12.

Rahman, S., Griffin, H.K., Quinn, N.P., \& Jahanshahi, M. (2008). Quality of life in Parkinson's disease: The relative importance of symptoms. Movement Disorders, 23(10), 1428-1434.

Ramaker, C., Marinus, J., Stiggelbout, A.M., \& van Hilten, B.J. (2002). Systemic evaluation of rating scales for impairment and disability in Parkinson's disease. Movement Disorders, 17, 867-876.

Reichmann, H. \& Emre, M. (2012). Optimizing levodopa therapy to treat wearing-off symptoms in Parkinson's disease: Focus on levodopa/carbidopa/entacapone. Expert Reviews of Neurotherapeutics, 12(2), 119-131.

Rimmer, J.H. (2005). Exercise and physical activity in persons aging with a physical disability. Physical Medicine Rehabilitation Clinical North American, 16, 41-56.

Robichaud, J.A., Pfann, K.D., Comella, C.L., \& Corcos, D.M. (2002). Effect of medication on EMG patterns in individuals with Parkinson's disease. Movement Disorders, 17(5), 950960.

Robichaud, J.A., Pfann, K.D., Vaillancourt, D.E., Comella, C.L., \& Corcos, D.M. (2005). Force control and disease severity in Parkinson's disease. Movement Disorders, 20(4), 441-450.

Rockwood, K. \& Mitnitski, A. (2007). Frailty in relation to the accumulation of deficits. Journals of Gerontology A Biological Sciences Medical Sciences, 62(7), 722-727.

Rockwood, K., Fox, R.A., Stolee, P., Robertson, D., \& Beattie, B.L. (1994). Frailty in elderly people; An evolving concept. Canadian Medical Association Journal, 150, 489-495. 
Roland, K.P., Theou, O., Jakobi, J.M., Swan, L., \& Jones, G. R. (2011a). Exploring frailty: Community physical and occupational therapists' perspectives. Physical and Occupational Therapy in Geriatrics, 29(4), 270-286.

Roland, K.P., Jakobi, J.M., Powell, C., \& Jones, G. R. (2011b). Factors related to functional independence in females with Parkinson's disease: A systematic review. Maturitas, 69(4), 304-311.

Roland, K.P., Jakobi, J.M., Powell, C., \& Jones, G.R. (2012). Quality of life as a determinant of frailty phenotype in community-dwelling persons with Parkinson's disease. Journal of the American Geriatrics Society, 60(3), 590-592.

Roland, K.P., Cornett, K.M.D., Theou, O., Jakobi, J.M., \& Jones, G.R. (Accepted). Daily physical activity across frailty phenotypes in community-dwelling females with and without Parkinson’s disease. The Journal of Aging Research (\#468156).

Rothman, M.D., Leo-Summers, L., \& Gill, T.M. (2008). Prognostic significance of potential frailty criteria. Journal of the American Geriatrics Society, 56, 2211-2216.

Rubin, S. M. (2007). Parkinson’s disease in women. Disease-a-Month, 53, 206-213.

Salenius, S., Vikainen, A., Kaakkola, S., Hari, R., \& Brown, P. (2002). Defective cortical drive to muscle in Parkinson's disease and its improvement with levodopa. Brain, 125(3), 491500 .

Schapira, A.H.V. (2006). The etiology of Parkinson's disease. Neurology, 66(4), S10-S23.

Schenkman, M., Hall, D., Kumar, R., \& Kohrt, W.M. (2008). Endurance exercise training to improve economy of movement of people with Parkinson disease: Three case reports. Physical Therapy, 88(1), 63-76.

Schenkman, M., Morey, M., \& Kuchibhatla, M. (2000). Spinal flexibility and balance control among community-dwelling adults with and without Parkinson's disease. Journal of Gerontology Series A Biological Sciences, 55(8), M441-445.

Schrag, A. (2000). What contributes to quality of life in patients with Parkinson's disease. Journal of Neurology, Neurosurgery and Psychiatry, 69(3), 308.

Schrag, A., Jahanshahi, M., \& Quinn, N. (2000). How does Parkinson's disease affect quality of life? A comparison with quality of life in the general population. Movement Disorders, 15(6), 1112-1118.

Scott, B., Borgman, A., Engler, H., Johnels, B., \& Aquilonius, S.M. (2000). Gender differences in Parkinson's disease symptom proile. Acta Neurologica Scandica, 102, 37-43.

Seeman, T.E., Berkman, L.F., Charpentier, P.A., Blazer, D.G., Albert, M.S., \& Tinetti, M.E. (1995). Behavioral and psychosocial predictors of physical performance: MacArthur studies of successful aging. Journal of Gerontology A Biological Sciences Medical Sciences, 50, M177-M180.

Shardell, M., Hicks, G.E., Miller, R.R., Kritchevsky, S., Andersen, D., Bandinelli, S. ... Ferrucci, L. (2009). Association of low vitamin D levels with the frailty syndrome in men and women. Journals of Gerontology A Biological Science Medical Science, 64(1), 69-75.

Shaw, C. Parkinson's disease exercise? Why not! Vancouver Coastal Health; catalogue \# FM.490.P229. 
Shore, W.S. \& DeLateur, B.J. (2007). Prevention and treatment of frailty in the postmenopausal woman. Physical Medicine Rehabilitation Clinic North America, 18(3), 609-621.

Shulman, L.M. (2007). Gender differences in Parkinson’s disease. Gender Medicine, 4(1), 8-18.

Smith, J. \& Baltes, M.M. (1999). The role of gender in very old age: Functioning and everyday life patterns. Psychological Aging, 13(4), 676-695.

Smith, M.C., Ellgring, H., \& Oertel, W.H. (1995). Sleep disturbances in Parkinson’s disease patients and spouses. Journal of the American Geriatrics Society, 45(2), 194-199.

Solimeo, S. (2008). Sex and Gender in Older Adults' Experience of Parkinson's disease. Journal of Gerontology, 63B(1), S42-S48.

Speelman, A.D., van de Warrenburg, B.P., van Nimwegen, M., Petzinger, G.M., Munneke, M., \& Bloem, B. (2011). How might physical activity benefit patients with Parkinson's disease. Nature Review Neurology, 7, 528-534.

Sri Cherukmilli, V. \& Kavanaugh, A. (2011). Early onset rheumatoid arthritis. Geriatric Rheumatology, 3, 145-150.

Stanley, R.K., Protas, E.J., \& Jankovic, J. (1999). Exercise performance in those having Parkinson's disease and healthy normals. Medical Science in Sports and Exercise, 31(6), 761-766.

Stelmach, G.E., Teasdale, N., Phillips, J., \& Worringham, C.J. (1989). Force production characteristics in Parkinson’s disease. Experimental Brain Research, 76, 165-171.

Sutoo, D. \& Akiyama, K. (2003). Regulation of brain function by exercise. Neurobiological Discovery, 13, 1-14.

Syddall, H., Cooper, C., Martin, F., Briggs, R., \& Aihie Sayer, A. (2003). Is grip strength a useful single marker of frailty? Age and Ageing, 32(6), 650-656.

Taggart, H. \& Crawford, V. (1995). Reduced bone density of the hip in elderly patients with Parkinson's disease. Age and Ageing, 24, 326-328.

Taylor, H.L., Jacobs, D.R., Schucker, B., Knudsen, J., Leon, A.S., \& Debacker, G. (1978). A questionnaire for the assessment of leisure time physical activities. Journal of Chronic Disease, 31(12), 741-755.

Teasdale, N., Phillips, J., \& Stelmach, G.E. (1990). Temporal movement control in patients with Parkinson’s disease. Journal of Neurology Neurosurgery and Psychiatry, 53, 862-868.

Theou, O., Jones, G.R., Vandervoort, A.A., \& Jakobi, J.M. (2010). Daily muscle activity and quiescence in non-frail, pre-frail, and frail older women. Experimental Gerontology, 45, 909-917.

Theou O., Bruce S., Roland K.P., Jones G.R., \& Jakobi J.M. (2011a). Long-term electromyography recordings in community- and retirement-dwelling older women. Gerontechnology, 10(3), 136-131.

Theou, O., Stathokostas, L., Roland, K.P., Jakobi, J.M., Patterson, C., Vandervoort, A.A., \& Jones G.R. (2011b). The effectiveness of exercise interventions for the management of frailty: A systematic review. Journal of Aging Research, \#569194.

Toth, M.J., Fishman, P.S., \& Poehlman, E.T. (1997). Free-living daily energy expenditure in patients with Parkinson's disease. Neurology, 48(1), 88. 
Tsang, K.L., Jiang, H., Ramsden, D.B., \& Ho, S.L. (2001). The use of estrogen in the treatment of Parkinson's disease. Parkinsonism and Related Disorders, 8, 133-137.

Tsang, K., Ho, S., \& Lo, S. (2000). Estrogen improves motor disability in Parkinsonian postmenopausal women with motor fluctuations. Neurology, 54(12), 2292-2298.

Twelves, D., Perkins, K.S.M., \& Counsell, C. (2003). Systematic review of incidence studies in Parkinson's disease. Movement Disorders, 18(1), 19-31.

Vandervoort, A.A. (2002). Aging of the human neuromuscular system. Muscle and Nerve, 25(1), 17-25.

Venglar M. (2005). Case report: Tai chi and parkinsonism. Physiotherapy Research International, 10(2), 116-121.

Visser, M., Marinus, J., van Hilten, J.J., Schipper, R.G.B., \& Stiggelbout, A.M. (2004). Assessing comorbidity in patients with Parkinson's disease. Movement Disorders, 19(7), 824-838.

Voznesensky, M., Walsh, S., Dauser, D., Brindisi, J., \& Kenny, A.M. (2009). The association between dehydroepiandosterone and frailty in older men and women. Age and Ageing, 38(4), 401-406.

Webber, S.C. \& Porter, M.M. (2009). Monitoring mobility in older adults using global positioning system (GPS) watches and accelerometers: A feasibility study. Journal of Aging and Physical Activity, 17(7), 455-467.

Westerterp, K.R. (2009). Assessment of physical activity: A critical appraisal. European Journal of Applied Physiology, 105(6), 823-828.

Whiston, H.E., Purser, J.L., \& Cohen, H.L. (2007). Frailty thy name is... phrailty? Journal of Gerontology A Biological Science Medical Science, 62(7), 728-730.

Wierzbicka, M.M., Wiegner, A.W., Logigian, E.L., \& Young, R.R. (1991). Abnormal mostrapid isometric contractions in patients with Parkinson's disease. Journal of Neurology, Neurosurgery and Psychiatry, 54(3), 210-216.

Wolf, S.L., Barnhart, H.X., Ellison, G.L., Coogler, C.E., \& Atlanta FICSIT Group. (1997). The effect of tai chi quan and computerized balance training on postural stability in older subjects. Physical Therapy, 77(4), 371-381

Wong, C.H., Weiss, D., Sourial, N., Karunananthan, S., Quail, J.M., Wolfson, C., \& Bergman, H. (2010). Frailty and its association with disability and comorbidity in a communitydwelling sample of seniors in Montreal: A cross-sectional study. Aging, Clinical and Experimental Research, 22(1), 54-62.

Woo, J., Goggins, W., Sham, A., \& Ho, S.C. (2005). Social determinants of frailty. Gerontology, 51(6), 402-408.

Xue, Q., Fried, L.P., Glass, T.A., Laffan, A., \& Chaves, P.H.M. (2008). Life-space constriction, development of frailty, and the competing risk of mortality. American Journal of Epidemiology, 167(2), 240-248. 


\title{
Appendices
}

\section{Appendix A: Consent Form}

\author{
Assessing Sex- and Stage-differences in Muscle Activity Among Individuals Living with Parkinson's disease
}

\section{Principal Investigator:}

Dr. Gareth R. Jones, PhD, Assistant Professor Human Kinetics, UBC Okanagan.

Ph:xxx.xxx.xxxx; Email: xxxx@ubc.ca

Co-Investigator:

Dr. Jennifer Jakobi, PhD, Assistant Professor Human Kinetics, UBC Okanagan.

Ph:xxx.xxx.xxxx; Email: xxxx@ubc.ca

Kaitlyn P. Roland, PhD (student) Human Kinetics, UBC Okanagan.

Ph:xxx.xxx.xxxx; Email:xxxx@ubc.ca

You are being invited to participate in a research study looking at muscle activity in persons living with Parkinson's disease. You must be over 50 years of age are with or without Parkinson's disease. The purpose of this letter is to provide you with the information you need to make an informed decision about participating in this research.

\section{Voluntary participation and other pertinent information}

Your participation in this study is completely voluntary, and it is up to you whether or not you choose to partake in this study. Should you choose to participate, you will be required to sign the consent form at the end of this booklet. However, you are free to withdraw from this study at any point in time if you wish to discontinue your participation without providing any reasons.

This booklet will provide you with all necessary information regarding the study, so please review it with care and discuss it with your family, friends and doctor before you decide if you are going to participate or not.

If you choose not to participate in this study, you will not be penalized in any way, nor do you need to disclose why you have chosen not to participate.

You have been given all the important information regarding your participation in this study. You should only agree to participate if you feel happy that you know enough about these things. If you are participating in another study at this time, please inform the lead study investigator to determine if your participation in this study is appropriate.

This study is being conducted at the Human Kinetics Department of the University of British Columbia Okanagan, Kelowna B.C. with the help of internal funding from the Faculty of Health and Social Development. Please request any details concerning this compensation to the Principal Investigator, Dr. Gareth R. Jones. 


\section{Background Information}

Portable electromyography (EMG) can examine muscle activity during real life movement. This investigation will use EMG to measure muscle activity in persons with Parkinson's disease over the course of a full 8-hour day where factors such as fatigue and environmental stress may impact muscle activity. Portable EMG records electrical activity produced by muscles via surface electrodes. Unlike traditional EMG, the portable unit is worn on a waist belt and does not restrict measurement to lab-based tasks. This will enable us to define muscle activity that is used to execute movement and determine the practicality and reliability of using EMG as a clinical assessment tool.

\section{Purpose of this study}

This research is being done to explore sex- and stage-differences in muscle activity in persons living with Parkinson's disease compared to those without. The aim is to determine muscle activity through EMG characteristics in men and women at different stages of Parkinson's disease. The goal is to provide further resources to help diagnose and treat Parkinson's disease in community.

\section{Who can participate?}

If you are a male or female adult living with Parkinson's disease, you are welcome to participate in this study. Also, if you are a healthy male or female adult, ages 50 and older you are welcome to participate. You will be screened for the ability to walk and move around independently (using a walk-aid as necessary), any other severe cognitive impairment or other severe neurological disorders. Also, those with a Hoehn-Yahr score greater that 4 will be excluded because of the severity of the Parkinson's disease and high likelihood of disability. You must be able to speak and read English fluently.

\section{Who should not participate?}

You should not participate in this study if you are: (1) Unable to ambulate independently, even with the help of a walk-aid; (2) You have severe cognitive impairment or (3) a neurological disorder other than Parkinson's disease; (4) You have a Hoehn-Yahr score greater than 4; and (5) You are unable to read or speak English fluently.

\section{Procedure for this study}

Should you choose to participate in this study, your participation would involve completing questionnaires (health, physical activity, quality of life) a walking and balance test, then wearing a portable electromyography device during the course of an 8-hour day, which will have surface electrodes attached to the two muscles of your arms (biceps, triceps) and two muscles of your legs (quadriceps, hamstrings). This is a one-time assessment. 
1. The research assistant will visit you in your home in the early morning (7-10 am) to do a health history and physical activity questionnaire. You do not have to answer any questions that you are uncomfortable answering.

2. The research assistant will measure: time it takes to walk 15-ft, grip strength (dynamometer), balance (Berg Balance Scale) and gait (Dynamic Gait Index).

3. The research assistant will install a portable electromyography (EMG) unit, the size of a small tape recorder to measure your upper and lower body muscle activity. EMG surface electrodes (small square band-aids) will be placed over your skin over arm and leg muscles and covered in mesh to assure adherence. Three maximal contractions (hard as you can) per muscle will be performed to ensure the device is working properly.

3. Your physical activity will be monitored using; a waist -born accelerometer (size of a matchbook) to measure lower movement accelerations per minute; a heart rate monitor (chest strap); and a wrist-born (watch) global positioning system (GPS) will monitor gross movement per minute. Total set-up will take approximately one hour.

4. You will wear this equipment for 8 hours, during normal waking hours and continue with your normal daily activities.

5. After the day-long assessment the electrodes will be removed by the research assistant at your residence. This will take no longer than 30 minutes.

The study will involve a total of 20 men and 20 women living with Parkinson's disease (stage 12, 3), and 20 men and 20 women, age-matched, to serve as controls.

\section{Your responsibilities}

It is important that you continue with your normal daily activities and medication schedule for the duration of the testing. However, you will be asked to refrain from any water-based activities while you are wearing the portable EMG (i.e. baths, swimming).

\section{Risks and discomforts of participation}

The risks associated with the proposed research are minimal. In individuals with allergies to 'band-aid'-like adhesives, minimal skin reactions to adhesive tape may occur. Minimal muscle soreness may result from maximal contractions. The activity monitors will not impede movements and participation in typical activities of daily living will not be impeded. We ask you not to get the devices wet (i.e. no baths, showers or swims).

\section{Associated benefits of participation}

There are no direct benefits to you, except the results of an assessment of your daily muscle activity, physical activity (step count and calories), heart rate, balance and gait.

Changes to muscle activity may perpetuate muscle weakness and increased fall-risk, making it a predictor of functional decline. By targeting persons living with Parkinson's disease, we could provide significant information regarding physiological changes that lead to stages of functional decline in women and men living with Parkinson's disease. 


\section{Will I be paid or do I have to pay to participate?}

You will not be paid for participating and there is no cost to participating in the described experiments. However, any incurred parking expenses as a result of your participation in this study will be reimbursed, provided receipts are submitted to a member of the research team. You are free to withdraw from the experiment at any point in time. You do not have to provide a reason for withdrawing from the study if you do not wish to do so.

\section{What to do if you want to withdraw from this study:}

Participation in this study is voluntary. You have the right to refuse to participate, refuse to answer any questions or withdraw from the study at any time with no effect on your future (care/academic status/employment). You do not have to provide any reasons to do so. If you choose to enter the study and then decide to withdraw at a later time, all data collected about you during your enrolment in the study will be retained for analysis. By law, this data cannot be destroyed.

\section{What happens if something goes wrong during the study?}

Any adverse event that should arise will be followed-up thoroughly to ensure your safety and health. Background health history and functional assessment parameters will be collected and stored on a computer, while any clinical symptoms will be recorded in the Investigator's laboratory book. All data arising from this study will be archived and stored securely by the Investigators as described above, and remain confidential. You do not waive any legal rights by signing the consent form.

The researchers of this study will be readily available if you would like to discuss any problems or concerns that may arise. Following completion of the project you will be provided with a feedback sheet explaining the outcomes and any substantive findings.

\section{Can I be asked to leave the study?}

If you do not adhere to the study guidelines outlined earlier in this study, you will be asked to leave the study. Also, in the rare event that a medical emergency occurs during the study, you will be automatically withdrawn from the study to ensure your safety and well-being.

\section{After this study is complete:}

Results of this project may be published, but any data included will not be linked to a specific participant. Your data will be assigned a personal identification number to ensure anonymity in both the analysis and documentation of results. The raw data obtained in this study will only be available to the principle investigator (Dr Jones). As stated earlier, you will be provided with a feedback sheet explaining the outcomes and specific findings of this study.

\section{What will the study cost me?}

You will not incur any personal expenses as a result of participation. You will not be paid for participating. 


\section{Privacy and confidentiality}

Your confidentiality will be respected. No information that discloses your identity will be released or published without your explicit consent to the disclosure. However, research records and medical records identifying you may be inspected in the presence of the Investigator or his or her designate by representatives the UBC Clinical Research Ethics Board for the purpose of monitoring the research. However, no records which identify you by name or initials will be allowed to leave the Investigators' offices.

Personal descriptors (i.e. names) will be coded to a numeric value and data will be kept in a locked cabinet in Fine Arts 251 at the University of British Columbia Okanagan. The data will be made available only to members of the research team, and destroyed in 5 years. The master copy will be stored separate from the coded data.

If you have any concerns about your rights as a research subject and/or your experiences while participating in this study, contact the Research Subject Information Line in the University of British Columbia Office of Research Services by email at RSIL@ors.ubc.ca or by phone xxx$\mathrm{xxX}-\mathrm{XXXx}$. 


\section{CONSENT FORM FOR PARTICIPANTS}

I have read and understand the information sheet concerning this project. All my questions have been answered to my satisfaction. I understand that I am free to request further information at any stage. I know that:

1. My participation in the project is entirely voluntary, and I am free to withdraw from this study at any time without any disadvantage.

2. The data on which the results of the project depend will be retained in secure storage for five years, after which they will be destroyed.

3. I will be required to complete initial assessments and then one-day wearing the portable equipment, where I will continue with my normal daily activities.

4. Each experimental session will involve the following measurements:

- demographic \& medical history questionnaires, basic physiological measures, Hoehn and Yahr stage of disease, Berg Balance Scale, frailty phenotype, Dynamic Gait Index, and Parkinson's disease Questionnaire-39.

- Assessment of 8-hour daily muscle activity using portable EMG.

5. The results of the project may be published and will be available in the University of British Columbia Okanagan Library but every attempt will be made to preserve my anonymity.

6. I will receive a signed and dated copy of this consent form.

I agree to take part in this project

Printed name of Subject

Signature

Date

Printed name of principal investigator

Signature

Date

Project has been reviewed \& approved by UBC Clinical Research Ethics Board. This Board aims to help protect the rights of research subjects. 


\section{Appendix B: Health History Questionnaire}

Note: questionnaire will be destroyed if subject does not qualify to participate.

Only study codes, which do not identify you the subject directly, will be used.

Date of Experiment: Experimenter Name(s):

Subject Name:

Subject Code *study codes only will be used

Sex: M / F Date of Birth (mm/yyyy):

Weight (kg):_Height $(\mathrm{cm})$ :

Dominant Hand: $\quad$ Right Left

Mailing Address (For Experiment Findings Only):

Phone Number (and email):

1. Are you a regular smoker?

YES

$\square$ NO

If yes, how often?

2. Have you had surgery in the past year? $\square$ YES $\square$ NO

If yes, what type?

3. Have you been diagnosed by a health professional as having any of the following? (Check all that apply, and be specific where applicable)

Heart Trouble

Arthritis

High Blood Pressure

High Cholesterol

Cardiac Pacemaker

Electronic Implant

Back problems

Foot problems

Muscle problems

Bone or Joint disorder

Previous Injury

Alcoholism

Diabetes 
4. Do you suffer from any allergies? (Include hay fever and sinus problems)

5. Do you have difficulty hearing?

6. Do you have difficult seeing?

7. Other (than PD) health problems?

8. PD duration:

years

9. PD side most affected:

10. Are you currently using any medications? (include dosage and time)

11. Do you use any gait-aids? (ie: walker, cane)

YES

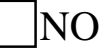

If yes, how often and for what purposes? (ie: long distances, outside travel etc.)

12. In the last year, have you lost more than 10 pounds unintentionally (i.e., not due to dieting or exercise)?

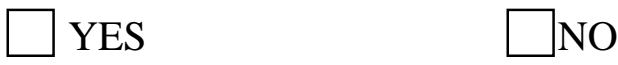

13. How often in the last week did you feel that everything you did was an effort?

rarely or none of the time ( $<1$ day)

some or a little of the time (1-2 days)

$\square$ moderate amount of the time (3-4 days)

$\square$ most of the time

14. How often in the last week did you feel that you could not get going?

$\square$ rarely or none of the time ( $<1$ day)

some or a little of the time (1-2 days)

$\square$ moderate amount of the time (3-4 days) $\square$ most of the time 
Appendix C: Minnesota Leisure Time Physical Activity Questionnaire (MLTA)

\begin{tabular}{|c|c|c|c|c|c|}
\hline \multirow[t]{2}{*}{ ACTIVITY } & \multicolumn{2}{|c|}{$\begin{array}{c}\text { Did you } \\
\text { perform this } \\
\text { activity the last } \\
2 \text { weeks? }\end{array}$} & \multirow[t]{2}{*}{$\begin{array}{l}\text { How many times } \\
\text { did you do this } \\
\text { activity the last } 2 \\
\text { weeks? }\end{array}$} & \multicolumn{2}{|c|}{$\begin{array}{l}\text { How long did } \\
\text { you usually do } \\
\text { the activity } \\
\text { each time? }\end{array}$} \\
\hline & NO & YES & & Hrs & Min \\
\hline Walking for exercise & & & & & \\
\hline Moderate strenuous house & & & & & \\
\hline Mowing the lawn & & & & & \\
\hline Raking the lawn & & & & & \\
\hline Gardening & & & & & \\
\hline Hiking & & & & & \\
\hline Jogging & & & & & \\
\hline Biking & & & & & \\
\hline Exercise Cycle & & & & & \\
\hline Dancing & & & & & \\
\hline Aerobics & & & & & \\
\hline Bowling & & & & & \\
\hline Golf & & & & & \\
\hline Single Tennis & & & & & \\
\hline Doubles Tennis & & & & & \\
\hline Racquetball & & & & & \\
\hline Calisthenics/Weights & & & & & \\
\hline Swimming & & & & & \\
\hline
\end{tabular}




\section{Appendix D: Parkinson's Disease Questionnaire-39}

Subject:

date:

Please check that you have ticked one box for each question before going to the next.

Due to having Parkinson's disease, how often during the last month have you....

\begin{tabular}{|c|c|c|c|c|c|}
\hline & Never & Occasionally & Sometimes & Often & Always \\
\hline $\begin{array}{l}1 \text { Had difficulty doing the leisure activit } \\
\text { which you would like to do? }\end{array}$ & & & & & \\
\hline $\begin{array}{l}2 \text { Had difficulty looking after your home } \\
\text { e.g. DIY, housework, cooking? }\end{array}$ & & & & & \\
\hline $\begin{array}{l}3 \text { Had difficulty carrying bags of } \\
\text { shopping? }\end{array}$ & & & & & \\
\hline 4 Had problems walking half a mile? & & & & & \\
\hline 5 Had problems walking 100 yards? & & & & & \\
\hline $\begin{array}{l}6 \text { Had problems getting around the hol } \\
\text { as easily as you would like? }\end{array}$ & & & & & \\
\hline 7 Had difficulty getting around in public & & & & & \\
\hline $\begin{array}{l}8 \text { Needed someone else to accompany } \\
\text { you when you out? }\end{array}$ & & & & & \\
\hline $\begin{array}{l}9 \text { Felt frightened or worried about fallin } \\
\text { over in public? }\end{array}$ & & & & & \\
\hline $\begin{array}{l}10 \text { Been confined to the house more th } \\
\text { you would like? }\end{array}$ & & & & & \\
\hline 11 Had difficulty washing yourself? & & & & & \\
\hline 12 Had difficulty dressing yourself? & & & & & \\
\hline $\begin{array}{l}13 \text { Had problems doing up your } \\
\text { shoelaces? }\end{array}$ & & & & & \\
\hline 14 Had problems writing clearly? & & & & & \\
\hline 15 Had difficulty cutting up your food? & & & & & \\
\hline
\end{tabular}




\begin{tabular}{|c|c|c|c|c|c|}
\hline & Never & Occasionally & Sometimes & Often & Always \\
\hline $\begin{array}{l}16 \text { Had difficulty holding a drink without } \\
\text { spilling it? }\end{array}$ & & & & & \\
\hline 17 Felt depressed? & & & & & \\
\hline 18 Felt isolated and lonely? & & & & & \\
\hline 19 Felt weepy or tearful? & & & & & \\
\hline 20 Felt angry or bitter? & & & & & \\
\hline 21 Felt anxious? & & & & & \\
\hline 22 Felt worried about your future? & & & & & \\
\hline $\begin{array}{l}23 \text { Felt you had to conceal your } \\
\text { Parkinson's from people? }\end{array}$ & & & & & \\
\hline $\begin{array}{l}24 \text { Avoided situations like eating or } \\
\text { drinking in public? }\end{array}$ & & & & & \\
\hline $\begin{array}{l}25 \text { Felt embarrassed in public due to } \\
\text { having Parkinson's disease? }\end{array}$ & & & & & \\
\hline $\begin{array}{l}26 \text { Felt worried by other people's reaction } \\
\text { to you? }\end{array}$ & & & & & \\
\hline $\begin{array}{l}27 \text { Had problems with your close personal } \\
\text { relationships? }\end{array}$ & & & & & \\
\hline $\begin{array}{l}28 \text { Lacked support in the ways you need } \\
\text { from your spouse or partner? }\end{array}$ & & & & & \\
\hline $\begin{array}{l}29 \text { Lacked support in the ways you need } \\
\text { from your family or friends? }\end{array}$ & & & & & \\
\hline $\begin{array}{l}30 \text { Unexpectedly fallen asleep during the } \\
\text { day? }\end{array}$ & & & & & \\
\hline $\begin{array}{l}31 \text { Had problems with your concentration, } \\
\text { e.g. when reading or watching TV? }\end{array}$ & & & & & \\
\hline 32 Felt your memory was bad? & & & & & \\
\hline $33 \mathrm{Had}$ distressing dream/ hallucinations? & & & & & \\
\hline
\end{tabular}




\begin{tabular}{|l|l|l|l|l|l|}
\hline 34 Had difficulty with your speech? & Never & Occasionally & Sometimes & Often & Always \\
\hline 35 Felt unable to communicate with & & & & & \\
people properly? & & & & & \\
\hline 36 Felt ignored by people? & & & & & \\
\hline 37 Had painful muscle cramps or & & & & & \\
\hline spasms? & & & & & \\
\hline body? & & & & & \\
\hline 39 Felt unpleasantly hot or cold? & & & & & \\
\hline
\end{tabular}

Thank you! 


\section{Appendix E: Copyright Permissions for Reproduction of Journal Articles}

\section{Copyright Permission for Chapter 2}

From: Margaret Rees >margaret.rees@obs-gyn.ox.ac.uk>

Subject: $\quad$ RE: Copyright

Date: $\quad 2$ March, 2012 12:24:09 AM PST

To: $\quad$ Kate Roland

Best wishes for your thesis

Margaret

Prof Margaret Rees MA DPhil FRCOG

Editor in Chief Maturitas

Reader Emeritus in Reproductive Medicine, University of Oxford

Visiting Professor, Faculty of Medicine, University of Glasgow and Adjunct Associate Professor in the Department of Obstetrics, Gynecology and Reproductive Sciences at the University of Medicine and Dentistry of New Jersey

Women's Centre, John Radcliffe Hospital, Oxford, OX3 9DU UK

From: contacttheeditor@elsevier.com [contacttheeditor@elsevier.com]

Sent: 01 March 2012 19:34

To: Margaret Rees

Subject: Copyright

First Name Sender: Kaitlyn

Last Name Sender: Roland

Email Address: rolandka@interchange.ubc.ca

Subject: Copyright

Journal ID: 505954

Journal Title: Maturitas

Text of Email:

Hello

I am writing to gain copyright for the use of the article "Roland, K.P., Jakobi, J.M. Powell, C., Jones, G. R., 2011. Factors related to functional independence in females with Parkinson's disease: a systematic review. Maturitas, 69(4), 304-311." for my doctoral dissertation.

Please don't hesitate to contact me with any further questions.

Thank you.

Kaitlyn Roland 


\section{Copyright Permission for Chapter 5}

\section{From: Mostafa Salem <mostafa.salem@hindawi.com>}

Subject: Re: manuscript 468156

Date: 25 June, 2012 2:16:27 AM PDT

To: Kaitlyn Roland <rolandka@interchange.ubc.ca>Dear Dr. Roland,

Thank you for your email. I would like to inform you that since you are an author of the manuscript, you have its copy rights. So, you can use it in your dissertation.

Best regards,

Mostafa

$* * * * * * * * * * * * * * * * * * * * * * * * * * * * * * *$

Mostafa Salem

Editorial Staff

Hindawi Publishing Corporation

http://www.hindawi.com

On Fri, Jun 22, 2012 at 6:50 AM, Kaitlyn Roland

<rolandka@interchange.ubc.ca> wrote:

Hello,

This article has been accepted for publication in the Journal of Aging Research. I am writing to request approval to include this as part of my doctoral dissertation.

I appreciate your speedy response on this. Thank you and please don't hesitate to contact me with any further questions.

Kaitlyn Roland

Kaitlyn Roland

Ph.D. candidate,

School of Health \& Exercise Sciences

University of British Columbia Okanagan

Email: rolandka@interchange.ubc.ca 


\section{Copyright Permission for Chapter 6}

\section{From: $\quad$ Permission Requests - UK < permissionsuk@wiley.com>}

Subject: $\quad$ FW: permission for Journal of the American Geriatrics Society

Date: $\quad 5$ March, 2012 8:40:20 AM PST

To: Kate Roland <rolandka@interchange.ubc.ca>

Dear Kaitlyn,

Thank you for your email request.

Permission is granted for you to use the material requested for your thesis/dissertation subject to the usual acknowledgements and on the understanding that you will reapply for permission if you wish to distribute or publish your thesis/dissertation commercially.

Permission is granted solely for use in conjunction with the thesis, and the article may not be posted online separately.

Any third party material is expressly excluded from this permission. If any material appears within the article with credit to another source, authorisation from that source must be obtained.

Best Wishes

Verity Butler

Permissions Assistant

John Wiley \& Sons Ltd.

From: Kaitlyn Roland [mailto:rolandka@interchange.ubc.ca] Sent: Friday, March 02, 2012 4:45 PM To: Permissions - US Subject: Fwd: permission for Journal of the American Geriatrics Society

Hello

I have an accepted article (letter to the editor, jgs3862) that I would like to include as part of my doctoral dissertation. I am writing to gain permission to do so.

Roland, K.P., Jakobi, J.M., Powell, C., \& Jones, G.R. Quality of Life as a Determinant of Frailty Phenotype in Community-dwelling Persons with Parkinson's disease. J. Amer. Geriatr. Soc. (ID JAGS-1118-L-Sep-11.R1).

Please don't hesitate to contact me with any further questions.

Thank you,

Kaitlyn 


\section{Copyright Permission for Chapter 7}

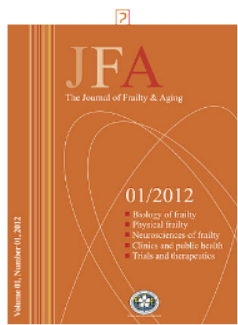

The Journal of Frailty \& Aging

Publisher: Serdi Edition

July 11,2012

To Whom It May Concerns:

This letter is to certify that the manuscript entitled "CONCURRENCE OF FRAILTY AND PARKINSON'S DISEASE" (Manuscript ID \#2012022) by Roland KP, Cornett KMD, Theou O, Jakobi JM, and Jones GR was accepted for publication in the Journal of Frailty \& Aging on June 24,2012 . It is currently scheduled for publication in the next issue (Number 3 ) of the journal that will be released in September 2012.

With the present letter, on behalf of the publisher Serdi Edition, we also authorize the reuse of the article as chapter of the thesis/dissertation by Kaitlyn Roland (first author of the paper), provided that the Journal is acknowledged as the original place of publication.

Sincerely,

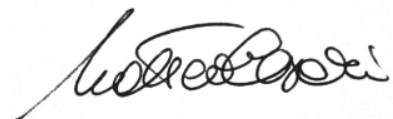

Matteo Cesari, MD, PhD

Editor-in-Chief

The Journal of Frailty \& Aging 